\title{
Extensão dos modelos de memória longa: \\ FI-BREAK e FI-STAR
}

\author{
Victor E. Troster \\ DISSERTAÇÃO APRESENTADA \\ $\mathrm{AO}$ \\ INSTITUTO DE MATEMÁTICA E ESTATÍSTICA \\ DA \\ UNIVERSIDADE DE SÃO PAULO \\ PARA \\ OBTENÇÃO DO TÍTULO DE MESTRE \\ EM \\ CIÊNCIAS \\ Área de Concentração: Estatística \\ Orientadora: Profa. Dra. Clélia Maria de Castro Toloi
}

São Paulo, fevereiro de 2007. 


\title{
Extensão dos modelos de memória longa: FI-BREAK e FI-STAR
}

\author{
Este exemplar corresponde à redação final da \\ dissertação devidamente corrigida e defendida \\ por Victor E. Troster e aprovada \\ pela Comissão Julgadora.
}

São Paulo, 14 de fevereiro de 2007.

Banca Examinadora:

Profa. Dra. Clélia Maria de Castro Toloi (orientadora) - IME/USP

Prof. Dr. Pedro Alberto Morettin - IME/USP

Prof. Dr. Pedro Luis Valls Pereira - IBMEC/SP 


\section{Sumário}

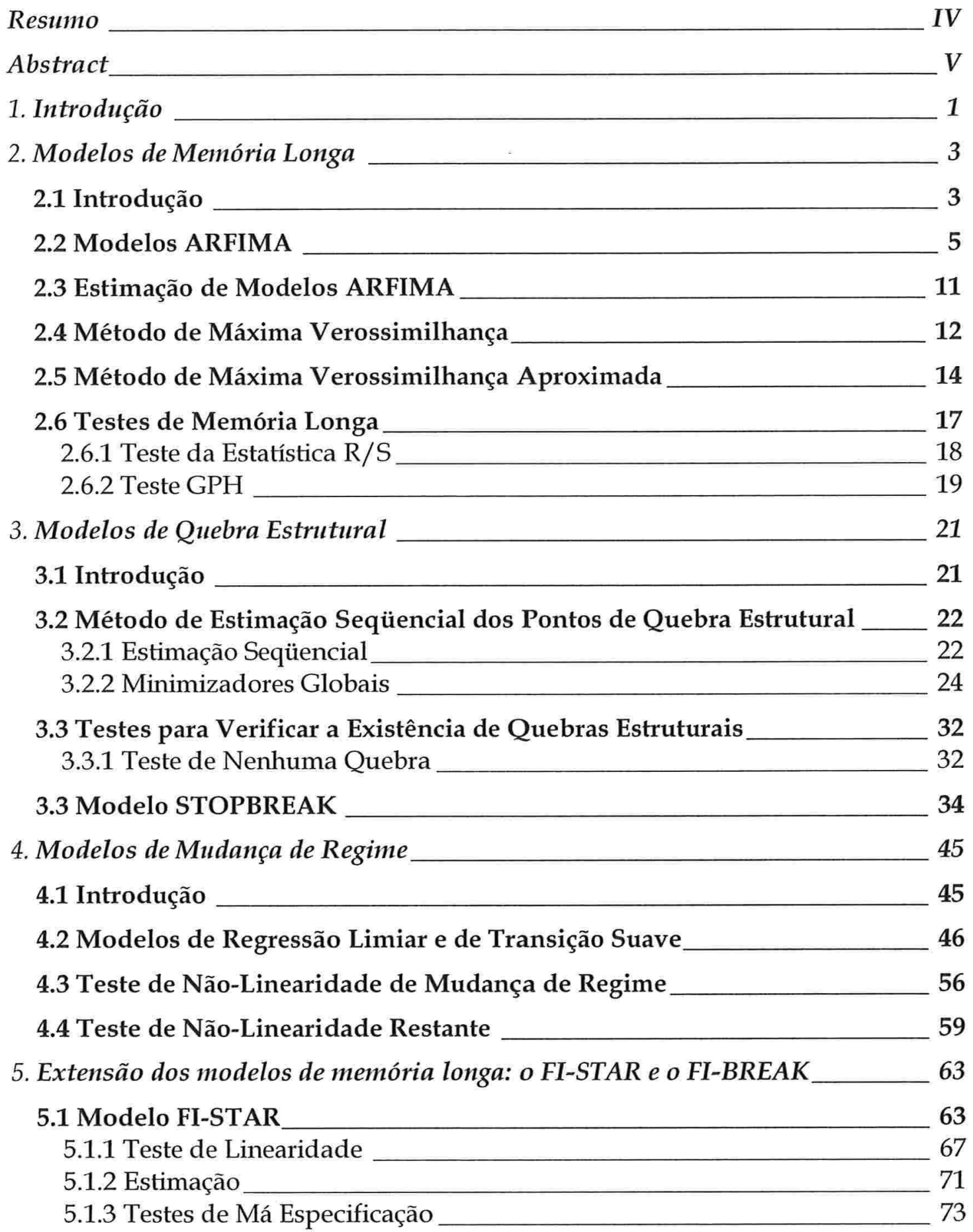


5.2 Modelo FI-BREAK _ 75

5.2.1 Estimação _ 79

6. Aplicação — 81

6.1 Introdução _ 81

6.2 Aplicação do FI-STAR __ 81

6.2.1 Taxa de Desemprego Norte-Americana ___ 81

6.2.2 Retornos Absolutos do IBOVESPA _ 93

6.3 Aplicação do FI-BREAK _ 102

6.3.1 Retornos Mensais da Treasure-Bill (T-Bill) Americana __ 102

6.3.2 Índice de Preços do Trigo de Beveridge __ 110

7. Conclusões — 117

Anexo 119

Referências Bibliográficas__ 125 


\section{Agradecimentos}

Agradeço a Deus, à minha mãe e ao meu pai por ter chegado até aqui.

Gostaria de agradecer muito à professora Clélia Toloi pela excelente orientação que tive para fazer esta dissertação e pelos cursos oferecidos no Mestrado. Sou grato também aos professores Pedro Morettin, Elisabeth Kira e Denise Botter pelos cursos ministrados no Mestrado.

Agradeço ao Marcelo Rabbat pelo incentivo e pelo apoio que me deu para fazer esse curso.

Agradeço também aos meus colegas Marcelo e Lane Rocha, que me ajudaram a escrever os softwares e me ensinaram muito de programação, ao Peterson e ao Marcos que me deram suporte na parte computacional.

Agradeço aos meus amigos Michel, Raul, Rodrigo, Carol, Rafael Terra, Renatão, Israel, Érika, Carolina, Tomás, Tati, Elaine, Marcel, tio Duardo, tia Patsy, vovó Leonor e demais parentes, pela amizade e companheirismo.

Por fim, gostaria de agradecer aos membros da banca. 


\section{Resumo}

Muitas séries temporais podem ser caracterizadas tanto por memória longa como por não-linearidade, como, por exemplo, séries de volatilidade financeira, taxas de inflação e de câmbio. A Literatura afirma que modelos de memória longa, de quebras estruturais ou de mudança de regime podem capturar as mesmas características empíricas de uma mesma série de dados. Parece, portanto, interessante tentar incluir memória longa e não-linearidade em um único modelo, a fim de podermos verificar a importância relativa de cada fator. O objetivo deste trabalho é apresentar duas extensões do processo de memória longa, o FI-BREAK e o FI-STAR, que envolvem não-linearidades na série, ou seja, quebras estruturais e mudança de regime. 


\section{Abstract}

Many time series may have either long memory or non-linearity properties as, for example, financial volatility series, inflation and exchange rates. The Literacy says that long memory models, structural breaks or regime-switching models might have the same empirical properties from a single data series. Therefore, it seems interesting try to include many different empirical properties of a time series into one single model. This paper aims to show two extensions of the long memory process, the FI-BREAK and FI-STAR model, which have structural breaks and regime-switching properties. 


\section{Capítulo 1}

\section{Introdução}

Diversas séries temporais, inclusive séries financeiras, podem ser caracterizadas tanto por memória longa como por não-linearidade. Por exemplo, taxas de inflação, taxas de câmbio e algumas séries de volatilidade financeira (ver Bos et al. (1999)).

Alguns estudos, como o de Granger \& Hyung (2001) e Diebold \& Inoue (2001), mostraram que uma aparente memória longa pode ser negligenciada por mudanças estruturais em uma série temporal, enquanto outros estudos indicam exatamente o efeito reverso.

Granger \& Teräsvirta (1999) e Davidson (2000) demonstram que modelos nãolineares podem gerar séries temporais que podem ser ajustadas utilizando modelos de memória longa. Por outro lado, dados gerados a partir de modelos de memória longa podem parecer ter propriedades não-lineares (Andersson et. al (1999)).

Assim, os resultados empíricos para algumas séries temporais não devem ser vistos como surpresa caso modelos de memória longa e de quebra estrutural (ou de quebra de regime) capturarem as mesmas características empíricas. Parece, portanto, interessante tentar capturar ambas as características, para poder verificar a importância relativa de cada fator.

Vamos explorar a possibilidade de que o modelo de quebra estrutural (ou modelo BREAK) e o processo $\operatorname{ARFIMA}(p, d, q)$ de memória longa possam ser 
resumidos em um único modelo, o modelo FI-BREAK; vamos também tentar capturar a característica de mudança de regime (modelo STAR) e o processo $\operatorname{ARFIMA}(p, d, q)$ de memória longa em um único modelo, o modelo FI-STAR .

O objetivo deste trabalho é apresentar duas extensões do processo de memória longa, o FI-BREAK e o FI-STAR, que envolvem não-linearidades na série, ou seja, quebras estruturais e mudança de regime.

No Capítulo 2, iremos apresentar o modelo de memória longa, os processos $\operatorname{ARFIMA}(\mathrm{p}, \mathrm{d}, \mathrm{q})$, seus testes e principais métodos de estimação. No Capítulo 3, serão discutidos dois modelos de quebras estruturais, o STOPBREAK sugerido por Engle \& Smith (1999) e o método de minimização seqüencial da soma dos quadrados dos resíduos, proposto por Bai \& Perron (2003). No Capítulo 4, iremos abordar a questão da mudança de regime, os modelos associados SETAR e STAR, os testes de não-linearidade e de mudança de regime e o teste de não-linearidade restante.

No Capítulo 5, apresentaremos a extensão do modelo de memória longa com quebras estruturais, o FI-BREAK, e com mudança de regime, o FI-STAR. Para ambos os modelos, apresentamos os métodos de estimação e de identificação.

No Capítulo 6, apresentaremos a aplicação dos dois modelos a séries reais. Para o FI-STAR, modelamos a taxa de desemprego norte-americana dessasonalizada, de janeiro de 1969 a junho de 2004 e a série de retornos absolutos diários do IBOVESPA, de 03.01.1995 a 22.11.2000. Para o FI-BREAK, utilizamos a série de retornos mensais da Treasure Bill americana, de janeiro de 1934 a dezembro de 2003 e a série do índice de preços do trigo de Beveridge, de 1500 a 1845. Finalmente, no Capítulo 7 discutimos as conclusões obtidas com o trabalho. 


\section{Capítulo 2}

\section{Modelos de Memória Longa}

\subsection{Introdução}

Algumas séries temporais apresentam dependência significativa entre observações distanciadas por um longo período de tempo, tais como algumas séries econômicas e hidrológicas. Essas séries, por sua vez, possuem a propriedade de memória longa e muitas vezes não são ajustadas por modelos que pressupõem a significância das correlações entre observações distantes.

A partir da constatação desse problema, foram feitos vários estudos das características dessas séries para que fosse possível encontrar modelos capazes de representar adequadamente o processo de memória longa.

Verificou-se que uma das características principais dessas séries é o decaimento hiperbólico da função de autocorrelação. Em outras palavras, um processo de memória longa é aquele em que a função de autocorrelação $\rho_{j}$ decresce a uma taxa lenta (hiperbólica) para zero, ou seja,

$$
\rho_{j} \sim C_{j}^{2 d-1}, j \rightarrow \infty
$$

em que $C>0$ e $0<d<1$. 
Outra característica de séries com memória longa é que as autocorrelações da série original indicam "não-estacionariedade", ao passo que a série diferenciada vai ser "super-diferenciada".

O processo de memória longa foi introduzido por Hurst $(1951,1957)$. Posteriormente, esse tipo de séries temporais foi analisado por Mandelbrot \& Ness (1968), seguido por Granger \& Joyeux (1980) e Hosking (1981), que introduziram o modelo ARIMA fracionário (ou ARFIMA), que é uma generalização do modelo ARIMA. Geweke \& Porter-Hudak (1983) mostraram a equivalência entre o ARIMA e o ARFIMA.

Na década de 80, alguns estudos notaram que há evidências que processos de memória longa descrevem de modo satisfatório alguns dados financeiros, tais como taxas de juros e de inflação (ver Baillie (1996)). Estudos recentes da modelagem da volatilidade de ativos financeiros mostram que tais processos são muito úteis.

A Figura 2.1 abaixo mostra um exemplo de processo de memória longa, a série de índice anual de preços do trigo de Beveridge (de 1500 a 1869) e sua respectiva função de autocorrelação amostral.

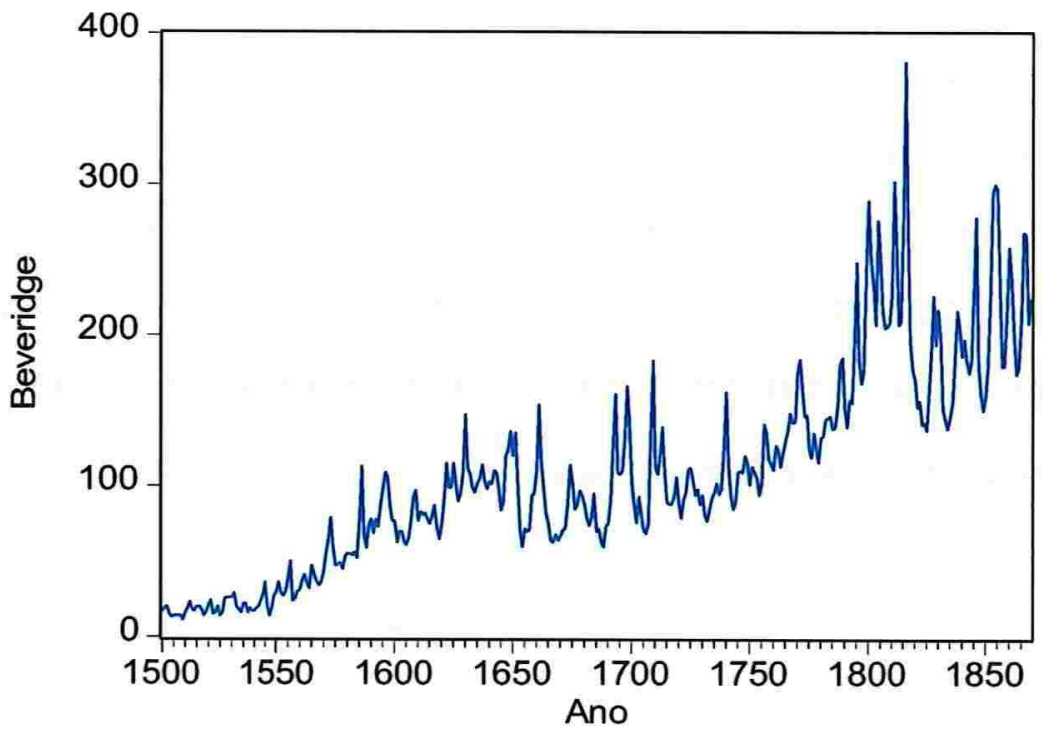




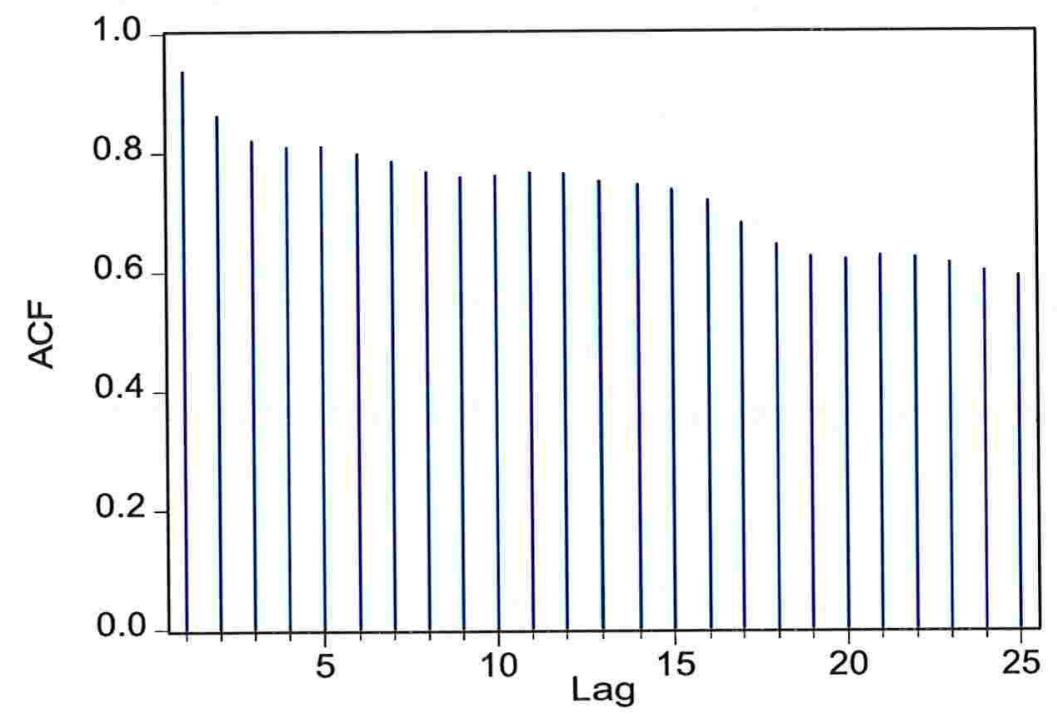

Figura 2.1 - Série de índices de preços de trigo de Beveridge (1500-1869) e fac amostral

\subsection{Modelos ARFIMA}

Seja $\left\{X_{t}\right\}$ uma série temporal. Um processo no tempo discreto ARIMA $(0,1,0)$ é modelado por $X_{t}=X_{t-1}+a_{t}$, em que $a_{t}$ é ruído branco, ou seja, variáveis aleatórias não-correlacionadas e identicamente distribuídas, para $t=1,2 \ldots$ Neste caso, à primeira diferença de $\left\{X_{t}\right\}$ é o processo ruído branco no tempo discreto $\left\{a_{t}\right\}$. Vamos assumir daqui em diante, por simplicidade, que $E\left(X_{t}\right)=0$.

Para qualquer número real $d>-1$, o operador de diferença fracionária é dado por :

$$
\nabla^{d}=(1-L)^{d}=\sum_{k=0}^{\infty}\left(\begin{array}{l}
d \\
k
\end{array}\right)(-L)^{k}=1-d L+\frac{1}{2 !} d(d-1) L^{2}-\frac{1}{3 !} d(d-1)(d-2) L^{3}+\ldots
$$

Definição 2.1: Chamamos de processo ruído branco de diferença fracionária, $X_{t}$, com parâmetro $d,-\frac{1}{2}<d<\frac{1}{2}$, a $d$-ésima diferença fracionária do processo ruído branco no tempo discreto, isto é, $X_{t}=\nabla^{-d} a_{t}$ ou $\nabla^{d} X_{t}=a_{t}$, em que $a_{t}$ é um 
processo ruído branco de média zero e variância $\sigma_{a}{ }^{2}$. O processo ruído branco de diferença fracionária $X_{t}$ é equivalente a um ARIMA $(0, \mathrm{~d}, 0) \operatorname{com} d \in\left(-\frac{1}{2}, \frac{1}{2}\right)$, que denominaremos de processo ARFIMA $(0, \mathrm{~d}, 0) .{ }^{1}$

Definição 2.2: $\left\{X_{t}\right\}$ é um processo auto-regressivo fracionário integrado de médias móveis, ou $\operatorname{ARFIMA}(\mathrm{p}, \mathrm{d}, \mathrm{q}) \operatorname{com}|d|<\frac{1}{2}$, se $\left\{X_{t}\right\}$ satisfizer a equação

$$
\phi(L)(1-L)^{d} X_{t}=\theta(L) a_{t}
$$

em que $\left\{a_{t}\right\}$ é um ruído branco e $\phi(L)$ e $\theta(L)$ são polinômios em $L$ de graus $p$ e $q$, respectivamente. Vamos verificar algumas propriedades do processo $\left\{X_{t}\right\}$.

Resultado 2.1: Seja $\left\{X_{t}\right\}$ um processo ARFIMA $(0, d, 0)$.

(a) Quando $d<\frac{1}{2},\left\{X_{t}\right\}$ é um processo estacionário e possui representação de médias-móveis infinita:

$$
X_{t}=\psi(L) a_{t}=\sum_{k=0}^{\infty} \varphi_{k} a_{t-k}
$$

em que

$$
\psi_{k}=\frac{d(1+d) \ldots(k-1+d)}{k !}=\frac{(k+d-1) !}{k !(d-1) !} .
$$

Como $\Gamma(d+k)=d(d+1) \ldots(d+k-1) / \Gamma(d), \quad \Gamma(k+1)=k !$ е $\Gamma(d)=(d-1)$ !, podemos escrever

\footnotetext{
${ }^{1}$ Do inglês "Auto-Regressive Fractionally Integrated Moving-Average";
} 


$$
\psi_{k}=\frac{\Gamma(k+d)}{\Gamma(k+1) \Gamma(d)},
$$

$\operatorname{com} \psi_{k} \sim \frac{k^{d-1}}{(d-1) !}$ quando $k \rightarrow \infty$.

(b) Quando $d>-\frac{1}{2},\left\{X_{t}\right\}$ é invertível e possui representação autoregressiva infinita

$$
\pi(L) X_{t}=a_{t}=\sum_{k=0}^{\infty} \pi_{k} X_{t-k}
$$

em que

$$
\pi_{k}=\frac{-d(1-d) \ldots(k-1-d)}{k !}=\frac{(k-d-1) !}{k !(-d-1) !} .
$$

Como $\quad \Gamma(k-d)=(k-d-1) \ldots(1-d)(-d) \Gamma(-d), \quad \Gamma(k+1)=k !$

$\Gamma(-d)=(-d-1)$ ! podemos escrever

$$
\pi_{k}=\frac{\Gamma(k-d)}{\Gamma(-d) \Gamma(k+1)}
$$

$\operatorname{com} \psi_{k} \sim \frac{k}{(-d-1) !}^{-d-1}$ quando $k \rightarrow \infty$.

Nos próximos itens assumiremos que $|d|<\frac{1}{2}$.

(c) A densidade espectral de $\left\{X_{t}\right\}$ é dada por

$$
f(\lambda)=\frac{\sigma_{a}^{2}}{2 \pi}\left(2 \operatorname{sen} \frac{1}{2} \lambda\right)^{-2 d}
$$

para $0<\lambda \leq \pi$ e $f(\lambda) \sim \lambda^{-2 d}$ quando $\lambda \rightarrow 0$.

(d) A função de autocovariância de $\left\{X_{l}\right\}$ é dada por 


$$
\gamma_{k}=E\left(X_{t} X_{t-k}\right)=\frac{(-1)^{k}(-2 d) !}{(k-d) !(-k-d) !} \sigma_{a}{ }^{2}
$$

e a função de autocorrelação de $\left\{X_{t}\right\}$

$$
\begin{aligned}
\rho_{k} & =\frac{\gamma_{k}}{\gamma_{0}}=\frac{(-d) !(k+d-1) !}{(d-1) !(k-d) !} \quad(k=0, \pm 1, \ldots) \\
& =\prod_{0<k \leq h} \frac{k-1+d}{k-d}, h=1,2, \ldots
\end{aligned}
$$

Em particular, $\gamma_{0}=\frac{(-2 d) !}{(-d) !^{2}}$ e $\rho_{1}=\frac{d}{(1-d)}$. Quando $k \rightarrow \infty$

$$
\rho_{k} \sim \frac{(-d) !}{(d-1) !} k^{2 d-1}
$$

Para mais detalhes, ver Hosking (1981) e Morettin e Toloi (2006). Portanto, pelo Resultado 2.1, se $-\frac{1}{2}<d<\frac{1}{2}$ o processo $\left\{X_{t}\right\}$ é estacionário e invertível. Além disso, $\varphi_{k}$ e $\pi_{k}$ decaem hiperbolicamente. O comportamento do espectro nas freqüências baixas indica que, para $d>0,\left\{X_{t}\right\}$ é um processo de memória longa, que também pode ser caracterizado pela queda hiperbólica da função de autocorrelação.

Observação: No processo $\operatorname{ARFIMA}(0, \mathrm{~d}, 0)$ o parâmetro $d$ pode assumir qualquer valor real. Nesse caso, o processo pode ser diferenciado um número finito de vezes até que o processo resultante tenha $|d|<1$, e será estacionário e invertível se $|d|<\frac{1}{2}$. Logo, $\nabla^{m}\left\{X_{1}\right\}$ será um processo ARFIMA(0,d,0) estacionário e invertível se $\nabla^{d} \nabla^{m} X_{t}=a_{t}$, em que $|d|<\frac{1}{2}$ e $m \in \mathrm{N}$. 
Vamos analisar algumas características do processo $\operatorname{ARFIMA}(0, \mathrm{~d}, 0)$ com relação a alguns valores que $d$ assume no intervalo $\left[-\frac{1}{2}, \frac{1}{2}\right]$ :

(a) Para $0<d<\frac{1}{2}$, o processo $\operatorname{ARFIMA}(0, \mathrm{~d}, 0)$ é estacionário de memória longa. As autocorrelações e autocorrelações parciais de $\left\{X_{t}\right\}$ são todas positivas e decaem monotonicamente para zero e $\sum_{k=-\infty}^{\infty}\left|\rho_{k}\right|=\infty$. A densidade espectral de $\left\{X_{t}\right\}$ é concentrada nas freqüências baixas: $f(\lambda)$ é uma função decrescente de $\lambda$ e $f(\lambda) \rightarrow \infty$ quando $\lambda \rightarrow 0$, mas é integrável.

(b) Para $d=0$, o processo ARFIMA $(0,0,0)$ é ruído branco com autocorrelações zero e densidade espectral constante.

(c) Para $-\frac{1}{2}<d<0$, o processo $\operatorname{ARFIMA}(0, \mathrm{~d}, 0)$ tem memória intermediária. As autocorrelações e autocorrelações parciais do processo são todas negativas, exceto $\rho_{0}=1$, e decaem monotonicamente e hiperbolicamente para zero e $\sum_{k=-\infty}^{\infty}\left|\rho_{k}\right|<\infty$. A densidade espectral é dominada por componentes de alta freqüência: $f(\lambda)$ é uma função crescente de $\lambda$ e $f(\lambda)=0$ quando $\lambda \rightarrow 0$, embora tenha gradiente $+\infty$.

Algumas vezes, utilizar o modelo $\operatorname{ARFIMA}(0, \mathrm{~d}, 0)$ em séries com comportamento de memória longa pode não ser satisfatório, pois alguma destas séries também podem apresentar um comportamento de memória curta. Então, o ideal seria encontrar um modelo capaz de explicar o comportamento de memória curta e que mantenha o decaimento hiperbólico da função de autocorrelação. Uma extensão do processo $\operatorname{ARFIMA}(0, \mathrm{~d}, 0)$ que possui essas propriedades é o processo que combina diferença fracionária com modelos Box \& Jenkins. 
Resultado 2.2: $\left\{Y_{t}\right\}$ é um processo $\operatorname{ARFIMA}(\mathrm{p}, \mathrm{d}, \mathrm{q})$ com $d \in\left(-\frac{1}{2}, \frac{1}{2}\right)$, se $\left\{Y_{t}\right\}$ for estacionário e satisfizer a equação

$$
\phi(L) \nabla^{d} Y_{\imath}=\theta(L) a_{t}
$$

em que $\left\{a_{t}\right\}$ é um ruído branco e $\phi$ e $\theta$ são polinômios em $L$ de graus $p$ e $q$, respectivamente.

Escolheremos essa família de processos para fins de modelagem das séries com comportamento de memória longa porque o efeito do parâmetro $d$ em observações distantes decai hiperbolicamente conforme a distância aumenta, explicando a estrutura de correlação de ordens altas da série, enquanto os efeitos dos parâmetros $\phi$ e $\theta$ decaem exponencialmente, explicando a estrutura de correlação de ordens baixas.

Caso o efeito dos parâmetros $\phi$ e $\theta$ não seja significativo para observações muito distantes, espera-se que o comportamento de memória longa de um $\operatorname{ARFIMA}(p, d, q)$ seja semelhante ao de um $\operatorname{ARFIMA}(0, d, 0)$, para o mesmo valor de $d$.

Resultado 2.3 (Hosking(1981)): Seja $\left\{Y_{l}\right\}$ um ARFIMA(p,d,q), então

(a) $\left\{Y_{t}\right\}$ é estacionário se $d<\frac{1}{2}$ e se todas as raízes da equação $\phi(z)=0$ estiverem fora do círculo unitário;

(b) $\left\{Y_{t}\right\}$ é invertível se $d>-\frac{1}{2}$ e se todas as raízes da equação $\theta(z)=0$ estiverem fora do círculo unitário.

Se $\left\{Y_{t}\right\}$ for estacionário e invertível, com função de densidade espectral $f(\lambda)$ e função de autocorrelação $\rho_{k}$, então

(a) $\lim _{\lambda \rightarrow 0} \lambda^{2 d} f(\lambda)$ existe e é finito; 
(b) $\lim _{k \rightarrow \infty} k^{1-2 d} \rho_{k}$ existe e é finito.

\subsection{Estimação de Modelos ARFIMA}

Os principais métodos de estimação do parâmetro de diferença fracionária, $d$, de modelos ARFIMA(p,d,q) dividem-se em três grupos:

(i) Métodos da função de autocorrelação, que foram elaborados para estimar o parâmetro $d$ somente em modelos ARFIMA(0,d,0). Porém, eles podem ser aplicados para estimar um $\operatorname{ARFIMA}(\mathrm{p}, \mathrm{d}, \mathrm{q})$ por meio de um algoritmo de estimação iterativo. Eles têm como base a relação entre $d$ e a função de autocorrelação ou de autocorrelação parcial de um processo ARFIMA $(0, d, 0)$. Dentre eles se destacam o Método da Função de Autocorrelação de Ordem 1 (Gupta \& Kumar (1991)), o Método da Função de Autocorrelação Generalizado (Gupta \& Ji (1992)) e o Método da Função de Autocorrelação Parcial (Vinod (1991));

(ii) Métodos de regressão usando o periodograma, que foram elaborados para estimar $d$ em modelos ARFIMA(p,d,q). Esses métodos se baseiam na relação entre a função de densidade espectral de um $\operatorname{ARFIMA}(p, d, q)$ e a de um $\operatorname{ARMA}(\mathrm{p}, \mathrm{q})$. Destacam-se o Método de Regressão usando o Periodograma (Geweke \& Porter-Hudak (1983)) e Método de Regressão usando o Periodograma Suavizado (Reisen (1994)). O problema desses métodos é que eles não estimam os parâmetros simultaneamente; $\mathrm{e}$

(iii) Métodos de Máxima Verossimilhança e Máxima Verossimilhança Aproximada, que são os únicos que estimam simultaneamente todos parâmetros do modelo ARFIMA(p,d,q). 
Apresentaremos a seguir os métodos de máxima verossimilhança e de máxima verossimilhança aproximada, que iremos usar no Capítulo 5.

\subsection{Método de Máxima Verossimilhança}

O estudo do estimador de máxima verossimilhança (EMV) para observações dependentes é vasto. Uma série temporal com comportamento de memória longa é classificada como uma seqüência de forte dependência e, portanto, o estimador de máxima verossimilhança para esse processo é um caso particular do citado anteriormente.

Para determinar o EMV para seqüências de forte dependência, Fox \& Taqqu (1986) aplicaram os resultados já obtidos para o caso de observações dependentes e apresentaram as condições que permitem que o EMV encontrado seja consistente e tenha distribuição assintótica normal. Essas condições são satisfeitas pelos modelos $\operatorname{ARFIMA}(\mathrm{p}, \mathrm{d}, \mathrm{q})$ e ruído Gaussiano fracionário.

A função de verossimilhança de $Z=\left(Z_{1}, \ldots, Z_{n}\right)$ proveniente de um processo $\operatorname{ARFIMA}(\mathrm{p}, \mathrm{d}, \mathrm{q})$ pode ser expressa na forma

$$
L\left(\eta, \sigma_{a}^{2}\right)=\left(2 \pi \sigma_{a}^{2}\right)^{-n / 2}\left(r_{0} \ldots r_{n-1}\right)^{\frac{-1}{2}} \exp \left[-\frac{1}{2 \sigma_{a}^{2}} \sum_{j=1}^{n}\left(Z_{j}-\hat{Z}_{j}\right)^{2} / r_{j-1}\right],
$$

em que $\eta=\left(d, \phi_{1}, \ldots, \phi_{p}, \theta_{1}, \ldots, \theta_{q}\right), \hat{Z}_{j}, j=1, \ldots, n$, são as previsões um passo à frente e $r_{j-1}=\left(\sigma_{a}^{2}\right)^{-1} E\left(Z_{j}-\hat{Z}_{j}\right)^{2}$.

Os estimadores de máxima verossimilhança são dados por

$$
\sigma_{M V}^{2}=n^{-1} S\left(\hat{\eta}_{M V}\right),
$$

em que

$$
S\left(\hat{\eta}_{M V}\right)=\sum_{j=1}^{n} \frac{\left(Z_{j}-\hat{Z}_{j}\right)^{2}}{r_{j-1}}
$$


e $\hat{\eta}_{M V}$ é o valor de $\eta$ que minimiza

$$
l(\eta)=\ln (S(\eta) / n)+n^{-1} \sum_{j=1}^{n} \ln r_{j-1} .
$$

Porém, o cálculo de $l(\eta)$ é muito lento. Uma maneira alternativa seria considerar uma aproximação para $l(\eta)$ dada por

$$
l(\eta) \cong l_{*}(\eta)=\ln \frac{1}{n} \sum_{j} \frac{I_{n}\left(w_{j}\right)}{2 \pi f\left(w_{j} ; \eta\right)}
$$

em que

$$
I_{n}\left(w_{j}\right)=\frac{1}{n}\left|\sum_{i=1}^{n} Z_{1} e^{-i n w_{j}}\right|^{2}
$$

é o periodograma e,

$$
f\left(w_{j} ; \eta\right)=\frac{\sigma_{a}^{2}}{2 \pi} \frac{\left|1-\theta_{1} e^{-i w_{j}}-\ldots-\theta_{q} e^{-q i w_{j}}\right|^{2}}{\left|1-\phi_{1} e^{-i w_{j}}-\ldots-\phi_{p} e^{-p i w_{j}}\right|^{2}} \cdot\left|1-e^{-i w_{j}}\right|^{-2 d}
$$

é a função densidade espectral do processo $\left\{Z_{r_{1}}\right\}_{;} \sum_{j}$ é a soma sobre todas as freqüências de Fourier, $w_{j}=2 \pi j / n \in(-\pi, \pi]$.

Hannan (1973) e Fox \& Taqqu (1986) mostram que:

(i) o estimador $\hat{\eta}_{M V}$ que minimiza (2.16) é consistente;

(ii) se $d>0$,

$$
\hat{\eta}_{M V} \stackrel{D}{\rightarrow}\left(\eta, n^{-1} A^{-1}(\eta)\right)
$$

em que $A(\eta)$ é uma matriz de ordem $(p+q+1) \times(p+q+1)$ com $(j, k)$ ésimo elemento dado por

$$
A_{j k}(\eta)=\frac{1}{4 \pi} \int_{-\pi}^{\pi} \frac{\partial \ln f(\lambda ; \eta)}{\partial\left(\eta_{j}\right)} \frac{\partial \ln f(\lambda ; \eta)}{\partial\left(\eta_{k}\right)} d \lambda ;
$$

(iii) a variância $\sigma_{a}^{2}$ é estimada por 


$$
\sigma_{M V}^{2}=\frac{1}{n} \sum_{j} \frac{I_{n}\left(w_{j}\right)}{2 \pi f\left(w_{j} ; \hat{\eta}_{M V}\right)} .
$$

\subsection{Método de Máxima Verossimilhança Aproximada}

Como vimos anteriormente, o método de máxima verossimilhança estima o parâmetro $d$ de um $\operatorname{ARFIMA}(\mathrm{p}, \mathrm{d}, \mathrm{q})$ apenas para o intervalo estacionário $-\frac{1}{2}<d<\frac{1}{2}$. Beran (1995) sugeriu um novo método, em que qualquer real $d>-\frac{1}{2}$ pode ser estimado. Tal procedimento chamado de Método de Máxima Verossimilhança Aproximada ("Approximate Maximum Likelihood Method") é apresentado a seguir.

Seja $\left\{X_{t}\right\}$ uma série temporal com $E\left(X_{t}\right)=0 \mathrm{e}$

$$
\xi=\left(\sigma_{\varepsilon}^{2}, d, \phi_{1}, \ldots, \phi_{p}, \theta_{1}, \ldots, \theta_{q}\right)=\left(\sigma_{\varepsilon}^{2}, \eta\right)
$$

o vetor de parâmetros desconhecidos. Sejam $d=m+\delta$ em que $-\frac{1}{2}<\delta<\frac{1}{2}$ e $m \geq 0$ inteiro. Dessa forma, $m$ será o número de vezes que $X$, deve ser diferenciada para se tornar estacionária obtendo

$$
\phi(L)(1-L)^{m} Y_{t}=\theta(L) \varepsilon_{t}
$$

em que

$$
Y_{t}=(1-L)^{\delta} X_{t}
$$

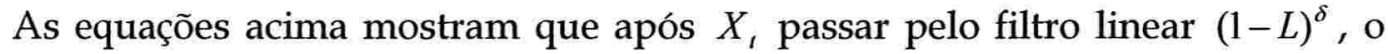
processo resultante é um $\operatorname{ARIMA}(\mathrm{p}, \mathrm{m}, \mathrm{q})$ com ordem de diferenciação $m$. Entretanto, $X_{t}$ pode também ser escrito na representação autoregressiva infinita

$$
\sum_{j=0}^{\infty} a_{j}(\eta) X_{t-j}=\varepsilon_{t}
$$

e os coeficientes $a_{j}(\eta)$ podem ser obtidos a partir da equação do operador de diferença fracionária: 


$$
(1-L)^{d}=\sum_{k=0}^{\infty} b_{k}(d) L^{k}
$$

com

$$
b_{k}(d)=(-1)^{k} \frac{\Gamma(d+1)}{\Gamma(k+1) \Gamma(d-k+1)} .
$$

Por exemplo, se tivermos um $\operatorname{ARIMA}(\mathrm{p}, \mathrm{m}, \mathrm{q}) \operatorname{com} p=q=0$, então $a_{j}(\eta)=a_{j}(d)$ são obtidos da equação (2.23) por

$$
a_{j}(d)=\sum_{k=0}^{\min (m, j)}(-1)^{k}\left(\begin{array}{l}
m \\
k
\end{array}\right) b_{j-k}(\delta) .
$$

Dadas as observações $X_{1}, \ldots, X_{n}$, as inovações $\varepsilon$, podem ser estimadas pelos resíduos

$$
e_{t}(\eta)=\sum_{j=0}^{t-1} a_{j}(\eta) X_{t-j}
$$

Os resíduos padrões são definidos por $r_{t}(\theta)=e_{t}(\eta) / \sqrt{\sigma_{\varepsilon}^{2}}$. Os erros seriam dados por

$$
\varepsilon_{t}(\eta)=\sum_{j=0}^{\infty} a_{j}(\eta) X_{t-j}
$$

e os erros padronizados por $v_{l}(\theta)=\varepsilon_{l}(\eta) / \sqrt{\sigma_{\varepsilon}^{2}}$. Assumindo que os parâmetros do ARMA são tais que as raízes de $\phi(x)$ e $\theta(x)$ estão fora do círculo unitário, Beran (1995) mostra que os $a_{j}(\eta)$ são continuamente diferenciáveis com relação a $\eta$ para $d>-\frac{1}{2}$. Dessa forma, podemos definir as derivadas

$$
\begin{gathered}
\dot{e}_{t}(\eta)=\left(\frac{\partial}{\partial \eta_{1}} e_{t}(\eta), \ldots, \frac{\partial}{\partial \eta_{p+q+1}} e_{l}(\eta)\right)^{T}, \\
\dot{r}_{t}(\eta)=\left(\frac{\partial}{\partial \xi_{1}} r_{i}(\xi), \ldots, \frac{\partial}{\partial \xi_{p+q+2}} r_{i}(\xi)\right)^{T}, \\
\dot{\varepsilon}_{t}(\eta)=\left(\frac{\partial}{\partial \eta_{1}} \varepsilon_{t}(\eta), \ldots, \frac{\partial}{\partial \eta_{p+q+1}} \varepsilon_{l}(\eta)\right)^{T},
\end{gathered}
$$




$$
\dot{v}_{t}(\eta)=\left(\frac{\partial}{\partial \xi_{1}} v_{t}(\xi), \ldots, \frac{\partial}{\partial \xi_{p+q+2}} v_{t}(\xi)\right)^{T} .
$$

Caso $E\left(X_{t}\right)$ seja desconhecida, os resíduos definidos acima precisam ser ajustados. Há várias maneiras para se fazer isso. Para $m=0$, basta subtrair a média amostral das observações. Porém, para dados não-estacionários, em que $m>0$, isso não é possível. Beran (1995) sugere a seguinte metodologia: primeiro, definimos

$$
U_{t}(m)=(1-L)^{m} X_{t} .
$$

de forma que a m-ésima diferença de $X_{t}$ é estacionária com valor esperado $E\left(X_{t}\right)=\mu$. Assim, $U_{t}$ é um $\operatorname{ARFIMA}(\mathrm{p}, \delta, \mathrm{q})$ estacionáro com média $\mu$ e é ergódico de forma que a média amostral

$$
\bar{U}=\frac{1}{n-m} \sum_{t=m+1}^{n} U_{t}
$$

é um estimador consistente de $\mu$. Dessa forma, podemos definir os resíduos ajustados como

$$
e_{t}(\eta)=\sum_{j=0}^{t-1} a_{j}\left(\eta^{*}\right)\left(U_{t-j}-\bar{U}\right)
$$

em que $\eta=\left(d-m, \phi_{1}, \ldots, \phi_{p}, \theta_{1}, \ldots, \theta_{q}\right)=\left(\delta, \phi_{1}, \ldots, \phi_{p}, \theta_{1}, \ldots, \theta_{q}\right)$. A contrapartida populacional é dada por

$$
\varepsilon_{t}(\eta)=\sum_{j=0}^{\infty} a_{j}\left(\eta^{*}\right)\left(U_{t-j}-\mu\right) .
$$

Todas as outras partes definidas para $\mu=0$ podem ser definidas analogamente, ao substituir os resíduos não-ajustados pelos resíduos ajustados.

As representações autoregressivas (2.25) e (2.29) sugerem estimar $\xi$ pelo método de máxima verossimilhança aproximada: minimizar a soma dos quadrados dos resíduos

$$
S(\eta)=\sum_{i=2}^{n} e_{t}^{2}(\eta)
$$


com relação a ๆ e obter

$$
\hat{\xi}_{1}=\hat{\sigma}_{\varepsilon}^{2}=\frac{1}{n-1} S(\hat{\eta})
$$

em que $e_{t}(\eta)$ é definida pelas equações (2.30) ou (2.34). Isso resulta na resolução de $p+q+2$ equações não-lineares

$$
\sum_{t=2}^{n}\left\{r_{t}(\xi) \dot{r}_{t}(\xi)-c(\xi)\right\}=0
$$

em que $c(\xi)=E\left[v_{t}(\xi) \dot{v}_{t}(\xi)\right]$. Note que $c_{1}(\xi)=-2\left(\xi_{1}\right)^{-1} E\left[v_{l}^{2}\right]=-1 / 2 \xi_{1} \quad$ e $c_{j}(\xi)=0$ para $j>1$.

Resultado 2.4 (Beran (1995)): Sejam $\hat{\xi}$ as soluções das equações (2.33) e defina $\xi^{*}=\left(\sigma_{\varepsilon}^{2}, \eta^{*}\right)^{T}=\left(\sigma_{\varepsilon}^{2}, \delta, \eta_{2}, \ldots, \eta_{p+q+1}\right)^{T}$. Isso significa que $\xi_{2}=d=m+\delta$ é substituída por $\xi_{2}^{*}=\delta$. Então, à medida que $n \rightarrow \infty$

(a) $\hat{\xi}$ converge quase-certamente para o verdadeiro valor $\xi$ e

(b) $n^{1 / 2}(\hat{\xi}-\xi)$ converge em distribuição para um vetor aletório normal com média 0 e matriz de covariâncias

$$
V=2 D^{-1}
$$

em que

$$
D_{i j}=(2 \pi)^{-1}\left[\int _ { - \pi } ^ { \pi } \frac { \partial } { \partial \xi _ { i } } \left\{\left.\log (f(x)\} \frac{\partial}{\partial \xi_{j}}\{\log (f(x)\} d x]\right|_{\xi=\xi^{*}} .\right.\right.
$$

\subsection{Testes de Memória Longa}

Além da análise da função da autocorrelação de uma série temporal, podemos realizar testes de hipóteses para verificar se essa série possui memória longa. Vamos apresentar nesta seção dois testes de memória longa: o teste da estatística R/S e o teste GPH. 


\subsubsection{Teste da Estatística R/S}

Proposta por Hurst (1951), com o nome "rescaled range" (ou "range over standard deviation"), a estatística R/S é a distância de somas parciais dos desvios de uma série temporal em relação à sua média, dividida pelo seu desvio-padrão.

Dada uma série temporal $y_{t}$, para $t=1, \ldots, T$, a estatística R/S é definida como:

$$
Q_{T}=\frac{1}{S_{T}}\left[\max _{1 \leq k \leq T} \sum_{j=1}^{k}\left(y_{j}-\bar{y}\right)-\min _{1 \leq k \leq T} \sum_{j=1}^{k}\left(y_{j}-\bar{y}\right)\right],
$$

em que $\bar{y}$ é a média amostral e $S_{T}$ é o desvio-padrão amostral. Pode-se demonstrar que se $y_{t}$ é gaussiano, então $Q_{T} / \sqrt{T}$ converge fracamente para uma v.a. que está no domínio de atração de uma ponte browniana ${ }^{2}$ no intervalo unitário. Lo (1991) fornece os quantis dessa variável limite; ele nota que a estatística definida por (2.35) não é robusta à dependência de memória curta e propõe substituir $Q_{T}$ por

$$
\widetilde{Q}_{T}=\frac{1}{\hat{\sigma}_{T}(q)}\left[\max _{1 \leq k \leq T} \sum_{j=1}^{k}\left(y_{j}-\bar{y}\right)-\min _{1 \leq k \leq T} \sum_{j=1}^{k}\left(y_{j}-\bar{y}\right)\right],
$$

em que $\hat{\sigma}_{T}(q)$ é a raiz quadrada do estimador da variância de longo prazo de Newey-West com largura de faixa $q$, dado por

$$
\hat{\sigma}_{T}^{2}(q)=S_{T}^{2}\left(1+\frac{2}{n} \sum_{j=1}^{q} w_{q j} r_{j}\right)
$$

sendo $w_{q j}=1-j /(q+1), q<n$ e $r_{j}$ são as autocorrelações amostrais usuais de $y_{i}$.

Newey \& West (1987) sugerem escolher $q=\left[4(n / 100)^{2 / 9}\right]$.

Se o processo $y_{1}$ não tiver memória longa, a estatística R/S converge para sua distribuição limite à taxa $n^{1 / 2}$, mas se há memória longa na série, a taxa de convergência será igual a $n^{H}, \operatorname{com} H=d+1 / 2$.

2 Seja $\{X(t), t \geq 0\}$ um Movimento Browniano, então o processo estocástico condicional $\{X(t), 0 \leq t \leq 1 \mid X(1)=0\}$ é uma Ponte Browniana. Pode-se mostrar que o processo estocástico $\{Z(t), 0 \leq t \leq 1\}$ é uma Ponte Browniana quando $Z(t)=X(t)-t X(1)$. 
Isso sugere a construção de gráficos (na escala log-log) de R/S contra o tamanho amostral. Para uma série com memória curta, os pontos deve estar ao longo de uma reta com inclinação $1 / 2$, enquanto que para uma série com memória longa, a reta deve ter inclinação $H>1 / 2$, para grandes amostras.

Para a construção desse gráfico, consideram-se os valores de R/S contra $k_{i}$, para $k_{i}=f k_{i-1}, i=2, \ldots, s, k_{1}$ inicialmente grande e $f$ um fator conveniente. Por exemplo, divide-se a amostra em $\left[n / k_{i}\right]$ blocos.

\subsubsection{Teste GPH}

Geweke \& Porter-Hudak (1983) apresentaram um método semi-paramétrico para testar a presença de memória longa, baseando-se na seguinte representação de uma série temporal com memória longa

$$
(1-L)^{d}\left(y_{t}-\mu\right)=u_{\imath}
$$

A densidade espectral de um processo $y_{t}$ fracionariamente integrado é dada por

$$
f(\omega)=\left[4 \operatorname{sen}^{2}\left(\frac{\omega}{2}\right)\right]^{-d} f_{u}(\omega),
$$

em que $f_{u}(\omega)$ é a densidade espectral correspondente a $u_{t}$. O parâmetro de diferença fracionária $d$ pode ser estimado pela seguinte regressão:

$$
\ln f\left(\omega_{j}\right)=\beta-d \ln \left[4 \operatorname{sen}^{2}\left(\frac{\omega}{2}\right)\right]+e_{j},
$$

para $\omega_{j}=\frac{j}{T}, j=1,2, \ldots, n_{f}(T)$. Geweke \& Porter-Hudak (1993) demonstram que usando como uma estimativa de $f\left(\omega_{j}\right)$ o periodograma, o estimador de mínimos quadrados $\hat{d}$, obtido em (2.39) tem distribuição normal assintótica se $n_{f}(T)=T^{\alpha}$, com $0<\alpha<1$. Assim, 


$$
\hat{d}_{\rightarrow}^{D} N\left(d, \frac{\pi^{2}}{6 \sum_{j=1}^{n_{f}}\left(U_{j}-\bar{U}\right)^{2}}\right),
$$

em que

$$
U_{j}=\ln \left[4 \operatorname{sen}^{2}\left(\frac{\omega_{j}}{2}\right)\right]
$$

e $\bar{U}$ é a média amostral de $U_{j}, j=1, \ldots, n_{f}$. Sob a hipótese nula de que não há memória longa $(d=0)$, a estatística t dada por

$$
t_{d=0}=\hat{d} \cdot\left(\frac{\pi^{2}}{6 \sum_{j=1}^{n_{f}}\left(U_{j}-\bar{U}\right)^{2}}\right)^{-1 / 2},
$$

tem distribuição assintótica $N(0,1)$. Dessa forma, utilizando (2.40) podemos testar a hipótese nula de que não há memória longa contra a alternativa de que $d \neq 0$. 


\section{Capítulo 3}

\section{Modelos de Quebra Estrutural}

\subsection{Introdução}

Definimos como choque em uma série temporal, $\left\{y_{1}\right\}$, um valor observado de $\left\{y_{1}\right\}$ muito distante de sua média não-condicional. A análise de séries temporais traça uma linha tênue que separa os choques que possuem efeito permanente daqueles que não têm, ou seja, os choques que podem alterar ou não a média nãocondicional da série $\left\{y_{1}\right\}$. Por exemplo, enquanto em um processo $\operatorname{AR}(1)$ estacionário todos os choques são transitórios, em um passeio aleatório todos os choques são permanentes. À medida que a raiz do polinômio auto-regressivo de um $\mathrm{AR}(1)$ se aproxima de um, a taxa pela qual os choques caem decresce, mas os choques continuam sendo transitórios.

O conceito de variação dos impactos permanentes dos choques está ligado ao conceito de mudança estrutural. Toda vez que um choque ou parte dele possuir um efeito permanente, pode-se interpretá-lo como um tipo específico de quebra estrutural. Logo, uma quebra ou mudança estrutural é uma mudança na média não-condicional de uma série causada por um choque ou parte dele. Dessa forma, sob essa definição, um passeio aleatório possui uma quebra em todos os períodos, mas um processo ARMA estacionário não possui nenhuma quebra estrutural. 
Processos como autoregressão limiar, "threshold autoregression" (Tong (1983)), também não possuem quebras estruturais. Os parâmetros mudam de valores, o que faz com que os choques decresçam a uma taxa diferente, mas mesmo assim eles continuam sendo transitórios.

Várias séries temporais como, por exemplo, taxas de câmbio, taxas de inflação e séries de volatilidade financeira, possuem quebras estruturais (ver Bos et al. (1999)). Além disso, ao realizarmos testes de raiz unitária, devemos tomar cuidado se a série possuir quebras estruturais, pois nesse caso as estatísticas dos testes de Dickey-Fuller e Phillips-Perron serão viesadas para a não-rejeição de uma raiz unitária (ver Enders (1995)).

Apresentaremos neste capítulo duas abordagens para tratar quebras estruturais. A primeira consiste no método de estimação seqüencial dos pontos de quebra estrutural de Bai \& Perron (2003), em que é mostrado um caso geral de mudança estrutural, tratando os pontos de quebras como variáveis desconhecidas a serem estimadas.

A segunda consiste no modelo STOPBREAK, proposto por Engle \& Smith (1999), que consiste em um processo de mudanças estruturais aleatórias que permite que essas mudanças sejam suaves, o que se ajusta melhor à análise empírica.

\subsection{Método de Estimação Seqüencial dos Pontos de Quebra Estrutural}

\subsubsection{Estimação Seqüiencial}

Introduzido por Bai \& Perron (1998), o método de estimação seqüencial dos pontos de quebra estrutural, considera múltiplas quebras estruturais em um modelo de regressão linear estimado ao minimizar a soma dos quadrados dos resíduos. As datas das quebras são consideradas variáveis desconhecidas. Esse 
método permite diversas formas de correlação serial e heterocedasticidade nos resíduos, variáveis dependentes defasadas, diferentes distribuições para os erros, além de considerar o caso mais geral de mudança estrutural parcial onde nem todos os parâmetros estão sujeitos a quebras.

Bai \& Perron (1998) apresentam o seguinte modelo de regressão linear múltipla com $m$ quebras (ou $m+1$ regimes):

$$
y_{t}=x_{t}^{\prime} \beta+z_{t}^{\prime} \delta_{j}+u_{\imath}, t=T_{j-1}+1, \ldots, T_{j}, j=1, \ldots ., m+1,
$$

usando a convenção de que $T_{0}=0$ e $T_{m+1}=T$. Nesse modelo, $y_{\imath}$ é a variável observável, $x_{t}(p \times 1)$ e $z_{t}(q \times 1)$ são os vetores de covariáveis, e $\beta$ e $\delta_{j}(j=1, \ldots, m$ +1) são os vetores de coeficientes correspondentes; $u$, é o termo de perturbação. Os pontos de quebra, $\left(T_{1}, \ldots, T_{m}\right)$, são tidos como desconhecidos. O objetivo desse modelo é estimar os coeficientes desconhecidos da regressão junto com os pontos de quebra, quando temos $T$ observações de $\left(y_{t}, x_{t}, z_{t}\right)$. Note que esse é um modelo de mudança estrutural parcial, pois $\beta$ não está sujeito a mudanças e é estimado usando a amostra inteira. No caso em que $p=0$, obtemos um modelo de mudança estrutural pura onde todos os coeficientes estão sujeitos a mudanças. A variância de $u_{t}$ não precisa ser constante. De fato, quebras na variância são permitidas desde que elas ocorram nas mesmas datas que as quebras nos parâmetros da regressão (para mais detalhes, ver Bai \& Perron (2003)).

A regressão linear múltipla (3.1) pode ser expressa na forma matricial como $Y=X \beta+\bar{Z} \delta+U, \quad$ em $\quad$ que $\quad Y=\left(y_{1}, \ldots, y_{T}\right)^{\prime}, \quad X=\left(x_{1}, \ldots, x_{T}\right)^{\prime}, \quad U=\left(u_{1}, \ldots, u_{T}\right)^{\prime}$, $\delta=\left(\delta_{1}^{\prime}, \delta_{2}^{\prime}, \ldots, \delta_{m+1}^{\prime}\right)^{\prime}$, e $\bar{Z}$ é a matriz com partições diagonais $Z$ na partição $m$ $\left(T_{1}, \ldots, T_{m}\right)$, ou seja, $\bar{Z}=\operatorname{diag}\left(Z_{1}, \ldots, Z_{m+1}\right)$ com $Z_{i}=\left(z_{T_{i-1}+1}, \ldots, z_{T_{i}}\right)^{\prime}$. Denotaremos o verdadeiro valor de um parâmetro com um 0 de sobrescrito. Particularmente, $\delta^{0}=\left(\delta_{1}^{0^{\circ}}, \ldots, \delta_{m+1}^{0^{0}}\right)^{\prime}$ e $\left(T_{1}^{0}, \ldots, T_{m}^{0}\right)$ são os valores verdadeiros dos parâmetros $\delta$ e dos pontos de quebra. A matriz $\bar{Z}^{0}$ é aquela cujas partições diagonais são $\mathrm{Z}$ em $\left(T_{1}^{0}, \ldots T_{m}^{0}\right)$. Assim, o processo gerador dos dados é assumido como 


$$
Y=X \beta^{0}+\bar{Z}^{0} \delta^{0}+U .
$$

O método de estimação considerado é baseado no princípio de mínimos quadrados. Para cada partição $m\left(T_{1}, \ldots, T_{m}\right)$, denotado por $\left\{T_{j}\right\}$, as estimativas de mínimos quadrados de $\beta$ e $\delta_{j}$ são obtidas ao minimizarmos a soma dos quadrados dos resíduos

$$
(Y-X \beta-\bar{Z} \delta)^{\prime}(Y-X \beta-\bar{Z} \delta)=\sum_{I=1}^{m+1} \sum_{i=T_{i-1}+1}^{T_{i}}\left[y_{t}-x_{t}^{\prime} \beta-z_{t}^{\prime} \delta_{i}\right]^{2} .
$$

Sejam $\hat{\beta}\left(\left\{T_{j}\right\}\right)$ e $\hat{\delta}\left(\left\{T_{j}\right\}\right)$ as estimativas resultantes baseadas na partição $m$ dada $\left(T_{1}, \ldots, T_{m}\right)$ denotada por $\left\{T_{j}\right\}$. Substituindo-as na função objetiva e denotando a soma dos quadrados dos resíduos resultante como $S_{T}\left(T_{1}, \ldots, T_{m}\right)$, os pontos de quebra resultantes $\left(\hat{T}_{1}, \ldots, \hat{T}_{m}\right)$ são tais que

$$
\left(\hat{T}_{1}, \ldots, \hat{T}_{m}\right)=\arg \min _{T_{1}, \ldots, T_{m}} S_{T}\left(T_{1}, \ldots, T_{m}\right),
$$

onde a minimização é feita em todas as partições $\left(T_{1}, \ldots, T_{m}\right)$ tais que $T_{i}-T_{i-1} \geq h$. Assim, os estimadores dos pontos de quebra são minimizadores globais da função objetiva. As estimativas dos parâmetros da regressão são as estimativas de mínimos quadrados na partição $m$ estimada $\left\{\hat{T}_{j}\right\}$, ou seja, $\hat{\beta}=\hat{\beta}\left(\left\{T_{j}\right\}\right)$ e $\hat{\delta}=\hat{\delta}\left(\left\{T_{j}\right\}\right)$. Como os pontos de quebra são parâmetros discretos e podem tomar apenas um número finito de valores, eles podem ser estimados por um grid search. Porém, esse método fica computacionalmente complicado quando $m>2$. Para contornar esse problema, Bai \& Perron (2003) apresentam um eficiente método discutido a seguir.

\subsubsection{Minimizadores Globais}

Para resolver o problema computacional mostrado na seção anterior, Bai \& Perron (2003) apresentam um algoritmo baseado no princípio de programação dinâmica que permite a computação de estimativas dos pontos de quebra como minimizadores globais da soma dos quadrados dos resíduos. Esse algoritmo usa 
no máximo $O\left(T^{2}\right)$ operações de mínimos quadrados para qualquer número $m$ de mudanças estruturais, ao invés de um grid search, que iria requerer operações de mínimos quadrados de $O\left(T^{m}\right)$. A base desse método foi apresentada por Guthery (1974), Bellman \& Roh (1969) e Fisher (1958).

Seja $T$ o tamanho da amostra, o número total de possíveis segmentos é no máximo igual a $T(T+1) / 2$ e, portanto, de ordem $O\left(T^{2}\right)$. Isso é mostrado na Figura 3.1 para o caso especial $\operatorname{com} T=25$ e $m=2$, onde o eixo vertical representa a data inicial de um segmento e o eixo horizontal denota a data final. Por exemplo, a entrada $(4,10)$ indica um segmento que começa na data 4 e termina na data 10 , tendo assim 7 observações. Cada entrada representa uma soma dos quadrados dos resíduos correspondente ao segmento associado. Também fixamos o tamanho do segmento igual a 5 no exemplo da Figura 3.1.

Na Figura 3.1, $x^{a}$ indica um segmento desconsiderado, pois ele deve ter pelo menos um comprimento igual a $5, x^{b}$ denota um segmento desconsiderado, pois não há espaço para 3 segmentos de magnitude 5 e $x^{c}$ representa um segmento desconsiderado porque de outra forma não haveria espaço para um segmento de ordem 5 antes deste. Por fim, o ponto • indica um segmento admissível. 


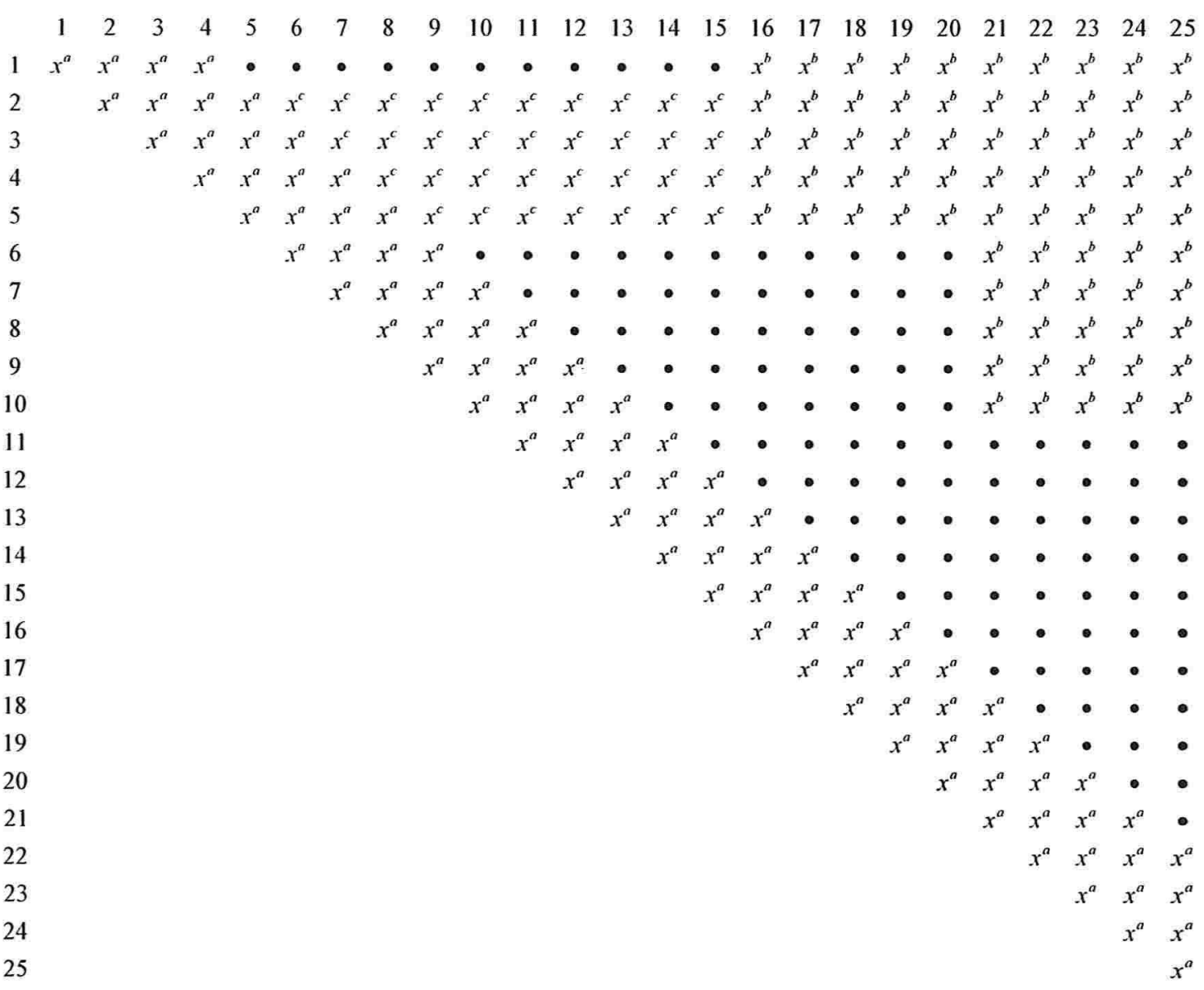

Figura 3.1 - Matriz Triangular da Soma dos Quadrados dos Resíduos com $T$ $=25, h=5$ e $m=2$

A soma dos quadrados dos resíduos global para qualquer partição $m\left(T_{1}, \ldots, T_{m}\right)$ e para qualquer valor de $m$ deve necessariamente ser uma combinação linear particular dessas $T(T+1) / 2$ soma dos quadrados dos resíduos. As estimativas das datas das quebras, na partição $m\left(\hat{T}_{1}, \ldots, \hat{T}_{m}\right)$, correspondem a esta combinação linear com o valor mínimo. O algoritmo de programação dinâmica pode ser visto como uma maneira eficiente para comparar possíveis combinações (correspondentes a 
diferentes partições $m$ ) para alcançar uma soma mínima global dos quadrados dos resíduos ${ }^{3}$.

Consideremos, em primeiro lugar, o caso de um modelo de mudança estrutural pura com a regressão dada por

$$
Y=\bar{Z} \delta+U
$$

Nesse caso, a computação das estimativas $\hat{\delta}, \hat{u}_{t}$ e $S_{T}\left(T_{1}, \ldots, T_{m}\right)$ pode ser feita aplicando MQO em cada segmento, sem restrições entre eles.

O número total de possíveis segmentos pode ser representado pela matriz triangular da soma dos quadrados dos resíduos, onde o eixo vertical representa a data inicial do segmento, enquanto o eixo horizontal denota a data final do segmento.

A computação da matriz triangular da soma dos quadrados dos resíduos para (3.4) pode ser feita usando uma fórmula de atualização que calcule os resíduos recursivos. Seja $v(i, j)$ o resíduo recursivo no tempo $j$ obtido usando uma amostra que começa na data $i$, e seja $S Q R(i, j)$ a soma dos quadrados dos resíduos obtidas aplicando mínimos quadrados em um segmento que começa na data $i$ e termina na data j. De acordo com Brown, Durbin \& Evans (1975), temos: $S Q R(i, j)=S Q R(i, j-1)+v(i, j)^{2}$. Toda informação relevante está contida nos valores $S Q R(i, j)$ para as combinações relevantes $(i, j)$. Note que o número de inversões de matrizes necessárias é de ordem $O(T)$.

Uma vez computadas a soma dos quadrados dos resíduos dos segmentos relevantes, a abordagem da programação dinâmicà pode ser usada, para avaliar qual partição alcança uma minimização global da soma geral dos quadrados dos resíduos. Esse método procede essencialmente através de um exame seqüencial de

\footnotetext{
${ }^{3}$ Segundo Bai \& Perron (2003), na prática, menos do que $T(T+1) / 2$ segmentos são permitidos. Pois, em primeiro lugar, alguma distância mínima, $h$, entre cada quebra deve ser imposta e o maior segmento deve ser curto o suficiente para permitir $m$ outros segmentos antes ou depois. Além disso, um segmento não pode começar em datas indo da $2^{\mathrm{a}}$ observação a $h$, pois assim nenhum segmento de comprimento mínimo $h$ poderia ser inserido no começo dos segmentos a serem considerados. Isso implica uma redução no número de segmentos a ser considerado.
} 
partições ótimas de uma quebra (ou de dois segmentos). Seja $S Q R\left(\left\{T_{r, n}\right\}\right)$ a soma dos quadrados dos resíduos associada à partição ótima contendo $r$ quebras usando as primeiras $n$ observações; a partição ótima resolve o seguinte problema recursivo:

$$
\operatorname{SQR}\left(\left\{T_{m, T}\right\}\right)=\min _{m h \leq j \leq T-h}\left\lfloor\operatorname{SQR}\left(\left\{T_{m-1, j}\right\}\right)+\operatorname{SQR}(j+1, T)\right\rfloor .
$$

O procedimento começa ao avaliar a partição de uma quebra ótima para todas as subamostras que permitem uma possível quebra que vai da observação $h$ (comprimento mínimo de um segmento) até a observação $T$ - $m h$. Dessa forma, o primeiro passo é obter um conjunto de $T-(m+1) h+1$ partições de quebras ótimas com suas somas dos quadrados dos resíduos associadas. Cada uma das partições ótimas corresponde a sub-amostras terminando em datas variando de $2 h$ a $T-(m-$ 1)h.

Vamos considerar agora o próximo passo que consiste em uma busca de partições ótimas com duas quebras. Tais partições têm datas finais variando de $3 h$ a $T-(m-2) h$. Para cada uma dessas possíveis datas finais, o procedimento olha em qual partição (obtida anteriormente) pode ser inserida para obter uma soma dos quadrados dos resíduos mínima. O resultado é um conjunto de $T-(m+1) h+1$ duas partições (ou três segmentos) de quebras ótimas. O método continua seqüencialmente até um conjunto de $T-(m+1) h+1$ ótimas $(m-1)$ partições de quebras forem obtidas com datas finas variando de $(m-1) h$ a $T-2 h$. O passo final é verificar qual dessas $(m-1)$ partições de quebras resulta em uma menor soma dos quadrados dos resíduos geral quando combinada com um segmento adicional.

Esse método pode, portanto, ser visto como uma atualização seqüencial de $T$ $(m+1) h+1$ segmentos em uma, duas ou até $m-1$ partições de quebras (ou em dois, três ou até $m$ segmentos); o último passo é criar uma única partição ótima com $m$ quebras (ou $m+1$ segmentos).

Segundo Bai \& Perron (2003), esse método é muito rápido, na prática, utilizando amostras dos tamanhos usuais. $\mathrm{Na}$ verdade, o maior custo 
computacional é a construção da matriz triangular da soma dos quadrados dos resíduos para todos os possíveis segmentos. A busca da partição m-ótima representa apenas uma adição marginal ao tempo computacional total. Isso significa que obter minimizadores globais com cinco ou dez quebras é apenas ligeiramente mais demorado do que com duas.

Esse método de programação dinâmica, para obter minimizadores globais da soma dos quadrados dos resíduos não pode ser diretamente aplicado para o caso do modelo de mudança estrutural parcial $(p>0)$. Isso porque a estimativa de $\beta$ associada com uma minimização global depende da partição ótima que estamos tentando obter. Ao contrário do modelo de mudança estrutural pura, pelo qual podemos escrever a regressão na forma (3.4), cada elemento da matriz triangular da soma dos quadrados dos resíduos depende da m-partição ótima final que estamos buscando.

Entretanto, segundo Bai \& Perron (2003), é possível usar um procedimento iterativo. Seja $\theta=\left(\delta, T_{1}, \ldots T_{m}\right)$, podemos escrever a soma dos quadrados dos resíduos como uma função dos vetores $\beta$ e $\theta$, isto é, $S Q R(\beta, \theta)$. Como foi discutido em Sargan (1964), podemos minimizar $\operatorname{SQR}(\beta, \theta)$ de uma forma iterativa. Em primeiro lugar, minimizamos $\operatorname{SQR}(\beta, \theta) \operatorname{com}$ relação a $\theta$, tomando $\beta$ fixo e depois minimizamos com relação a $\beta$ mantendo $\theta$ fixo, e iteramos. Cada iteração assegura uma redução na função objetiva (para mais detalhes, ver Sargan (1964)).

Note que o primeiro passo, de minimizar $\operatorname{SQR}(\beta, \theta)$ com relação a $\theta$ tomando $\beta$ fixo, corresponde a aplicar o algoritmo de programação dinâmica discutido acima com $y_{1}-x_{1}^{\prime} \beta$ como a variável dependente. Como $\beta$ é fixo, isso é um passo envolvendo um modelo de mudança estrutural pura. Seja $\theta^{*}=\left(\delta^{*},\left\{T^{*}\right\}\right)$ a estimativa associada a esse primeiro estágio $\left(\operatorname{com}\left\{T^{*}\right\}=\left(T_{1}^{*}, \ldots T_{m}^{*}\right)\right)$. A aplicação do método de Sargan (1964) sugere que o segundo passo seja uma regressão linear 
simples com $y_{t}-z_{l}^{\prime} \delta_{j}^{*}$ sendo a variável dependente para $t$ no regime $j(j=1, \ldots, m+$ 1), os regimes sendo definidos pela partição $\left\{T^{*}\right\}$.

Segundo Bai \& Perron (2003), ganhos de eficiência podem ser obtidos ao modificarmos o segundo passo. A idéia é apenas manter $\left\{T^{*}\right\}$ fixado e maximizar novamente com relação a $\delta$ e a $\beta$ simultaneamente. Dessa forma, $\delta$ é atualizado em cada um dos dois passos. A razão pela qual isso leva a ganhos de eficiência pode ser explicada a seguir. Em geral, os valores $\left\{T^{*}\right\}$ obtidos na primeira iteração estarão bem próximos aos valores $\{\hat{T}\}$ que correspondem ao mínimo global (ao menos que o valor inicial de $\beta$ esteja muito distante do seu verdadeiro valor $\beta^{0}$ ). Intuitivamente isso ocorre porque uma má-especificação no valor inicial de $\beta$ tem um pequeno efeito sobre as estimativas $\left\{T^{*}\right\}$, uma vez que as últimas dependem majoritariamente nas mudanças nos coeficientes $\delta$ (associadas com as variáveis $z_{t}$ ) entre os regimes.

Considere um segundo passo que consiste em aplicar a regressão de MQO $Y=X \beta+\bar{Z}^{*} \delta+U, \operatorname{com} \bar{Z}^{*}$, partição diagonal de $Z$ na partição $m\{T *\}=\left(T_{1}^{*}, \ldots, T_{m}^{*}\right)$. Se os valores $\left\{T^{*}\right\}$ forem iguais a $\{\hat{T}\}$, correspondendo ao mínimo global, a estimativa de $\beta$ e de $\delta$ a partir desse segundo passo são aquelas correspondendo ao mínimo global. De acordo com Bai \& Perron (2003), experimentos com dados reais e simulados mostraram que, na maioria dos casos, uma única iteração é suficiente.

Para ilustrar o contraste entre os dois métodos acima, considere o que aconteceria se $\delta$ não for re-atualizado no segundo passo. Esse passo se torna uma regressão de MQO simples com a forma $Y-\bar{Z}^{*} \delta^{*}=X \beta+U$. Note que mesmo se $\{T *\}$ for igual a $\{\hat{T}\}$, correspondendo ao mínimo global, a estimativa de $\beta$ não necessariamente estará próxima de $\hat{\beta}$ (o valor no mínimo global), ao menos que $\delta^{*}$ já esteja próximo de $\hat{\delta}$ na primeira iteração (o que pode acontecer apenas com uma pequena probabilidade). Dessa forma, necessita-se de iterações adicionais; e, 
segundo Bai \& Perron (2003), experimentos com dados reais e simulados mostraram que o número de simulações necessárias pode ser grande, mesmo em modelos simples.

O critério de convergência adotado é que a mudança na função objetiva $S_{T}\left(T_{1}, \ldots, T_{m}\right)$ seja menor do que algum $\varepsilon$ arbitrário. Usando o método iterativo sugerido, é possível especificar $\varepsilon=0$ devido à natureza discreta das variáveis $\left(T_{1}, \ldots, T_{m}\right)$. Segundo Bai \& Perron (2003), na maioria dos experimentos realizados, o mínimo foi alcançado após a primeira iteração e a segunda mostrou que não houve nenhuma mudança efetiva na função objetiva.

A eficiência do método proposto acima para alcançar um mínimo global depende de uma escolha apropriada do valor inicial do vetor $\beta$ para começar a iteração. Bai \& Perron (2003) sugerem o seguinte procedimento. Em primeiro lugar, aplicamos o algoritmo de programação dinâmica tratando todos os coeficientes como sujeitos a mudanças, ou seja, tratamos o modelo como um de mudança estrutural pura dado por

$$
y_{t}=x_{t}^{\prime} \delta_{1, j}+z_{t}^{\prime} \delta_{2, j}+u_{t}, t=T_{j-1}+1, \ldots, T_{j},
$$

para $j=1, \ldots, m+1$. A aplicação do algoritmo de programação dinâmica fornece as estimativas $\left(\delta_{1 j}^{a}, \delta_{2 j}^{a} ; j=1, \ldots, m+1\right)$ e $\left(T_{1}^{a}, \ldots, T_{m}^{a}\right)$. Para obter um valor inicial do vetor $\beta$, precisamos apenas usar a regressão de MQO $Y-\bar{Z}^{a} \delta_{2}^{a}=X \beta+U$, em que $\bar{Z}^{a}$ é a partição diagonal de $\mathrm{Z}$ na partição $m\left(T_{1}^{a}, \ldots, T_{m}^{a}\right)$ e $\delta_{2}^{a}$ é a estimativa de $\delta_{2}$. A estimativa obtida, $\beta^{a}$, é usada para iniciar o procedimento de iteração.

O uso desse método para escolher o valor inicial de $\beta$ é justificado pelo fato de que as estimativas $\lambda_{j}^{a}=T_{j}^{a} / T$ das frações de quebras $\lambda_{j}^{0}=T_{j}^{0} / T$ são convergentes à razão $T$ mesmo quando alguns coeficientes não mudam entre os regimes (ver Bai \& Perron (1998)). É preciso apenas que ao menos um coeficiente mude em cada data de quebra. Dessa forma, a estimativa $\beta^{a}$ obtida é assintoticamente equivalente à estimativa $\hat{\beta}$ associada com o mínimo global. Isso 
permite atingir o mínimo global com muito poucas iterações e reduz o risco de atingirmos um mínimo local.

Resultado 3.1: Sejam $\hat{\lambda}=\left(\hat{\lambda}_{1}, \ldots, \hat{\lambda}_{m}\right)=\left(\hat{T}_{1} / T, \ldots, \hat{T}_{m} / T\right)$ as frações de quebras com os correspondentes verdadeiros valores $\lambda^{0}=\left(\lambda_{1}^{0}, \ldots ., \lambda_{m}^{0}\right)$. Sob algumas hipóteses, Bai \& Perron (1998) mostram que $\hat{\lambda}_{k} \stackrel{P}{\rightarrow} \lambda_{k}^{0}, k=1, \ldots, m$. Sejam $\hat{\theta}=(\hat{\beta}, \hat{\delta})$ e $\theta^{0}=\left(\beta^{0}, \delta^{0}\right)$. Sob algumas hipóteses (ver Bai \& Perron (1998)), $\sqrt{T}\left(\hat{\theta}-\theta^{0}\right) \stackrel{d}{\rightarrow} N\left(0, V^{-1} \Phi V^{-1}\right)$ com $V=p \lim T^{-1} \bar{W}^{0} \bar{W}^{0}, \Phi=p \lim T^{-1} \bar{W}^{0} \Omega \bar{W}^{0}$, e $\Omega=E\left(U U^{\prime}\right)$.

Quando os erros forem não-correlacionados e homocedásticos temos $\Phi=\sigma^{2} V$ e a covariância assintótica se reduz a $\sigma^{2} V^{-1}$, que pode ser consistentemente estimada usando uma estimativa consistente de $\sigma^{2}$. Quando temos correlação serial e/ou heterocedasticidade, segundo Bai \& Perron (1998), uma estimativa consistente de $\Phi$ pode ser construída usando a sugestão de Andrews (1991), assumindo distribuições idênticas entre os segmentos ou permitindo que a distribuição de ambos os regressores difiram da distribuição dos erros.

Vamos ver agora um teste de nenhuma quebra estrutural contra a hipótese alternativa de algum número fixo de quebras.

\subsection{Testes para Verificar a Existência de Quebras Estruturais}

\subsubsection{Teste de Nenhuma Quebra}

Bai \& Perron (2003) apresentam um teste denominado $\operatorname{supF}$ de que não há quebra estrutural $(m=0)$ contra a hipótese alternativa de $m=k$ quebras. Seja $\left(T_{1}, \ldots, T_{k}\right)$ uma partição tal que $T_{i}=\left[T \lambda_{i}\right](i=1, \ldots, k)$. Seja $R$ a matriz tal que $(R \delta)^{\prime}=\left(\delta_{1}^{\prime}-\delta_{2}^{\prime}, \ldots, \delta_{k}^{\prime}-\delta_{k+1}^{\prime}\right)$. Bai \& Perron (2003) definem a estatística 


$$
F_{T}\left(\lambda_{1}, \ldots, \lambda_{k} ; q\right)=\frac{1}{T}\left(\frac{T-(k+1) q-p}{k q}\right) \hat{\delta}^{\prime} R^{\prime}\left(R \hat{V}(\hat{\delta}) R^{\prime}\right)^{-1} R \hat{\delta}
$$

em que $\hat{V}(\hat{\delta})$ é uma estimativa da matriz de covariâncias de $\hat{\delta}$, que é robusta a correlação serial e heterocedasticidade, ou seja, uma estimativa consistente de

$$
V(\hat{\delta})=p \lim T\left(\bar{Z}^{\prime} M_{X} \bar{Z}\right)^{-1} \bar{Z}^{\prime} M_{X} \Omega M_{X} \bar{Z}\left(\bar{Z}^{\prime} M_{X} \bar{Z}\right)^{-1} .
$$

Segundo Andrews (1993), a estatística do teste é

$$
\sup F_{T}(k ; q)=F_{T}\left(\hat{\lambda}_{1}, \ldots, \hat{\lambda}_{k} ; q\right),
$$

em que $\hat{\lambda}_{1}, \ldots, \hat{\lambda}_{k}$ minimiza a soma global dos quadrados dos resíduos que é equivalente a maximizar o teste $F$ assumindo erros esféricos. Isso é assintoticamente equivalente a maximizar o teste (3.6), uma vez que as datas de quebra são consistentes mesmo na presença de correlação serial. A distribuição assintótica depende do parâmetro $\varepsilon=h / T$, em que $h$ é o comprimento mínimo de um segmento.

Várias versões do teste podem ser obtidas dependendo das hipóteses feitas com relação à distribuição dos dados e da variabilidade entre os segmentos. Elas estão relacionadas a diferentes especificações na construção da estimativa de $V(\hat{\delta})$ dada por (3.7) (para mais detalhes, ver Bai \& Perron (2003)).

\subsubsection{Teste de L X L+1 Quebras Estruturais (Bai \& Perron (1998))}

Este teste consiste na hipótese nula de $l$ quebras contra a alternativa de que uma quebra adicional exista. Para o modelo com $l$ quebras, os pontos de quebras estruturais estimados $\hat{T}_{1}, \ldots, \hat{T}_{l}$ são obtidos através de uma minimização global da soma dos quadrados dos resíduos.

Bai \& Perron (1998) propõem testar cada segmento $(l+1)$ (obtido usando a partição $\hat{T}_{1}, \ldots, \hat{T}_{l}$ ) para a presença de uma quebra adicional. Assumindo que o tamanho das mudanças é fixa, o teste consiste na aplicação de $(l+1)$ testes da 
hipótese nula de não-mudança estrutural contra a alternativa de uma única mudança. O teste é aplicado para cada segmento contendo as observações $\hat{T}_{i-1}+1$ a $\hat{T}_{i}(i=1, \ldots, l+1), \operatorname{com} \hat{T}_{0}=0$ e $\hat{T}_{l+1}=T$.

Rejeitamos a hipótese nula em favor de um modelo com $(l+1)$ quebras se o valor geral mínimo da soma dos quadrados dos resíduos (sobre todos os segmentos onde uma quebra adicional está inclusa) é suficientemente menor do que a soma dos quadrados dos resíduos do modelo com $l$ quebras. Dessa forma, o momento da quebra selecionado é aquele que possui o valor mínimo geral. Mais precisamente, o teste é definido como

$$
F_{T}(l+1 / l)=\left\{S_{T}\left(\hat{T}_{1}, \ldots, \hat{T}_{l}\right)-\min _{1 \leq i \leq l+1} \inf _{\tau \in \Lambda_{i, \eta}} S_{T}\left(\hat{T}_{1}, \ldots, \hat{T}_{i-1}, \tau, \hat{T}_{i}, \ldots, \hat{T}_{l}\right)\right\} / \hat{\sigma}^{2}
$$

em que

$$
\Lambda_{i, \eta}=\left\{\tau ; \hat{T}_{i-1}+\left(\hat{T}_{i}-\hat{T}_{i-1}\right) \eta \leq \tau \leq \hat{T}_{i}-\left(\hat{T}_{i}-\hat{T}_{i-1}\right) \eta\right\}
$$

e $\hat{\sigma}^{2}$ é uma estimativa consistente de $\sigma^{2}$, sob a hipótese nula. Note que para $i=1, S_{T}\left(\hat{T}_{1}, \ldots, \hat{T}_{i-1}, \tau, \hat{T}_{i}, \ldots, \hat{T}_{l}\right)$ é entendida como $S_{T}\left(\tau, \hat{T}_{1}, \ldots, \hat{T}_{l}\right)$ e para $i=l+1$ como $S_{T}\left(\hat{T}_{1}, \ldots, \hat{T}_{l}, \tau\right)$. Bai \& Perron (1998) derivam valores críticos e uma distribuição $G_{q, \eta}(x)$ para diferentes valores de $l$.

\subsection{Modelo STOPBREAK}

O processo de quebras estocásticas permanentes (STOPBREAK), de Engle \& Smith (1999), foi motivado por uma classe de propriedades que levam em conta quebras estruturais aleatórias em intervalos aleatórios. A análise de uma quebra estrutural, quando o ponto de quebra é conhecido a priori, geralmente envolve 
estatísticas de teste e estimadores padrões, tais como o teste de mudança estrutural de Chow (1960). Porém, quando o ponto de quebra é desconhecido, o problema se torna mais complicado, pois esse ponto de mudança deve ser estimado e, sob a hipótese nula de que não há quebra, esse parâmetro se torna não-identificado. Andrews et al. (1996), Andrews (1993), Hansen (1992), Christiano (1992) e outros, estudaram esse problema em vários contextos.

Quando consideramos múltiplos pontos de quebra, o problema se torna ainda mais complicado porque requer a especificação do número de quebras e a inclusão de parâmetros suficientes para levar em conta cada regime. Isso se torna nãotratável quando o número de pontos de quebra aumenta.

Engle \& Smith (1999) introduziram o modelo STOPBREAK, que trata o problema de quebras estruturais de maneira endógena ao inferir suas magnitudes e freqüências a partir de observações de uma única variável aleatória. Isso permite que o modelo tenha flexibilidade suficiente para reagir a várias quebras sem sobrecarregar os parâmetros.

Definição 3.1: Na sua forma mais simples, o processo STOPBREAK de Engle \& Smith (1999) é dado por:

$$
y_{t}=m_{t}+\varepsilon_{t}, \quad t=0,1, \ldots, T,
$$

em que $\varepsilon_{t}$ é uma seqüência de diferenças martingais estacionária, $\operatorname{com} E\left(\varepsilon_{t}\right)=0$, e $m_{t}$ é a média condicional de $y_{1}$, que varia com o tempo. Assumimos que $m_{0}$ é fixo e conhecido e que $\varepsilon_{0}$ é conhecido. Seja $\left\{\Im_{t}\right\}$ a sigma-álgebra gerada por $\left\{y_{t}, y_{t-1}, \ldots\right\}$ e seja $q_{t}=q\left(\varepsilon_{t}\right)$ a função de transição do processo; dessa forma, $m_{t}=E\left(y_{t} / \mathfrak{I}_{t-1}\right)$ é a média condicional do processo em $t-1$. Assim, $m$, é atualizada por

$$
m_{t}=m_{t-1}+q_{t-1} \varepsilon_{t-1}=m_{0}+\sum_{i=1}^{t} q_{t-i} \varepsilon_{t-i}, \quad t=1,2, \ldots, T
$$


Se a função de transição $q_{t}=q\left(\varepsilon_{t}\right)$ satisfizer $E\left(q_{1} \varepsilon_{t} / \Im_{t-1}\right)=0$, então $m$, também será a previsão de vários períodos de $y_{t}, E\left(y_{t+k} / \Im_{t}\right)$ para $k=1,2, \ldots$ Além disso, assumimos que a função $q_{t}=q\left(\varepsilon_{t}\right)$ é limitada entre zero e um e que $\left.\frac{\partial q}{\partial \varepsilon}\right|_{\varepsilon_{t}}$ é nãonegativa e finita com probabilidade 1. Essa formulação se baseia na hipótese de que as séries temporais possuem menos probabilidade de reverter à média após um grande choque do que depois de um pequeno.

Se $q_{t}=1$, então o processo realizado no tempo $t$ é um passeio aleatório, com quebras estruturais em todos os períodos. Se $q_{t}=0$ (ou caso $E\left(q_{t} \varepsilon_{t} / \mathfrak{I}_{t-1}\right)=0$, como vimos antes), a média condicional é constante e, conseqüentemente, a previsão de longo-prazo para $y_{1}$ também não varia.

A partir das equações (3.11) e (3.12), temos um processo em que a permanência de um choque é determinada endogenamente, a partir da formulação da função de transição $q_{t}$. Por exemplo, no mercado de ações, os investidores podem perceber grandes choques contendo informação com conteúdo significativo e pequenos choques como apenas ruídos. Conseqüentemente, suas expectativas reagem apenas a grandes choques. Uma economia pode estar sujeita a choques permanentes de oferta e choques freqüentes transitórios de demanda.

Nas equações (3.11) e (3.12), q é formulado como uma função apenas do choque corrente; entretanto, qualquer fator que faz parte do conjunto de informação pode ser um argumento na função $q$. Por exemplo, para modelar preços de ações, podemos incluir taxas de juros e de câmbio como variáveis relevantes (ver Engle \& Smith (1999)). Entretanto, é improvável que seja possível levar em conta todos os fatores potenciais que possam causar uma quebra estrutural e, mesmo se isso fosse possível, teríamos uma sobrecarga de parâmetros no processo.

Analogamente ao procedimento adotado por Engle \& Smith (1999), vamos assumir que somente mudanças permanentes irão ser refletidas apenas em um 
choque que é maior do que a regra. Um modelo especificado dessa forma pode não captar pequenas mudanças na média e, conseqüentemente, cometer erros sistemáticos até que a média mude suficientemente mais do que a regra. Esse modelo também tenderá a super reagir a grandes choques transitórios. Entretanto, a simplicidade e flexibilidade desse processo mais do que compensam esses defeitos, se o objetivo for obter boas previsões condicionais (ver Engle \& Smith (1999)).

O modelo STOPBREAK pode também ser considerado como um tipo de mecanismo de correção de erros no sentido de que é mais reacionário do que antecipatório, isto é, ele não procura prever quando uma inovação permanente irá ocorrer, mas sim reagir a choques ao prever seu grau de permanência. Vamos verificar algumas propriedades do processo STOPBREAK.

A característica peculiar do processo STOPBREAK é que o efeito permanente dos choques varia com o tempo e é estocástico. Em alguns períodos as quebras são permanentes enquanto em outros não.

Definição 3.2: A previsão $k$ períodos à frente $f\left(y_{t}, k\right)$ do processo STOPBREAK, dado por (3.11) e (3.12), é

$$
f\left(y_{t}, k\right) \equiv E\left(y_{t+k} / \Im_{t}\right)=m_{t+1} \equiv m_{t}+q_{t} \varepsilon_{t},
$$

uma vez que $f\left(y_{t}, k\right) \equiv E\left(m_{t+k} / \mathfrak{I}_{t}\right)=m_{t+1}$.

Definição 3.3: Considere o processo estocástico $\left\{y_{1}\right\} t=0,1, \ldots$, T. O efeito permanente do choque $t$ é dado por

$$
\lambda_{t} \stackrel{d}{=} \lim _{k \rightarrow \infty} \frac{\partial f\left(y_{t}, k\right)}{\partial \varepsilon_{t}},
$$

em que $f\left(y_{t}, k\right) \equiv E\left(\mathrm{y}_{\mathrm{t}+\mathrm{k}} / \mathfrak{I}_{t}\right), \quad \varepsilon_{t}=y_{t}-E\left(y_{t} / \mathfrak{\Im}_{t-1}\right)=y_{t}-m_{t-1}, \mathfrak{I}_{t}$ incorpora a história presente e passada de $\left\{y_{t}\right\}$ e $\stackrel{d}{=}$ significa igualdade em distribuição. Essa derivada pode ser interpretada como a derivada em relação a $\varepsilon_{1}$, mantendo todo o passado de $y_{t}$ fixo. 
Um martingal possui a propriedade de que todos os choques possuem um efeito permanente, ou seja, $\lambda_{t}=1$ com probabilidade 1 para todo $t$. Inversamente, se $\lambda_{t}=0$ com probabilidade 1 para todo $t$, o processo não possui quebras permanentes. Assim, para $0<\lambda_{t}<1$, temos um processo redutor de quebras, de forma que apenas uma fração do choque permanece. Um exemplo disso é um processo integrado com um componente de média-móvel negativo e invertível. Se $\lambda_{t}<0$, temos quebras permanentes negativas, em que o processo se auto-corrige para os choques e, se $\lambda_{t}>1$, os choques são positivamente ampliados, ou seja, o efeito permanente dos choques é maior do que o efeito inicial. Um processo linear com primeira diferença positivamente correlacionada teria $\lambda_{t}>1$ com probabilidade 1, para todo $t$. Portanto, uma quebra estrutural ocorre no tempo $t$ se o impacto permanente observado ocorrido em $t$ for diferente de zero, isto é, $\lambda_{t} \neq 0$.

Considerando o processo STOPBREAK e diferenciando $f\left(y_{t}, k\right)$ com relação a $\varepsilon_{t}$, obtemos

$$
\frac{\partial f\left(y_{t} ; k\right)}{\partial \varepsilon_{t}} \stackrel{d}{=} q_{t}+\left.\frac{\partial q}{\partial \varepsilon}\right|_{\varepsilon_{t}} \varepsilon_{t}=q_{\imath}\left(1+\eta_{q, t}\right)
$$

em que $\eta_{q, t} \equiv\left(\partial q /\left.\partial \varepsilon\right|_{\varepsilon_{t}}\right)\left(\varepsilon_{\imath} / q_{t}\right)$. Como $q_{t}$ e $\eta_{q, t}$ são não-negativos com probabilidade 1 , temos que $\lambda_{t} \geq 0$ com probabilidade 1 para todo $t$. No processo STOPBREAK, o efeito permanente dos choques varia com o tempo: o valor limite de $\lambda_{t}$ é estocástico.

Esse fato não ocorre, por exemplo, com um processo auto-regressivo estacionário em que os coeficientes mudam de valor, isto é, $y_{t}=\rho_{t} y_{t-1}+\varepsilon_{t}$, em que $\varepsilon_{t}$ é i.i.d. com média zero e $\rho_{t}$ é uma variável aleatória tomando os valores $\rho_{1}$ e $\rho_{2}$, cada um com probabilidade positiva. O mecanismo que determina $\rho_{t}$ pode, por exemplo, ser governado por uma Cadeia de Markov (Hamilton (1989)) ou por um valor limiar (Tong (1983)). Como o processo é estacionário nos dois regimes, $\lambda_{t}=0$ com probabilidade 1 para todo $t$. A taxa pela qual os choques decaem muda 
entre os regimes, mas a série temporal continua estacionária. Isso ocorre porque, assumindo que cada regime é observado com probabilidade positiva, haverá eventualmente períodos suficientes de estacionariedade para o efeito de um choque desaparecer.

Podemos reescrever o STOPBREAK básico das equações (3.11) e (3.12) como

$$
\Delta y_{t}=\varepsilon_{t}-\theta_{t-1} \varepsilon_{t-1}, \quad t=1,2, \ldots, T
$$

em que $\theta_{t-1}=1-q_{t-1}$. Para prever a partir desse processo, é necessário estimar $\varepsilon_{t-1}$, o que requer uma previsão de $\varepsilon_{0}$, que não é observável. Ou seja, é preciso que o processo seja invertível, o que significa que a estimativa de $\varepsilon_{t-1}$ depende de $\varepsilon_{0}$ com um coeficiente que tende a zero com probabilidade 1 para $t$ grande.


previsão de longo-prazo de $y_{t+k}$ cresça primeiro pela proporção de $q_{t}$ e depois ao aumentar o valor de $q_{l}$. Dessa forma, dependendo das propriedades da função $q_{\prime}$, o efeito final de uma mudança em $\varepsilon$, pode exceder o valor da mudança, isto é, os efeitos permanentes dos choques podem ser maiores do que um.

Teorema 3.1 O processo não-linear de média-móvel descrito na equação (3.13) é invertível com probabilidade 1 se $E\left(\left|1-q_{t}\left(1+\eta_{q t}\right)\right| / \mathfrak{J}_{t-1}\right) \leq c_{t} \leq 1$, em que $\eta_{q_{t}}=\left.\left(\varepsilon_{t} / q_{t}\right)\left(\partial q_{t} / \partial \varepsilon_{t}\right)\right|_{\varepsilon_{t}}$ e $\left\{c_{t}\right\}$ é uma seqüência determinística tal que $\lim _{T \rightarrow \infty} \prod_{t=1}^{T} c_{t}=0$.

Demonstração: Ver Engle \& Smith (1999).

O exemplo mais simples de uma forma funcional para a função de transição $q_{1}$, que satisfaz as condições do Teorema 3.1 é uma função limiar, em que $q_{t}$ toma o valor um para valores de $\varepsilon$, maiores, em valores absolutos, do que algum limiar, e zero caso contrário. Embora $\eta_{q t}=\left.\left(\varepsilon_{t} / q_{t}\right)\left(\partial q_{t} / \partial \varepsilon_{t}\right)\right|_{\varepsilon_{t}}$ seja infinita no limiar, podemos ainda supor invertibilidade com probabilidade 1, uma vez que esse evento ocorre em um conjunto de medida zero. 
Engle \& Smith (1999) especificam a função $q_{\text {, }}$ do STOPBREAK como contínua. Isso permite um processo suave de quebras permanentes, de forma que choques considerados intermediários possuem apenas alguma parte que é permanente. A intuição sugere que essa especificação menos rígida se adapta melhor à evidência empírica.

Suponha que um modelo corretamente especificado para $q$, é dado por

$$
q_{t}(\gamma)=\frac{\varepsilon_{t}^{2}}{\gamma+\varepsilon_{t}^{2}}, \quad \gamma>0
$$

Sob essa especificação, há uma transição suave entre os dois extremos, como podemos ver na Figura 3.2.
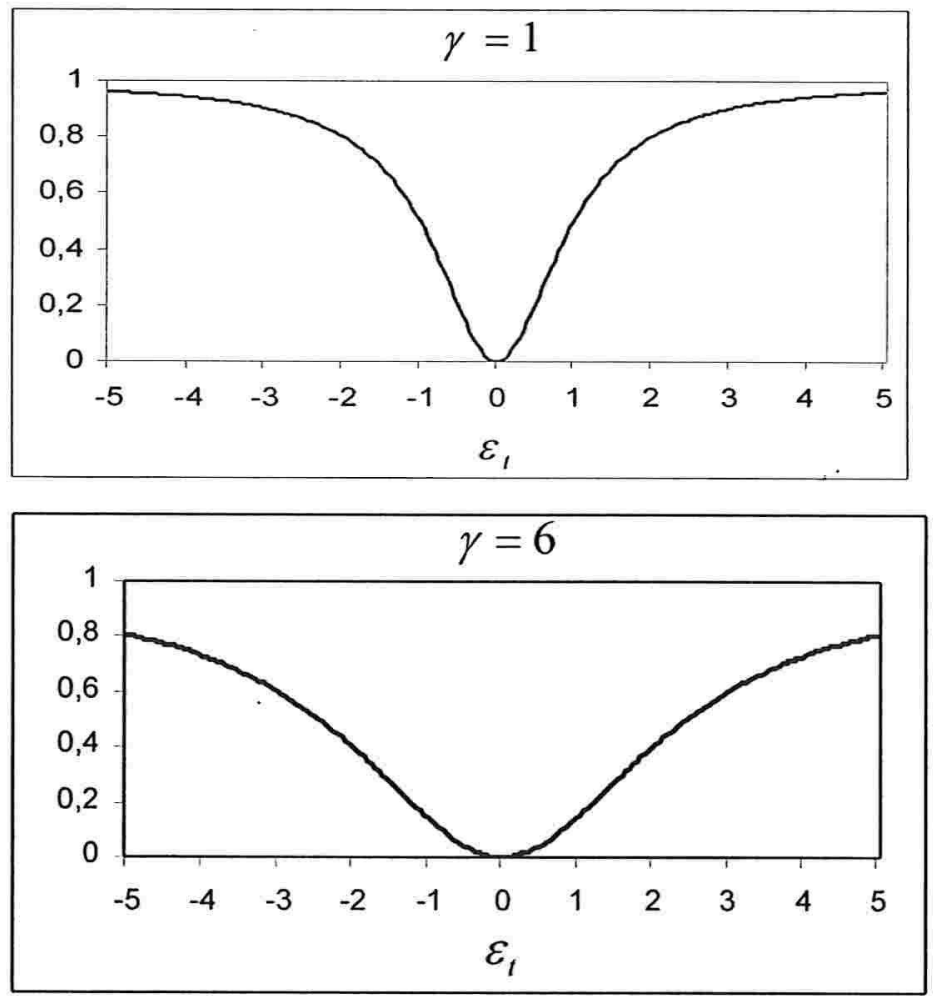

Figura 3.2 - Função $q_{t}(\gamma)$ para $\gamma=1$ e $\gamma=6$ 
Uma função de transição mais rápida é obtida ao aumentar o expoente de $\varepsilon_{t}$ para quatro. A maioria das funções de transição da forma da equação (3.14) satisfaz as condições do Teorema 3.1. Além de ser simples, outra vantagem dessa especificação é que ela tende a um à medida que $\gamma$ tende a zero. Isso permite a realização de um teste paramétrico da hipótese nula de passeio aleatório. Diversas outras especificações, tais como a função de transição logística, pode apenas se transformar nessa forma, caso um parâmetro extra seja adicionado.

Outra propriedade útil da especificação (3.14) é que, para todo $\gamma>0, q_{t}=0$ se e somente se, $\varepsilon_{t}=0$; ou seja, nenhum choque positivo é completamente transitório. Dessa forma, em períodos de pequenos erros, o processo é apenas aproximadamente estacionário. Essa aproximação é benéfica para o teste de hipóteses, pois gera estatísticas de teste com as distribuições bem especificadas (Normal, F-Snedecor, t-Student).

As Figuras 3.3 e 3.4 mostram duas simulações de 1000 observações do processo STOPBREAK descrito por (3.11) e (3.12), com $\varepsilon_{t} \sim N(0,1), q_{t}$ dado por (3.14), para $\gamma=1$ e $\gamma=6$.
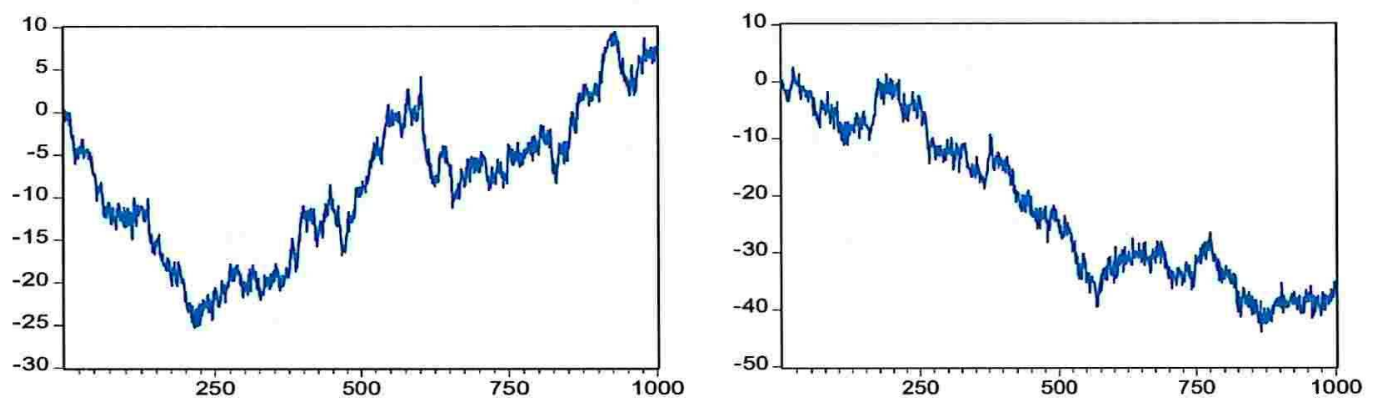

Figura 3.3 - Simulação de dois processos STOPBREAK com $\varepsilon_{t} \sim N(0,1)$ e $q_{t}$ dado por (3.14) $\operatorname{com} \gamma=1$ 

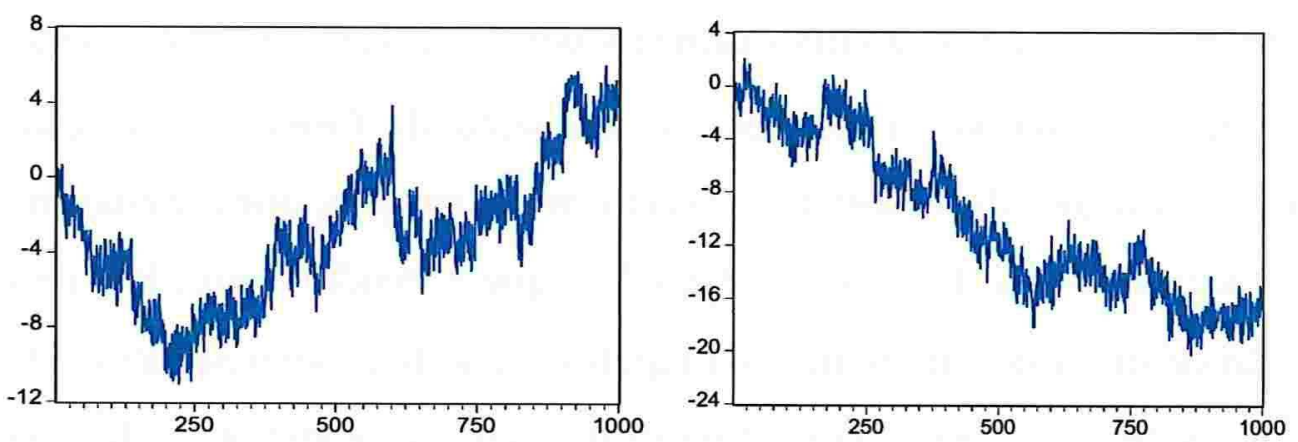

Figura 3.4 - Simulação de dois processos STOPBREAK com $\varepsilon_{t} \sim N(0,1)$ e $q_{t}$ dado por (3.14) com $\gamma=6$

O processo STOPBREAK é um caso especial do seguinte processo geral de quebra:

$$
\begin{aligned}
& A(L) B(L)\left(y_{t}-x_{t}^{\prime} \delta\right)=A(L) z_{t} \varepsilon_{t}+B(L)\left(1-z_{t-1}\right) \varepsilon_{t}, t=1,2, \ldots, T, \\
& A(L)=1-\alpha_{1} L-\alpha_{2} L^{2}-\ldots-\alpha_{p} L^{p}, \\
& B(L)=1-\beta_{1} L-\beta_{2} L^{2}-\ldots-\beta_{s} L^{s},
\end{aligned}
$$

em que $x_{t}$ denota um vetor de variáveis explicativas, $\delta$ um vetor de coeficientes, $\varepsilon_{t}$ um termo de inovação, $z_{t-1}$ alguma função mensurável com informação até $t-1$, e $L$ o operador defasagem.

Quando $z_{t-1}=0$ com probabilidade 1 para todo $t, B(L)$ é um fator autoregressivo e de média-móvel comum que se cancela, deixando $A(L)\left(y_{t}-x_{t}^{\prime} \delta\right)=\varepsilon_{t}$. Similarmente, o processo se reduz para $B(L)\left(y_{t}-x_{t}^{\prime} \delta\right)=\varepsilon_{t}$ quando $z_{t-1}=1$ com probabilidade 1 para todo $t$. Fazendo $\delta=0, B(L)=1-L, A(L)=$ 1, e $z_{t-1}=q_{t-1}\left(\gamma_{0}\right)$, obtemos o processo STOPBREAK básico de Engle \& Smith (1999).

O processo geral descrito na equação (3.15) irá exibir um efeito permanente dos choques apenas se o operador $B(L)$ tiver uma raiz unitária e se os outros operadores tiverem todas as raízes fora do círculo unitário. Isso faz com que 
tenhamos modelos com ou sem choques permanentes ou transitórios. Por exemplo, considere $\delta=0, B(L)=1-L, A(L)=1$ e suponha que $z_{t-1}=1$ com probabilidade 1 se $t=t^{*}$ e $z_{t-1}=0$ com probabilidade 1 se $t \neq t^{*}$, em que $1<t^{*}<T$. Esse processo tem uma quebra em sua média em $t=t^{*}$, isto é, $\lambda_{t}=1$ com probabilidade $1 \mathrm{em}$ $t=t *$ e $\lambda_{t}=0$ com probabilidade 1 em todos os outros períodos.

A formulação geral de (3.15) também revela um número de possíveis generalizações para o STOPBREAK simples. Por exemplo, o processo poderia ter alguma correlação temporal quando está em períodos de "não-quebra". Isso corresponde a $\delta=0, B(L)=1-L, \mathrm{~A}(\mathrm{~L})=1-\alpha_{0} L$, e $z_{t-1}=q_{t-1}\left(\gamma_{0}\right)$, e implica a seguinte representação de "média-móvel":

$$
\Delta y_{t}=\alpha_{0} \Delta y_{t-1}+\varepsilon_{t}-\theta_{t-1} \varepsilon_{t-1}, \quad t=1,2, \ldots, T,
$$

em que $\theta_{t-1}=1-\left(1-\alpha_{0}\right) q_{t-1}$ e $0 \leq \alpha_{0} \leq 1$. Agora $\Delta y_{t}$ tem um AR(1) e um passeio aleatório como seus dois extremos.

A estimação do STOPBREAK de Engle \& Smith (1999) é feita pelo método de Quase-Verossimilhança. Embora a verossimilhança seja construída supondo $\varepsilon_{t} \sim N\left(0, \sigma^{2}\right)$, não é preciso que essa seja a verdadeira distribuição. Dada a invertibilidade, podemos especificar o log da função de verossimilhança condicional para o processo dado na equação (3.16) como

$$
l\left(y^{T}, \varphi\right)=-\frac{1}{2 \sigma^{2}} \sum_{t=1}^{T}\left(\Delta y_{t}-\alpha_{0} \Delta y_{t-1}+\theta_{t-1} \varepsilon_{t-1}\right)^{2}-\frac{T}{2} \log \left(2 \pi \sigma^{2}\right),
$$

em que $\varphi=(\gamma \alpha \sigma)^{\prime}$ e $y^{T}=\left(y_{1}, y_{2}, \ldots, y_{T}\right)$. As funções escore são dadas por

$$
\begin{aligned}
& \frac{\partial l}{\partial \gamma}=-\frac{1}{\sigma^{2}} \sum_{t=1}^{T} \varepsilon_{t} w_{t}, \\
& \frac{\partial l}{\partial \alpha}=-\frac{1}{\sigma^{2}} \sum_{t=1}^{T} \varepsilon_{t} v_{t}, \mathrm{e} \\
& \frac{\partial l}{\partial \sigma^{2}}=-\frac{1}{\sigma^{3}} \sum_{t=1}^{T} \varepsilon_{t}^{2}-T \sigma^{-1},
\end{aligned}
$$

em que 


$$
\begin{aligned}
& w_{t}=b_{t-1} w_{t-1}-(1-\alpha) \frac{\partial q_{t-1}}{\partial \gamma} \varepsilon_{t-1}, \\
& v_{t}=b_{t-1} v_{t-1}+q_{t-1} \varepsilon_{t-1}-\Delta y_{t-1} \\
& b_{t-1}=1-(1-\alpha)\left(1+\eta_{q t-1}\right) q_{t-1},
\end{aligned}
$$

e, uma vez que $\varepsilon_{0}$ é fixo e conhecido, $b_{0}=w_{0}=v_{0}=0$ com probabilidade 1 .

A presença de grandes choques permanentes indicaria que erros leptocúrticos são prováveis. Sob certas condições ${ }^{4}$ em $\varepsilon_{t}$, Engle \& Smith (1999) mostram que o estimador de Quase-Verossimilhança é consistente e tem distribuição assintótica normal.

${ }^{4}$ Essas hipóteses são que $\left\{\varepsilon_{t}, \Im_{t}\right\}$ são seqüências de diferenças martingais estritamente estacionárias e que $E\left|\varepsilon_{l}\right|^{2 p}<\infty$ para algum $p>1$. Para mais detalhes, ver Engle \& Smith (1999). 


\section{Capítulo 4}

\section{Modelos de Mudança de Regime}

\subsection{Introdução}

Muitas séries temporais financeiras possuem a propriedade de mudança de regime estocástica. LeBaron (1992) mostra que as autocorrelações dos retornos de ações estão relacionadas ao nível de volatilidade desses retornos. Particularmente, as autocorrelações tendem a serem maiores em períodos de baixa volatilidade e menores em períodos de alta volatilidade.

Os períodos de alta e baixa volatilidade podem ser interpretados como regimes distintos, ou seja, o nível de volatilidade pode ser considerado como um processo determinante do regime. Vários modelos de séries temporais foram propostos para formalizar a idéia da existência de diferentes regimes gerados por um processo estocástico. Vamos olhar alguns deles, restringindo nossa atenção para modelos que assumem que em cada um dos regimes o comportamento dinâmico das séries temporais pode ser descrito adequadamente por um modelo AR. Generalizações do modelo MA para um contexto de mudança de regime também já foram consideradas (ver de Gooijer (1998)). 


\subsection{Modelos de Regressão Limiar e de Transição Suave}

Um modelo que assume que o regime que ocorre no tempo $t$ pode ser determinado por uma variável $q_{t}$ é o modelo autoregressivo limiar (TAR), do inglês Threshold Autoregressive, proposto inicialmente por Tong (1978). Esse modelo assume que o regime é determinado pelo valor de $q_{t}$ relativo a um valor limiar, denotado por $c$. Um caso especial aparece quando a variável limiar $q_{t}$ é tomada como um valor defasado da série temporal, ou seja, $q_{t}=y_{t-d}$ para um certo inteiro $d>0$. Nesse caso, como o regime é determinado pela própria série temporal, chamamos o modelo resultante de SETAR, do inglês Self-Exciting Threshold Autoregressive.

Por exemplo, quando $d=1$ e um modelo AR(1) é assumido em ambos os regimes, um modelo SETAR de dois regimes é dado por:

$$
y_{t}=\left\{\begin{array}{l}
\phi_{0,1}+\phi_{1,1} y_{t-1}+\varepsilon_{t} \text { se } y_{t-1} \leq c, \\
\phi_{0,2}+\phi_{1,2} y_{t-1}+\varepsilon_{1} \text { se } y_{t-1}>c,
\end{array}\right.
$$

em que $\varepsilon_{t}$ é um ruído branco i.i.d. condicional ao passado da série temporal, denotado por $\Omega_{t-1}=\left\{y_{t-1}, y_{t-2}, \ldots, y_{t-(p-1)}, y_{t-p}\right\}$ como anteriormente, ou seja, $E\left[\varepsilon_{t} / \Omega_{t-1}\right]=0$ e $E\left[\varepsilon_{t}^{2} / \Omega_{t-1}\right]=\sigma^{2}$.

Outra forma de escrever o modelo SETAR é dada por:

$$
y_{t}=\left(\phi_{0,1}+\phi_{1,1} y_{t-1}\right)\left(1-I\left[y_{t-1}>c\right]\right)+\left(\phi_{0,2}+\phi_{1,2} y_{t-1}\right)\left(I\left[y_{t-1}>c\right]\right)+\varepsilon_{t}
$$

em que $I[A]$ é uma função indicadora com $I[A]=1$ se o evento A ocorre e $I[A]=0$ caso contrário.

Na Figura 4.1 temos quatro simulações de 200 observações do modelo SETAR dado por (4.2) com $\varepsilon_{\imath} \sim N(0,1), \phi_{1,1}=-0.5, \phi_{1,2}=0.5$ e $c=0$. 


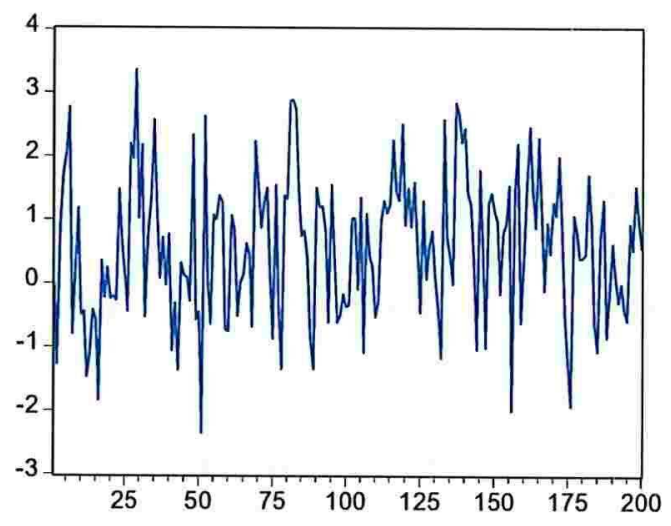

(a) $\phi_{0,1}=0, \phi_{0,2}=0$

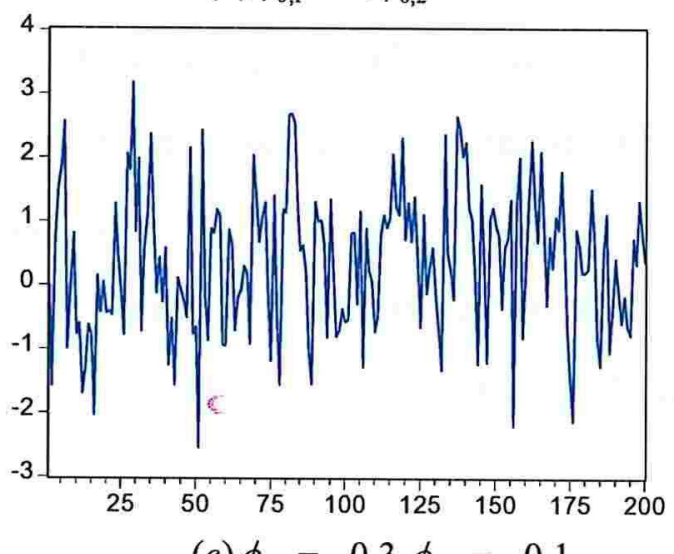

(c) $\phi_{0,1}=-0.3, \phi_{0,2}=-0.1$

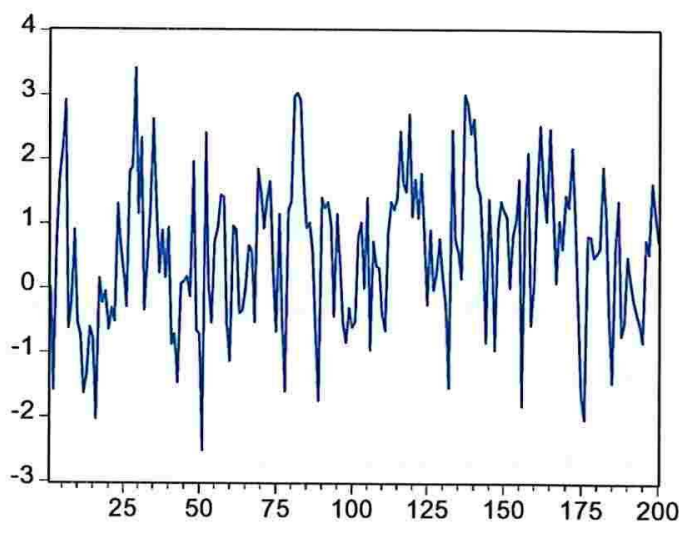

(b) $\phi_{0,1}=-0.3, \phi_{0,2}=0.1$

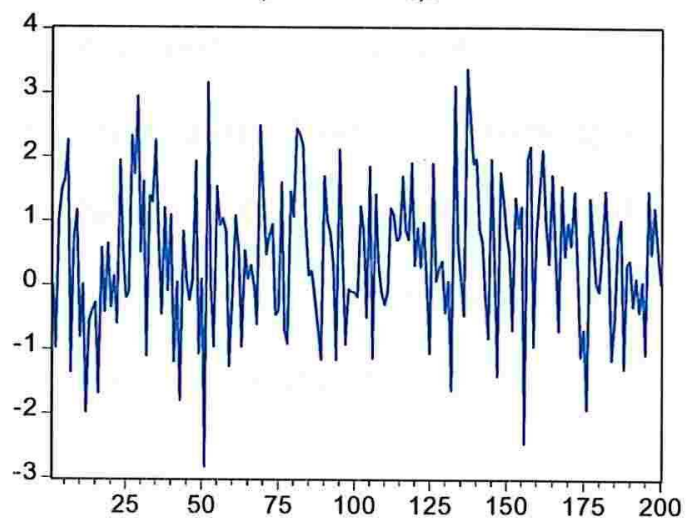

(d) $\phi_{0,1}=0.3, \phi_{0,2}=-0.3$

Figura 4.1 - Simulação de quatro séries geradas a partir do modelo SETAR

O modelo SETAR assume que a fronteira entre os dois regimes é dada por um valor específico da variável limiar $y_{t-1}$. Uma transição gradual entre os diferentes regimes pode ser obtida ao substituirmos a função indicadora $I[A]$ em (4.2) por uma função contínua $G\left(y_{t-1} ; \gamma ; c\right)$, que muda suavemente de 0 a 1 à medida que $y_{t-1}$ aumenta. O modelo resultante é chamado de autoregressão de transição suave, STAR, do inglês Smooth Transition Auto-Regressive e é dado por

$$
y_{t}=\left(\phi_{0,1}+\phi_{1,1} y_{t-1}\right)\left(1-G\left(y_{t-1} ; \gamma, c\right)\right)+\left(\phi_{0,2}+\phi_{1,2} y_{t-1}\right)\left(G\left(y_{t-1} ; \gamma, c\right)\right)+\varepsilon_{t} .
$$

Uma escolha muito usada para a função de transição $G\left(y_{t-1} ; \gamma, c\right)$ é a função logística 


$$
G\left(y_{t-1} ; \gamma, c\right)=\frac{1}{1+\exp \left(-\gamma\left[y_{t-1}-c\right]\right)},
$$

e o modelo resultante é chamado de modelo STAR Logístico (LSTAR). O parâmetro $c$ em (4.4) pode ser interpretado como o limiar entre os dois regimes correspondentes a $G\left(y_{t-1} ; \gamma, c\right)=0$ e $G\left(y_{t-1} ; \gamma, c\right)=1$ no sentido de que a função logística muda monotonicamente de 0 a 1 à medida que $y_{t-1}$ cresce.

O parâmetro $\gamma$ determina a suavidade da mudança no valor da função logística e, dessa forma, a transição de um regime para outro. Como podemos notar na Figura 4.2 abaixo, à medida que $\gamma$ aumenta, a mudança de $G\left(y_{t-1} ; \gamma, c\right)$ de 0 a 1 fica quase instantânea para $y_{t-1}=c$ e, conseqüentemente, a função logística $G\left(y_{t-1} ; \gamma, c\right)$ aproxima a função indicadora $I\left[y_{t-1}>c\right]$.

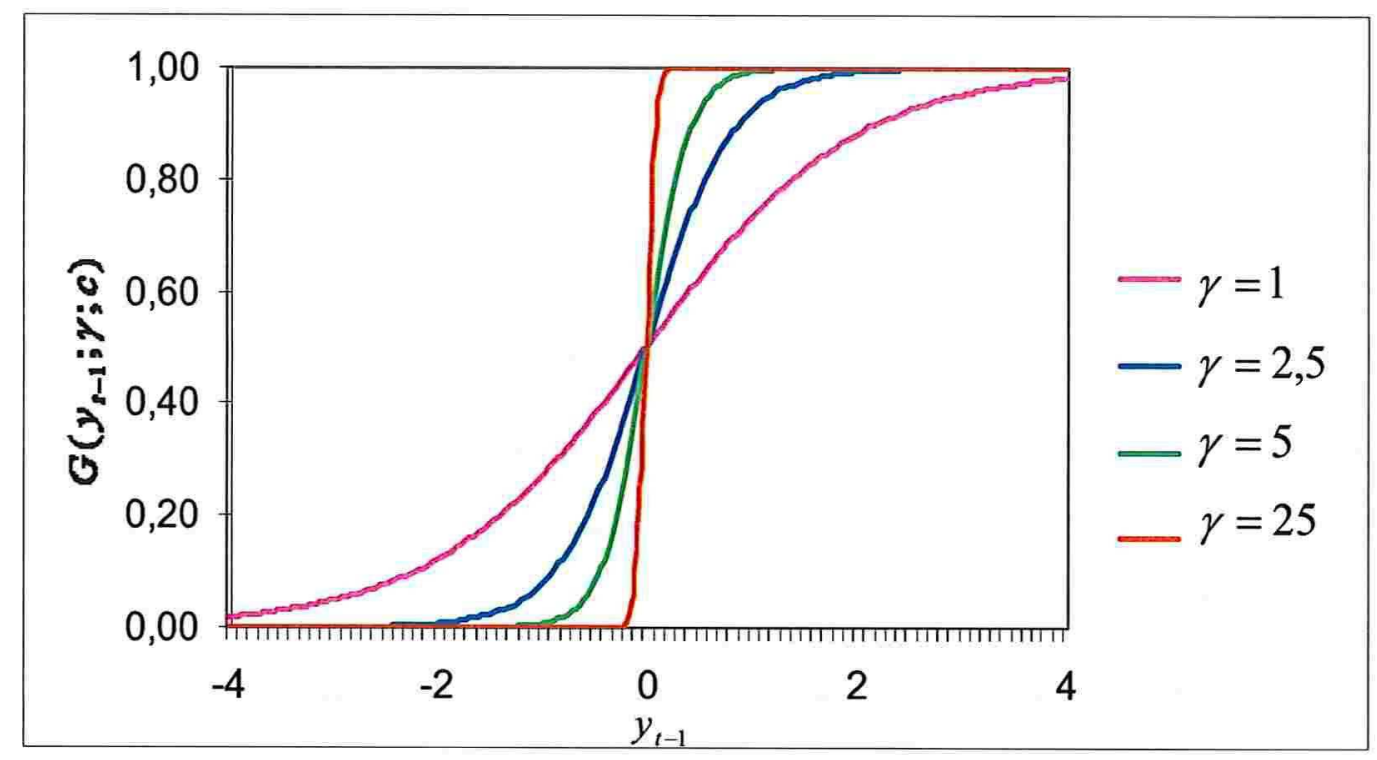

Figura 4.2 - $G\left(y_{t-1} ; \gamma, c\right)$ para vários valores de $\gamma$ e limiar $c=0$

Nas Figuras 4.3 e 4.4 temos duas simulações de 200 observações de um modelo LSTAR dado por (4.3) e (4.4), com parâmetros $\phi_{0,1}=4, \phi_{1,1}=0.8$ para o primeiro 
regime e $\phi_{0,2}=2$ e $\phi_{1,2}=0.05$ para o segundo regime, com $\varepsilon_{t} \sim N(0,1)$ e $c=0$, para $\gamma=1$ e para $\gamma=25$. Note que a mudança de regime é mais abrupta para $\gamma=25$.

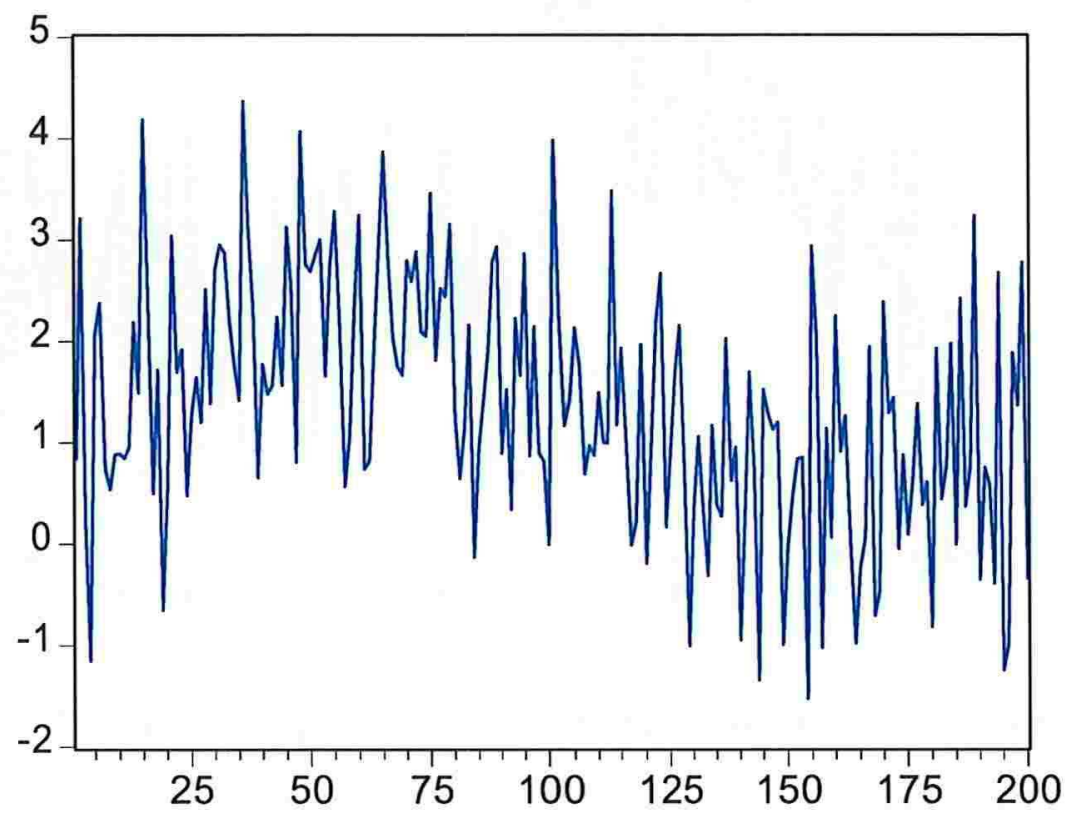

Figura 4.3 - Simulação de um LSTAR com $\phi_{0,1}=4, \phi_{1,1}=0.8, \phi_{0,2}=2, \phi_{1,2}=0.05$,

$$
\varepsilon_{t} \sim N(0,1), \gamma=1 \text { e } c=0
$$




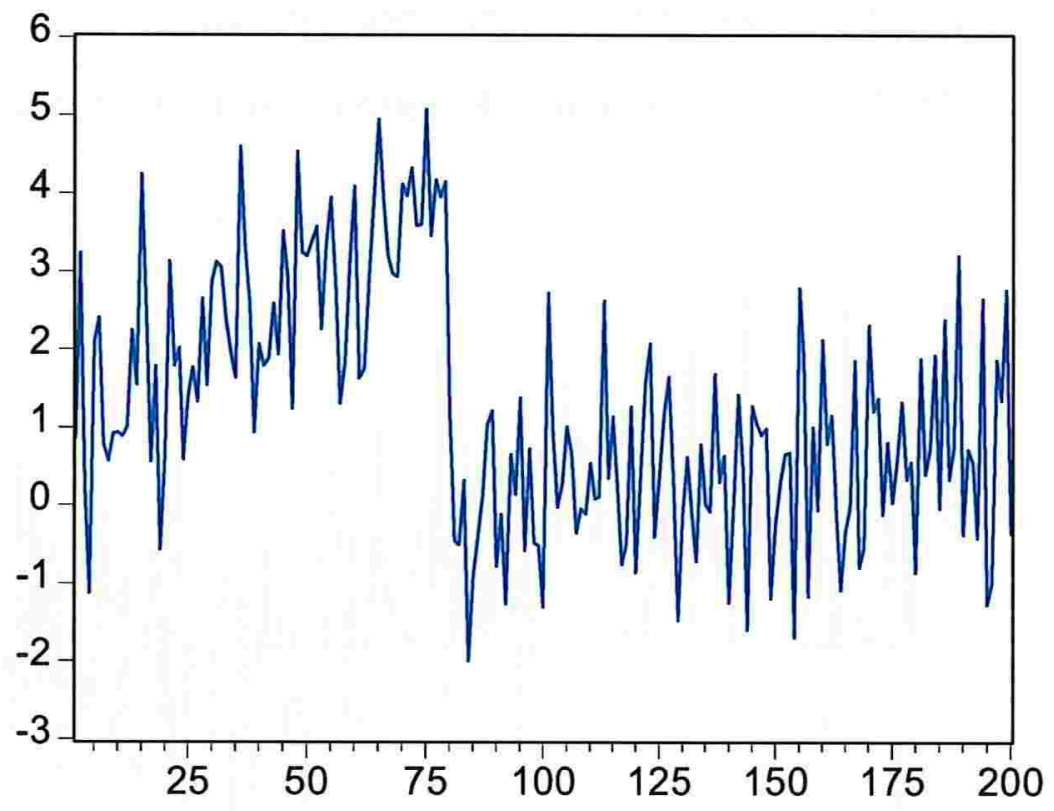

Figura 4.4 - Simulação de um LSTAR com $\phi_{0,1}=4, \phi_{1,1}=0.8, \phi_{0,2}=2$, $\phi_{1,2}=0.05, \varepsilon_{t} \sim N(0,1), \gamma=25$ e $c=0$

Embora os modelos SETAR e STAR com um modelo AR(1) em ambos regimes possam gerar uma grande variedade de padrões de dinâmica, na prática podemos utilizar modelos AR de ordens maiores nos diferentes regimes. Por exemplo, no caso de dois regimes, as ordens AR podem ser ajustadas para $p_{1}$ e $p_{2}$ no primeiro e segundo regime, respectivamente. Nesse caso o modelo SETAR fica na forma

$$
y_{t}=\left\{\begin{array}{l}
\phi_{0,1}+\phi_{1,1} y_{t-1}+\ldots+\phi_{p_{1}, 1} y_{t-p_{1}}+\varepsilon_{t} \text { se } y_{t-1} \leq c \\
\phi_{0,2}+\phi_{1,2} y_{t-1}+\ldots+\phi_{p_{2}, 2} y_{t-p_{2}}+\varepsilon_{t} \text { se } y_{t-1}>c
\end{array}\right.
$$

em que o modelo STAR equivalente é dado por

$$
y_{t}=\left(\phi_{0,1}+\phi_{1,} y_{t-1}+\ldots+\phi_{p_{1},} y_{t-p_{1}}\right)\left(1-G\left(y_{t-1} ; \gamma, c\right)\right)+\left(\phi_{0,2}+\phi_{, 2} y_{t-1}+\ldots+\phi_{p_{2}, 2} y_{t-p_{2}}\right)\left(G\left(y_{t-1} ; \gamma, c\right)\right)+\varepsilon_{t}
$$


A identificação da ordem de defasagem dos modelos auto-regressivos pode ser feita por dois procedimentos. Um deles é começar especificando um modelo linear $\operatorname{AR}(\mathrm{p})$ para $y_{\text {, }}$ e assumir que a ordem $p$, baseada nas autocorrelações (parciais) de $y_{t}$ ou um critério de informação como AIC ou BIC, é a ordem apropriada em ambos os regimes do modelo não-linear. Esse procedimento é perigoso, no sentido de que pode facilmente acontecer que a ordem de defasagem obtida não é apropriada. Além disso, modelos não-lineares relativamente simples podem dar origem a estruturas de autocorrelações complicadas, que podem ser capturadas apenas por um modelo $\mathrm{AR}(\mathrm{p})$ com um $p$ muito grande.

Outro procedimento seria escolher os lags $p_{1}$ e $p_{2}$ em (4.5) e (4.6) baseando-se diretamente em um critério de informação. Sin \& White (1996) demonstram que esse procedimento é consistente, no sentido de que as ordens de defasagem corretas irão ser selecionadas assintoticamente com probabilidade 1 .

Tong (1990) define um AIC alternativo para um modelo SETAR de dois regimes, como a soma dos AIC dos modelos $\mathrm{AR}$ dos dois regimes

$$
\operatorname{AIC}\left(p_{1}, p_{2}\right)=n_{1} \ln \hat{\sigma}_{1}^{2}+n_{2} \ln \hat{\sigma}_{2}^{2}+2\left(p_{1}+1\right)+2\left(p_{2}+1\right)
$$

em que $n_{j}, j=1,2$, é o número de observações no $j$-ésimo regime, e $\hat{\sigma}_{j}^{2}, j=1$, é a variância dos resíduos no $j$-ésimo regime. Embora $\varepsilon_{t}$ tenha a mesma variância para os dois regimes, $\hat{\sigma}_{1}^{2}$ e $\hat{\sigma}_{2}^{2}$ estimadas podem ser diferentes. O BIC para um modelo SETAR pode ser definido analogamente como

$$
B I C\left(p_{1}, p_{2}\right)=n_{1} \ln \hat{\sigma}_{1}^{2}+n_{2} \ln \hat{\sigma}_{2}^{2}+\left(p_{1}+1\right) \ln n_{1}+\left(p_{2}+1\right) \ln n_{2} .
$$

As ordens de defasagem escolhidas nos dois regimes serão aquelas que minimizarem o critério de informação. 
Os parâmetros de estimação de um modelo SETAR podem convenientemente ser estimados por mínimos quadrados condicionais seqüenciais. Sob a hipótese adicional de que $\varepsilon_{t} \sim N(.,$.$) , as estimativas resultantes equivalem às estimativas de$ máxima verossimilhança.

Por simplicidade, vamos discutir o problema de estimação para modelos de dois regimes com ordens autoregressivas iguais nos dois regimes, ou seja, $p_{1}=p_{2}=p$. Re-escrevendo (4.5), temos

$$
y_{t}=\left(\phi_{0,1}+\phi_{1,1} y_{t-1}+\ldots+\phi_{p, 1} y_{t-p}\right) I\left[y_{t-1} \leq c\right]+\left(\phi_{0,2}+\phi_{1,2} y_{t-1}+\ldots+\phi_{p, 2} y_{t-p}\right) I\left[y_{t-1}>c\right]+\varepsilon_{t},
$$

ou, mais resumidamente, como

$$
y_{t}=\phi_{1}^{\prime} x_{t} I\left[y_{t-1} \leq c\right]+\phi_{2}^{\prime} x_{t} I\left[y_{t-1}>c\right]+\varepsilon_{t},
$$

em que $\phi_{j}=\left(\phi_{0, j}, \phi_{1, j}, \ldots, \phi_{p, j}\right)^{\prime}, j=1,2$, e $x_{t}=\left(1, y_{t-1}, \ldots, y_{t-p}\right)^{\prime}$. Como podemos observar, quando o limiar $c$ é fixo, o modelo é linear em todos os outros parâmetros.

Os estimadores de $\phi=\left(\phi_{1}^{\prime}, \phi_{2}^{\prime}\right)^{\prime}$ são facilmente obtidos por MQO por

$$
\hat{\phi}(c)=\left(\sum_{t=1}^{n} x_{t}(c) x_{t}(c)^{\prime}\right)^{-1}\left(\sum_{t=1}^{n} x_{t}(c) y_{t}\right),
$$

em que $x_{t}(c)=\left(x_{t}^{\prime} I\left[y_{t-1} \leq c\right], x_{t}^{\prime} I\left[y_{t-1}>c\right]\right)^{\prime}$ e a notação $\hat{\phi}(c)$ é usada para indicar que a estimativa de $\phi$ é condicional ao valor de $c$. Os resíduos correspondentes são denotados por $\hat{\varepsilon}_{t}(c)=y_{t}-\hat{\phi}(c) x_{t}(c)$ com variância $\hat{\sigma}^{2}(c)=\frac{1}{n} \sum_{t=1}^{n} \hat{\varepsilon}_{t}(c)^{2}$.

O estimador de mínimos quadrados de $c$ pode ser obtido ao minimizarmos essa variância dos resíduos, obtendo

$$
c=\underset{c \in C}{\arg \min } \hat{\sigma}^{2}(c)
$$

em que $\mathrm{C}$ denota o conjunto de todos valores possíveis. Os estimadores finais dos parâmetros autoregressivos são dados por $\hat{\phi}=\hat{\phi}(c)$, enquanto que a variância dos resíduos é estimada por $\hat{\sigma}^{2}=\hat{\sigma}^{2}(\hat{c})$.

O problema de minimização de (4.12) pode ser resolvido através de busca direta. Basta computar a variância dos resíduos $\hat{\sigma}(c)$ apenas para valores limiares 
iguais às estatísticas de ordem de $y_{t-1}$, ou seja, para $c=y_{(i)}$ para cada $i$ tal que $y_{(i)} \in C$. Isso decorre do fato de que o valor de $\hat{\sigma}(c)$ não muda quando $c$ varia entre duas estatísticas de ordem consecutivas, uma vez que nenhuma observação muda de um regime para outro nesse caso.

Chan (1993) demonstra que o estimador de mínimos quadrados do limiar $\hat{c}$ é $\sqrt{n}$-consistente e assintoticamente independente das outras estimativas dos parâmetros.

Até aqui assumimos implicitamente que a variável limiar $q_{t}$, que define o regime que ocorre em qualquer ponto no tempo, é conhecida (e igual a $y_{t-1}$ ). $\mathrm{Na}$ prática, a variável limiar apropriada é desconhecida e uma importante questão é como ela pode ser determinada. No contexto de modelos SETAR, devemos restringir nossa atenção para variáveis endógenas defasadas $y_{t-d}$, para inteiros positivos $d$, como variáveis limiares candidatas. Nesse caso, $d$ pode ser estimada junto com os outros parâmetros do modelo, ao realizarmos os cálculos acima para diversas escolhas de $d\left(d \in\left\{1, \ldots, d^{*}\right\}\right.$ para algum limite superior $\left.d^{*}\right)$, e estimando $d$ como o valor que minimiza a variância dos resíduos.

Uma maneira alternativa de interpretar esse procedimento é que o grid search em (4.12) é aumentado com uma busca sobre $d$, isto é, o problema de minimização se torna

$$
(\hat{c}, \hat{d})=\underset{c \in C, d \in D}{\arg \min } \hat{\sigma}^{2}(c, d),
$$

em que $D=\left\{1, \ldots, d^{*}\right\}$ e a notação $\hat{\sigma}^{2}(c, d)$ é usada para indicar que o estimador da variância dos resíduos depende agora tanto de $d$ como de $c$. Como o espaço paramétrico de $d$ é discreto, o estimador de mínimos quadrados $\hat{d}$ é consistente e $d$ pode ser tratado como conhecido quando computarmos intervalos de confiança para os parâmetros restantes, por exemplo.

Um procedimento similar é feito quando usamos uma variável limiar exógena $q_{1}$. Nesse caso, o modelo SETAR é estimado com diferentes candidatos de 
variáveis limiares, e a variável que obtiver o melhor ajuste será selecionada. Para mais detalhes do método de selecionar a variável limiar, ver Chen (1995).

Nota-se que há um loop na especificação dos modelos SETAR. Para determinar as ordens apropriadas dos modelos AR nos dois regimes com, por exemplo, o AIC dado em (4.7), a variável limiar foi dada como conhecida, enquanto que para determinar a variável limiar apropriada usando o grid search descrito acima, as ordens autoregressivas foram tomadas como conhecidas. Uma maneira de quebrar esse loop seria incluir a procura pela variável limiar apropriada na minimização do critério de informação, ou seja, minimizar o AIC em relação a $p_{1}, p_{2}$ e $d$ como foi sugerido por Tong (1990).

A estimação dos parâmetros do modelo STAR dado em (4.6), em que a variável de transição é $y_{t-1}$, é uma aplicação direta do método de mínimos quadrados não-lineares, ou seja, o vetor de parâmetros $\theta=\left(\phi_{1}^{\prime}, \phi_{2}^{\prime}, \gamma, c\right)^{\prime}$ pode ser estimado como

$$
\hat{\theta}=\underset{\theta}{\arg \min } \sum_{t=1}^{n}\left[y_{t}-F\left(x_{t} ; \theta\right)\right]^{2},
$$

em que $F\left(x_{i} ; \theta\right)$ é a estrutura do modelo, ou seja,

$$
F\left(x_{i} ; \theta\right)=\dot{\phi_{1}} x_{t}\left(1-G\left(y_{t-1} ; \gamma, c\right)\right)+\dot{\phi_{2}} x_{t} G\left(y_{t-1} ; \gamma, c\right) \text {. }
$$

Sob a hipótese adicional de que os erros $\varepsilon_{l}$ são normalmente distribuídos, o método de mínimos quadrados não-lineares equivale ao método de máxima verossimilhança. Caso contrário, as estimativas de mínimos quadrados nãolineares poderiam ser interpretadas como estimativas de quase-máxima verossimilhança. Sob certas condições de regularidade (ver White \& Domowitz (1984), Gallant (1987)), o estimador de mínimos quadrados não-lineares é consistente e assintoticamente normal, ou seja,

$$
\sqrt{T}\left(\hat{\theta}-\theta_{0}\right) \rightarrow N(0, C),
$$


em que $\theta_{0}$ denota os verdadeiros valores dos parâmetros. A matriz de covariância assintótica $C$ pode ser estimada consistentemente por $\hat{A}_{n}^{-1} \hat{B}_{n} \hat{A}_{n}^{-1}$, em que $\hat{A}_{n}$ é o Hessiano avaliado em $\hat{\theta}$

$$
\hat{A}_{n}=\frac{1}{n} \sum_{t=1}^{n}\left(\nabla F\left(x_{t} ; \hat{\theta}\right) \nabla F\left(x_{t} ; \hat{\theta}\right)^{\cdot}-\nabla^{2} F\left(x_{t} ; \hat{\theta}\right) \hat{\varepsilon}_{t}\right),
$$

com $\nabla F\left(x_{t} ; \hat{\theta}\right)=\partial F\left(x_{t} ; \hat{\theta}\right) / \partial \theta$, e $\hat{B}_{n}$ é o produto externo do gradiente

$$
\hat{B}_{n}=\frac{1}{n} \sum_{i=1}^{n} \hat{\varepsilon}_{l}^{2} \nabla F\left(x_{i} ; \hat{\theta}\right) \nabla F\left(x_{i} ; \hat{\theta}\right) .
$$

A estimação pode ser feita usando qualquer procedimento de otimização nãolinear (ver Quandt (1983), Hamilton (1994), Hendry (1995)). A escolha dos valores iniciais do algoritmo de otimização merece uma atenção particular.

Para valores fixos de $\gamma$ e $c$, o modelo STAR fica linear nos parâmetros autoregressivos $\phi_{1}$ e $\phi_{2}$, parecido com o modelo SETAR. Dessa forma, dado $\gamma$ e $c$, os estimadores de mínimos quadrados ordinários de $\phi=\left(\phi_{1}^{\prime}, \phi_{2}^{\prime}\right)^{\prime}$ podem ser obtidos por

$$
\hat{\phi}(\gamma, c)=\left(\sum_{t=1}^{n} x_{t}(\gamma, c) x_{t}(\gamma, c)^{\prime}\right)^{-1}\left(\sum_{t=1}^{n} x_{t}(\gamma, c) y_{t}\right),
$$

em que $x_{t}(\gamma, c)=\left(x_{t}^{\prime}\left(1-G\left(y_{t-1} ; \gamma, c\right)\right), x_{t}^{\prime} G\left(y_{t-1} ; \gamma, c\right)\right)^{\prime}$ e a notação $\phi(\gamma, c)$ é usada para indicar que o estimador de $\phi$ é condicionado em relação a $\gamma$ e $c$. Os resíduos podem ser computados por $\hat{\varepsilon}_{t}=y_{t}-\hat{\phi}(\gamma, c)^{\prime} x_{t}(\gamma, c)$ com a variância associada $\hat{\sigma}^{2}(\gamma, c)=n^{-1} \sum_{t=1}^{n} \hat{\varepsilon}_{t}^{2}(\gamma, c)$. Um método conveniente de obter valores iniciais adequados para o algoritmo de otimização não-linear seria, portanto, um grid search sobre $\gamma$ e $c$, e selecionar os parâmetros que obtiverem a menor estimativa da variância dos resíduos $\hat{\sigma}^{2}(\gamma, c)$.

Uma maneira de simplificar o problema de estimação é se concentrar na função de soma de quadrados (ver Leyborne, Newbold \& Vougas (1998)). Como o 
modelo STAR é linear nos parâmetros autoregressivos, dados $\gamma$ e $c$, a função de soma de quadrados $Q_{n}(\theta)$ pode ser dada por

$$
Q_{n}(\gamma, c)=\sum_{t=1}^{n}\left(y_{t}-\phi(\gamma, c)^{\prime} x_{t}(\gamma, c)\right)^{2} .
$$

Em relação a estimação do parâmetro de suavidade $\gamma$, é difícil obter uma estimativa precisa desse parâmetro. Uma razão para isso é que para valores grandes de $\gamma$, a forma da função logística (4.4) varia pouco. Dessa forma, para obter uma estimativa acurada de $\gamma$ é preciso obter muitas observações na vizinhança próxima do limiar $c$. Como isso raramente ocorre, a estimativa de $\gamma$ em geral é imprecisa e aparece insignificante pela estatística $t$. Logo, a insignificância da estimativa de $\gamma$ não deve ser interpretada como uma evidência contra a presença de não-linearidade do tipo STAR. Isso deve ser verificado por outros diagnósticos, que serão discutidos adiante.

\subsection{Teste de Não-Linearidade de Mudança de Regime}

Quando consideramos modelos de mudança de regime, devemos questionar se o regime adicional ${ }^{5}$ (relativamente a um único modelo linear AR) acrescenta poder explicativo para o comportamento da série temporal. Uma possível maneira de avaliar isso seria tomar o modelo linear como a hipótese nula e o modelo de mudança de regime como a alternativa. No caso do modelo de dois regimes, as hipóteses nula e alternativa podem ser expressas como

$$
\begin{aligned}
& H_{0}: \phi_{1}=\phi_{2} \\
& H_{1}: \phi_{i, 1} \neq \phi_{i, 2} \text { para pelo menos um } i \in\{0, \ldots, p\}
\end{aligned}
$$

em que $\phi_{i}$ são os parâmetros autoregressivos.

\footnotetext{
${ }^{5}$ Ou os regimes adicionais, no caso de múltiplos regimes;
} 
Já no caso de modelos de três ou mais regimes, eles possuem o problema de parâmetros de perturbação não-identificados (ver Franses \& van Djik (2000)), que faz com que não seja possível obter uma distribuição assintótica das estatísticas de teste.

Para testar linearidade contra a hipótese alternativa de um modelo SETAR, podemos definir uma razão de verossimilhanças ou uma estatística $\mathrm{F}$ que testa as restrições dadas pela hipótese nula em (4.20), obtendo

$$
F(\hat{c})=n\left(\frac{\tilde{\sigma}^{2}-\hat{\sigma}^{2}}{\hat{\sigma}^{2}}\right),
$$

em que $\widetilde{\sigma}^{2}$ é uma estimativa da variância dos resíduos sob a hipótese nula de linearidade, $\widetilde{\sigma}^{2}=\sum_{t=1}^{n} \widetilde{\varepsilon}_{t}^{2} \operatorname{com} \widetilde{\varepsilon}_{t}=y_{t}-\hat{\phi}^{\prime} x_{t}$, e $\hat{\sigma}^{2}$ é a variância dos resíduos estimada por $\hat{\sigma}^{2}=\hat{\sigma}^{2}(\hat{c})$, dado em (4.12). Como $F(\hat{c})$ é uma transformação monotônica de $\hat{\sigma}^{2}$ e $\hat{c}$ minimiza a variância dos resíduos sobre o conjunto $C$ de possíveis limiares, $F(\hat{c})$ equivale ao supremo sobre esse conjunto $C$ da estatística teste $F(c)$,

$$
F(\hat{c})=\sup _{c \in C} F(c),
$$

em que

$$
F(c)=n\left(\frac{\tilde{\sigma}^{2}-\hat{\sigma}^{2}(c)}{\hat{\sigma}^{2}(c)}\right)
$$

A estatística $F(c)$ pode também ser computada como um $n R^{2}$ com $R^{2}$ sendo o coeficiente de determinação de uma regressão artificial de $\widetilde{\varepsilon}_{t}$ sobre $x_{t} I\left(y_{t-1} \leq c\right)$ e $x_{t} I\left(y_{t-1}>c\right)$. Portanto, $F(c)$ possui distribuição assintótica $\chi^{2} \operatorname{com} p+1$ graus de liberdade.

Para testar linearidade contra a hipótese alternativa de um modelo STAR, além da igualdade dos parâmetros AR nos dois regimes, $H_{0}: \phi_{1}=\phi_{2}$, a hipótese nula de linearidade pode também ser expressa como $H_{0}^{\prime}: \gamma=0$. Se $\gamma=0$, a função logística (4.4) é igual a 0,5 para todos os valores de $y_{t-1}$ e o modelo STAR se reduz 
a um modelo AR com parâmetros $\left(\phi_{1}+\phi_{2}\right) / 2$. Para qualquer uma das formulações da hipótese nula, o modelo contém parâmetros não-identificados. Por exemplo, para $H_{0}$ os parâmetros $\gamma$ e $c$ são os parâmetros de perturbação não-identificados.

Para resolver o problema da identificação, Franses \& van Djik (2000) sugerem a utilização de um Teste Multiplicador de Lagrange (LM) que possui uma distribuição assintótica $\chi^{2}$.

Considere o modelo STAR dado em (4.6) e re-escrito como

$$
y_{t}=\frac{1}{2}\left(\phi_{1}+\phi_{2}\right)^{\prime} x_{t}+\left(\phi_{2}-\phi_{1}\right)^{\prime} x_{t} G^{*}\left(y_{t-1} ; \gamma, c\right)+\varepsilon_{t},
$$

em que $G^{*}\left(y_{t-1} ; \gamma, c\right)=G\left(y_{t-1} ; \gamma, c\right)-1 / 2$. Note que sob a hipótese nula de $\gamma=0$, $G^{*}\left(y_{t-1} ; \gamma, c\right)=0$. Luukkonen, Saikkonen \& Teräsvirta (1988) sugerem aproximar a função $G^{*}\left(y_{t-1} ; \gamma, c\right)$ por uma aproximação de polinômio de Taylor de primeira ordem ao redor de $\gamma=0$, isto é

$$
T_{1}\left(y_{t-1} ; \gamma, c\right) \approx G^{*}\left(y_{t-1} ; 0, c\right)+\left.\gamma \frac{\partial G^{*}\left(y_{t-1} ; \gamma, c\right)}{\partial \gamma}\right|_{\gamma=0}=\frac{1}{4} \gamma\left(y_{t-1}-c\right),
$$

Substituindo $G^{*}($.$) por T_{1}($.$) em (4.24) e arrumando os termos, obtemos o$ modelo da regressão auxiliar

$$
y_{t}=\beta_{0,0}+\beta_{0}^{\prime} \widetilde{x}_{t}+\beta_{1}^{\prime} \widetilde{x}_{t} y_{t-1}+\eta_{t},
$$

em que $\tilde{x}_{t}=\left(y_{t-1}, \ldots, y_{t-p}\right)^{\prime}$ e $\beta_{j}^{\prime}=\left(\beta_{1, j}, \ldots, \beta_{p, j}\right), j=0,1$. Franses \& van Djik (2000) mostram que as relações entre os parâmetros do modelo de regressão auxiliar (4.26) e os parâmetros do modelo STAR (4.24) podem ser dadas por

$$
\begin{aligned}
& \beta_{0,0}=\left(\phi_{0,1}+\phi_{0,2}\right) / 2-\frac{1}{4} \gamma c\left(\phi_{0,2}-\phi_{0,1}\right), \\
& \beta_{1,0}=\left(\phi_{1,1}+\phi_{1,2}\right) / 2-\frac{1}{4} \gamma\left(c\left(\phi_{1,2}-\phi_{1,1}\right)-\left(\phi_{0,2}-\phi_{0,1}\right)\right), \\
& \beta_{i, 0}=\left(\phi_{i, 1}+\phi_{i, 2}\right) / 2-\frac{1}{4} \gamma c\left(\phi_{i, 2}-\phi_{i, 1}\right), i=2, \ldots, p, \\
& \beta_{i, 1}=\frac{1}{4} \gamma c\left(\phi_{i, 2}-\phi_{i, 1}\right), i=1, \ldots, p .
\end{aligned}
$$


As equações acima mostram que a restrição $\gamma=0$ implica $\beta_{i, 1}=0$ para $i=1, \ldots$, p. Assim, testar a hipótese nula $H_{0}^{\prime}: \gamma=0$ em (4.24) equivale testar a hipótese nula $H_{0}^{\prime \prime}: \beta_{1}=0$ em (4.26). Assim, sob a hipótese nula de linearidade, a estatística de teste possui uma distribuição assintótica $\chi^{2} \operatorname{com} p$ graus de liberdade. Chamamos esse teste de teste do tipo LM (para mais detalhes, ver Franses \& van Djik (2000)).

Para amostras pequenas, Franses \& van Djik (2000) recomendam a utilização da versão F do teste LM. Teräsvirta (1994) sugere que o teste do tipo LM pode também ser usado para selecionar a variável de transição apropriada no modelo STAR. A estatística é computada para diversas variáveis de transição candidatas e aquela que possuir o menor $p$-valor será selecionada como a verdadeira variável de transição.

\subsection{Teste de Não-Linearidade Restante}

Nem todas as estatísticas de teste que foram desenvolvidas no contexto de modelos ARMA são aplicáveis para os resíduos de modelos não-lineares. Vamos discutir nesta seção alguns testes de diagnóstico que podem ser usados para avaliar modelos com mudança de regime.

Considere o modelo não-linear autoregressivo de ordem $p$,

$$
y_{t}=F\left(x_{i} ; \theta\right)+\varepsilon_{t},
$$

em que $x_{t}=\left(1, y_{t-1}, \ldots, y_{t-p}\right)^{\prime}$ e $F\left(x_{t} ; \theta\right)$ é uma função não-linear geral dos parâmetros $\theta$, que tem derivadas contínuas até $2^{\mathrm{a}}$ ordem. Um teste LM para a q-ésima ordem do coeficiente de autocorrelação de $\varepsilon$, pode ser obtido por $n R^{2}$, em que $R^{2}$ é o coeficiente de determinação da regressão entre $\hat{\varepsilon}_{t}$ e $\hat{z}_{t} \equiv \partial F\left(x_{t} ; \hat{\theta}\right) / \partial \theta$ e $q$ resíduos defasados $\hat{\varepsilon}_{t-1}, \ldots, \hat{\varepsilon}_{t-q}$, em que os circunflexos indicam que as quantidades são 
estimativas sob a hipótese nula de independência da seqüência $\varepsilon_{t}$. A estatística teste resultante tem distribuição assintótica $\chi^{2} \operatorname{com} q$ graus de liberdade.

No caso do modelo SETAR, ele não satisfaz a condição de que $F\left(x_{i} ; \theta\right)$ possui derivadas contínuas até $2^{\mathrm{a}}$ ordem. Uma maneira de contornar esse problema seria aproximar o SETAR por um STAR ao impor $\gamma$ igual a algum valor grande porém finito. Nesse caso, a derivada parcial com relação a $\gamma$ da função de regressão deve ser omitida da regressão auxiliar, uma vez que esse parâmetro está fixado.

Uma importante questão em modelos não-lineares de séries temporais é se o modelo proposto capta adequadamente todas as características de não-linearidade da série que está sendo analisada. Uma maneira de verificar isso é aplicar um teste de não-lineridade restante para um modelo estimado. Para os modelos SETAR e o STAR, uma abordagem natural seria especificar a hipótese alternativa da nãolinearidade restante como a presença de um regime adicional.

Segundo Franses \& van Djik (2000), há um teste LM de não-linearidade restante que pode ser usado apenas para o modelo STAR, sem a necessidade de estimar o modelo mais complicado. Para o modelo SETAR, o teste de nãolinearidade restante necessariamente envolve a estimação do modelo de múltiplos regimes.

Para o modelo SETAR, segundo Franses \& van Djik (2000), pode-se aplicar a metodologia usada para o teste de linearidade contra a alternativa de um SETAR para cada uma das subamostras definidas pelo limiar $\hat{c}$, usando a estatística de teste (4.22). Assim, quando a estatística de teste indicar a presença de um regime adicional, as estimativas do modelo de 3 regimes estão prontamente disponíveis ao combinarmos os resultados da estimação para o modelo SETAR de 2 regimes com aqueles para o modelo de 2 regimes na sub-amostra onde a linearidade é rejeitada. Segundo Franses \& van Djik (2000), enquanto a estimativa do segundo limiar $\hat{c}_{2}$ é consistente, a estimativa do primeiro limiar $\hat{c}_{1}$ não é. Para obter uma estimativa do primeiro limiar, necessita-se fazer um "procedimento de repartição" (repartitioning 
step), em que o modelo SETAR de 2 regimes é estimado na sub-amostra definida por $y_{t-1} \leq \hat{c}_{2}$ se $\hat{c}_{1}<\hat{c}_{2}$ e na sub-amostra definida por $y_{t-1}>\hat{c}_{2}$ se $\hat{c}_{1}>\hat{c}_{2}$ (para mais detalhes ver Bai \& Perron (1998)).

No caso do modelo STAR, Eitrheim \& Teräsvirta (1996) desenvolveram uma estatística LM para testar um modelo STAR de 2 regimes contra a alternativa de um modelo de 3 regimes que pode ser escrito como

$$
y_{t}=\phi_{1}^{\prime} x_{t}+\left(\phi_{2}-\phi_{1}\right)^{\prime} x_{t} G_{1}\left(y_{t-1} ; \gamma_{1}, c_{1}\right)+\left(\phi_{3}-\phi_{2}\right)^{\prime} x_{t} G_{2}\left(y_{t-1} ; \gamma_{2}, c_{2}\right)+\varepsilon_{t},
$$

em que $x_{t}=\left(1, \widetilde{x}_{t}\right)^{\prime}, \widetilde{x}_{t}=\left(y_{t-1}, \ldots, y_{t-p}\right)^{\prime}$, em que tanto $G_{1}$ como $G_{2}$ são dados por (4.4) e assumimos que $c_{1}<c_{2}$ sem perda de generalidade. A hipótese nula de um modelo de 2 regimes pode ser expressa por $H_{0}: \gamma_{2}=0$. Esse teste possui problemas de identificação parecidos com os problemas de testar a hipótese nula de linearidade contra a alternativa de um modelo STAR de 2 regimes discutido anteriormente. A solução é a mesma: a função de transição $G_{2}\left(y_{t-1} ; \gamma_{2}, c_{2}\right)$ é substituída por uma aproximação de polinômio de Taylor ao redor do ponto $\gamma_{2}=0$. No caso de uma aproximação de terceira ordem, o modelo auxiliar resultante é dado por

$$
y_{t}=\beta_{0}^{\prime} x_{t}+\left(\phi_{2}-\phi_{1}\right) x_{t} G_{1}\left(y_{t-1} ; \gamma_{1}, c_{1}\right)+\beta_{1}^{\prime} \tilde{x}_{t} y_{t-1}+\beta_{2}^{\prime} \tilde{x}_{t} y_{t-1}^{2}+\beta_{3}^{\prime} \tilde{x}_{t} y_{t-1}^{3}+\eta_{t}
$$

em que os $\beta_{j}, j=0,1,2,3$, são funções dos parâmetros $\phi_{1}, \phi_{3}, \gamma_{2}$ e $c_{2}$. A hipótese nula $H_{0}: \gamma_{2}=0$ se torna $H_{0}^{\prime}: \beta_{1}=\beta_{2}=\beta_{3}=0$ em (4.33). A estatística do teste pode ser computada como $n R^{2}$ a partir da regressão auxiliar dos resíduos $\hat{\varepsilon}_{t}$, obtidos da estimação do modelo, sob a hipótese nula, sobre as derivadas parciais da função de regressão com relação aos parâmetros $\phi_{1}, \phi_{2}, \gamma_{1}$ e $c_{1}$ do modelo de 2 regimes, e em relação aos regressores $\tilde{x}_{t} y_{t-1}^{j}, j=1,2,3$. A estatística resultante tem distribuição assintótica $\chi^{2}$ com $3 p$ graus de liberdade (para mais detalhes, ver Eitrheim \& Teräsvirta (1996)). 


\section{Capítulo 5}

\section{Extensão dos modelos de memória longa: o FI-STAR e o FI-BREAK}

\subsection{Modelo FI-STAR}

Algumas séries econômicas, tais como a taxa de desemprego americana, possuem tanto memória longa como não-linearidade. Uma tentativa de captar essas duas características ao mesmo tempo seria útil, uma vez que vários estudos revelam que se pode tentar aplicar um modelo de memória longa para dados nãolineares, e vice-versa. Por exemplo, Granger e Teräsvirta (1999) e Davidson (2000) demonstram que modelos não-lineares podem gerar séries temporais que podem ser ajustadas utilizando modelos de memória longa. Por outro lado, dados gerados a partir de modelos de memória longa podem parecer ter propriedades nãolineares (ver Andersson et al. (1999)).

Parece, portanto, interessante tentar capturar ambas as características de memória longa e não-linearidade em um único modelo de série temporal, para poder verificar a importância relativa de cada uma delas.

Considere o modelo que captura memória longa em uma série $y_{t}$

$$
(1-L)^{d} y_{t}=x_{t}
$$

e o modelo STAR, descrito no capítulo anterior, para $y_{t}$ dado por

$$
y_{t}=\left(\phi_{1,0}+\phi_{1,1} y_{t-1}+\ldots+\phi_{1, p} y_{t-p}\right)\left(1-G\left(s_{t} ; \gamma, c\right)\right)+\left(\phi_{2,0}+\phi_{2,1} y_{t-1}+\ldots+\phi_{2, p} y_{t-p}\right)\left(G\left(s_{t} ; \gamma, c\right)\right)+\varepsilon_{t}
$$


em que $\varepsilon_{t}$ é um processo ruído branco e a função de transição $G(s ; \gamma, c)$ usualmente é assumida como a função logística

$$
G\left(s_{t} ; \gamma, c\right)=\left(1+\exp \left\{-\gamma\left(s_{t}-c\right)\right\}\right)^{-1} \operatorname{com} \gamma>0,
$$

em que $s_{\imath}$ é a variável de transição.

Como foi visto no Capítulo 4, a variável de transição $s_{t}$ pode ser uma variável endógena defasada $\left(s_{t}=y_{t-l}\right.$ para algum inteiro $\left.l>0\right)$, uma variável exógena $\left(s_{t}=z_{t}\right)$ ou uma função (possivelmente não-linear) de variáveis endógenas defasadas, ou seja, $s_{t}=h\left(y_{t-1}, \ldots, y_{t-l} ; \alpha\right)$ para alguma função $h($.$) , que depende do$ vetor $(q \times 1)$ de parâmetros $\alpha$, e $l \geq 1$. A variável de transição também pode ser uma tendência linear $\left(s_{t}=t\right)$, que faz com que (5.2) passe a ser um modelo AR com mudança suave nos parâmetros.

Para tentar capturar características de não-linearidade e de memória longa simultaneamente, van Dijk et al. (2002) sugeriram o seguinte modelo:

$$
\begin{aligned}
& (1-L)^{d} y_{t}=x_{t} \\
& x_{t}=\phi_{1}^{\prime} w_{t}\left(1-G\left(s_{t} ; \gamma, c\right)\right)+\phi_{2}^{\prime} w_{t}\left(G\left(s_{t} ; \gamma, c\right)\right)+\varepsilon_{t}
\end{aligned}
$$

em que $x_{t}$ é um processo estacionário de segunda ordem, $w_{t}=\left(1, x_{t-1}, \ldots, x_{t-p}\right)$, $\phi_{i}=\left(\phi_{i, 0}, \phi_{i, 1}, \ldots, \phi_{i, p}\right)^{\prime}, i=1,2$, e $G\left(s_{t} ; \gamma, c\right)$ é a função de transição logística dada em (5.3). Assumimos que $\varepsilon_{t}$ é uma seqüência de diferença de martingais com relação ao passado da série até $t-1$, que é denotado por $\Omega_{t-1}=\left\{y_{t-1}, y_{t-2}, \ldots, y_{t-(p-1)}, y_{t-p}\right\}$, ou seja, $E\left[\varepsilon_{t} / \Omega_{t-1}\right]=0$. Por simplicidade, assumimos que $E\left[\varepsilon_{t}^{2} / \Omega_{t-1}\right]=\sigma^{2}$.

No modelo (5.4), as dinâmicas de curto-prazo, que são determinadas em grande parte pelos coeficientes $A R$, podem variar à medida que os coeficientes $A R$ podem oscilar entre $\phi_{1}$ e $\phi_{2}$, dependendo do valor da função de transição logística $G\left(s_{t} ; \gamma, c\right)$. Isso faz com que o modelo seja potencialmente útil para capturar as características de não-linearidade e de memória longa da série temporal $y_{t}$. 
O modelo FI-STAR ${ }^{6}$ (5.4) pode ser expresso como um modelo STAR de ordem infinita como

$$
y_{t}=\left(\phi_{1,0}+\sum_{j=1}^{\infty} \pi_{1, j} y_{t-j}\right)\left(1-G\left(s_{t} ; \gamma, c\right)\right)+\left(\phi_{2,0}+\sum_{j=1}^{\infty} \pi_{2, j} y_{t-j}\right) G\left(s_{t} ; \gamma, c\right)+\varepsilon_{t},
$$

em que $\pi_{i}(L)=\phi_{i}(L)(1-L)^{d}, i=1,2, \operatorname{com} \pi_{i}(L)=1-\pi_{i, 1} L-\pi_{i, 2} L^{2}-\ldots$, e $\phi_{i}(L)$ é definida analogamente.

Nas Figuras 5.1 e 5.2 abaixo temos duas simulações de 200 observações de um modelo FI-STAR de dois regimes dado por (5.1) e (5.2), com parâmetros $\phi_{0,1}=4$, $\phi_{1,1}=0.8$ para o primeiro regime e $\phi_{0,2}=2$ e $\phi_{1,2}=0.05$ para o segundo regime; utilizamos $\gamma=1, \gamma=25, \varepsilon_{t} \sim N(0,1), s_{t}=y_{t-1}, c=0, d=0,1$ e $d=0,4$.

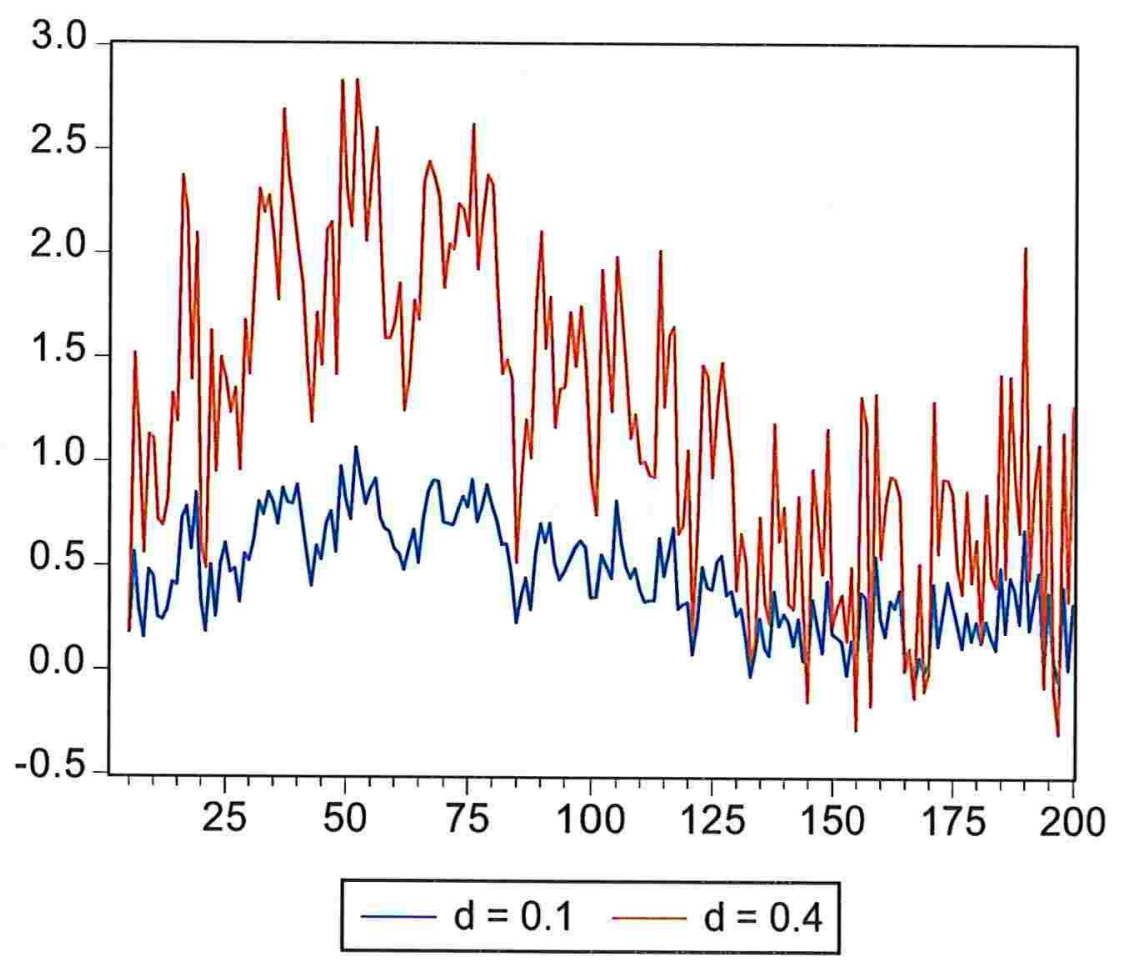

Figura 5.1 - Simulação de um FI-STAR de dois regimes dado por (5.1) e (5.2), com parâmetros $\phi_{0,1}=4, \phi_{1,1}=0.8, \phi_{0,2}=2, \phi_{1,2}=0.05$ e $\gamma=1$

\footnotetext{
${ }^{6}$ Fractionally Integrated - Smooth Treshold Auto-Regressive, para mais detalhes ver van Dijk et al.(2002).
} 


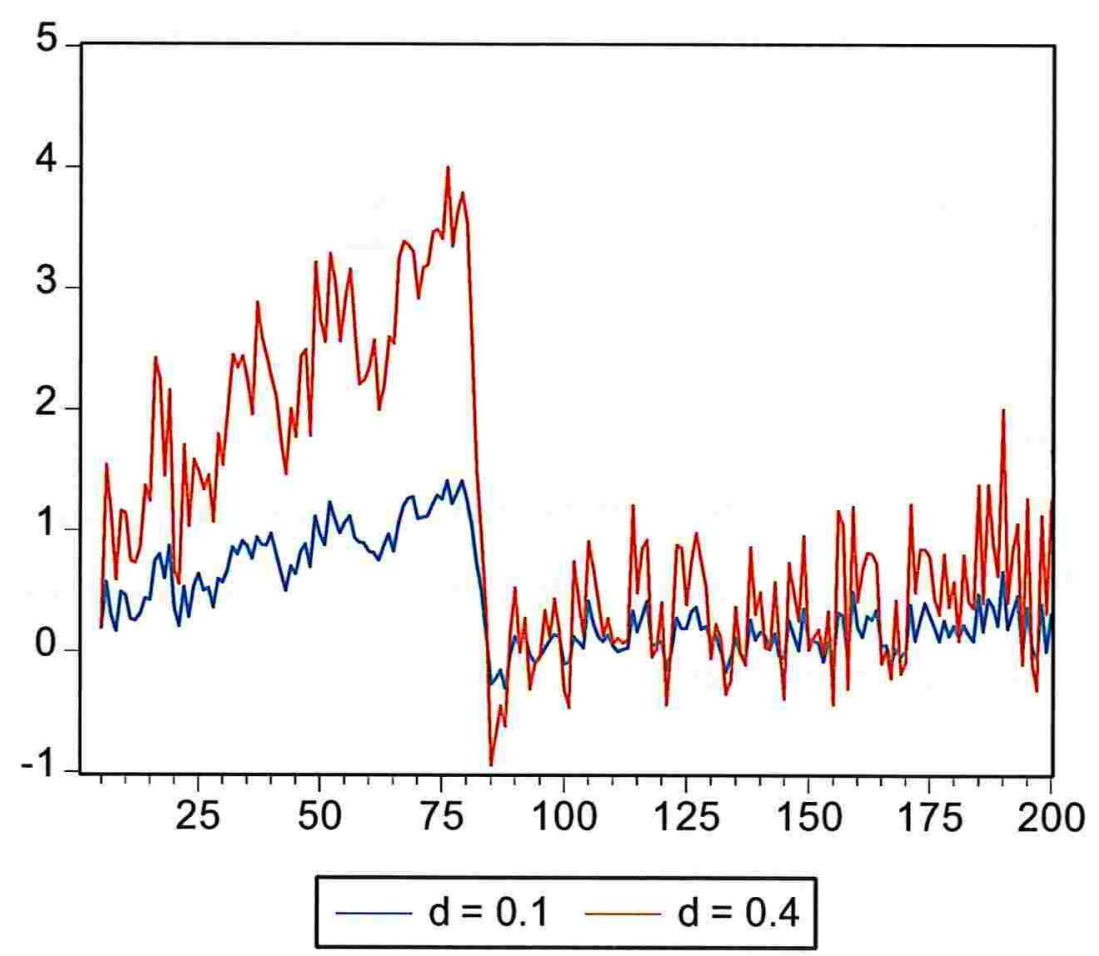

Figura 5.2 - Simulação de um FI-STAR de dois regimes dado por (5.1) e (5.2), com parâmetros $\phi_{0,1}=4, \phi_{1,1}=0.8, \phi_{0,2}=2, \phi_{1,2}=0.05$ e $\gamma=25$

A especificação do modelo FI-STAR é feita usando a estratégia do "específico para o geral" para construir modelos não-lineares de séries temporais (seguindo a recomendação de Granger (1993)). Isso implica começar com um modelo simples ou restrito e ir prosseguindo para modelos mais complicados, apenas se os testes de má-especificação indicarem que o modelo é inadequado.

O procedimento é feito da seguinte maneira:

(i) Especifica-se um modelo linear ARFI apropriado com ordem autoregressiva $p(\operatorname{ARFI}(\mathrm{p}))$ para a série temporal em questão;

(ii) Testa-se a hipótese nula de linearidade contra a alternativa de um modelo FI-STAR. Se a hipótese de linearidade for rejeitada, selecionase a variável de transição apropriada, $s_{t}$ (por exemplo, $y_{t-1}$ ). 
(iii) Estimam-se os parâmetros no modelo FI-STAR.

(iv) Avalia-se o modelo usando testes de especificação e outras medidas de diagnóstico.

Vamos discutir com mais detalhes os passos (ii) e (iii).

\subsubsection{Teste de Linearidade}

Considere novamente o modelo descrito em (5.4), onde agora a função de transição $G(s, ; \gamma, c)$ é dada pela função logística geral de $k$-ésima ordem

$$
G\left(s_{i} ; \gamma, c\right)=\left(1+\exp \left\{\gamma \prod_{i=1}^{k}\left(s_{t}-c_{i}\right)\right\}\right)^{-1} \operatorname{com} \gamma>0, c_{1} \leq \ldots \leq c_{k},
$$

sendo $k \geq 1$ o número de regimes. As restrições adicionais nos parâmetros $c_{1} \leq \ldots \leq c_{k}$ são restrições de identificação. Para $k$ ímpar, o valor da função de transição logística (5.6) está limitado entre 0 e $1, \operatorname{com} G\left(s_{t} ; \gamma, c\right) \rightarrow 0$ à medida que $s_{t} \rightarrow-\infty$ e $G\left(s_{t} ; \gamma, c\right) \rightarrow 1$ à medida que $s_{\imath} \rightarrow+\infty$. Por outro lado, para $k$ par, há $k$ transições suaves de $G\left(s_{t} ; \gamma, c\right)$ entre o mínimo e o máximo local à medida que $s_{t}$ cresce de $-\infty$ para $+\infty$. Dessa forma, o uso dessa função de transição é vantajoso, pois ela permite capturar diversas formas de mudança de regime ao variarmos o valor de $k$ (ver Teräsvirta (1998)).

Vamos apresentar um teste de linearidade contra a alternativa de um modelo FI-STAR. A hipótese nula de linearidade pode ser expressa como a igualdade dos parâmetros autoregressivos nos dois regimes do modelo FI-STAR em (5.4), isto é, $H_{0}: \phi_{1, j}=\phi_{2, j}$ para todo $j=0,1, \ldots, p$. Como vimos no Capítulo 4 , o problema desse teste é a presença de parâmetros de perturbação não-identificados sob a hipótese nula, à medida que os parâmetros da função de transição $(\gamma$ e $c)$ saem do modelo quando a hipótese nula é válida. Note que a hipótese nula pode ser escrita 
alternativamente como $H_{0}^{\prime}: \gamma=0$, que também dá origem a um modelo ARFI linear, com parâmetros iguais a uma média dos parâmetros dos dois regimes.

Como vimos no Capítulo 4, para superar o problema de identificação, usa-se a abordagem de Luukkonen et al. (1988) e aproxima-se $G\left(s_{t} ; \gamma, c\right)$ em (5.6) por uma expansão de Taylor de primeira ordem ao redor da hipótese nula $H_{0}^{\prime}: \gamma=0$,

$$
\begin{aligned}
T_{1}\left(s_{t} ; \gamma, c\right) & =G\left(s_{t} ; 0, c\right)+\left.\gamma \frac{\partial G\left(s_{t} ; \gamma, c\right)}{\partial \gamma}\right|_{\gamma=0}+R_{1}\left(s_{t} ; \gamma, c\right) \\
& =\frac{1}{2}+\frac{\gamma}{4}\left(s_{t}-c\right)+R_{1}\left(s_{t} ; \gamma, c\right),
\end{aligned}
$$

em que $R_{1}\left(s_{t} ; \gamma, c\right)$ é um termo de resto. Substituindo $G($.$) por T_{1}($.$) em (5.4) e re-$ arranjando os termos, obtemos a regressão auxiliar:

$$
x_{t}=\beta_{0}^{\prime} w_{t}+\beta_{1}^{\prime} w_{t} s_{t}+\ldots+\beta_{k}^{\prime} w_{t} s_{t}^{k}+e_{t}
$$

em que $w_{t}=\left(1, x_{t-1}, \ldots, x_{t-p}\right)$ e $e_{t}=\varepsilon_{t}+\left(\phi_{2}-\phi_{1}\right)^{\prime} w_{t} R_{1}\left(s_{t} ; \gamma, c\right)$.

Note que sob a hipótese nula, $R_{\mathrm{l}}\left(s_{t} ; \gamma, c\right)=0$ e $e_{t}=\varepsilon_{t}$. Conseqüentemente, o termo de resto da aproximação de Taylor não afeta as propriedades dos resíduos sob a hipótese nula e, portanto, também não afeta a distribuição teórica para as estatísticas do teste de linearidade a ser apresentado a seguir.

As relações entre os parâmetros $\left(\beta_{i, 0}, \beta_{i, 1}, \ldots, \beta_{i, p}\right)^{\prime}, i=0,1, \ldots, k$, na regressão auxiliar (5.8), e os parâmetros no modelo original FI-STAR são tais que a hipótese nula pode ser reformulada como $H_{0}^{\prime \prime}: \beta_{i}=0, \mathrm{i}=1, \ldots, \mathrm{k}$.

A hipótese nula de linearidade em (5.8) pode ser convenientemente testada por meio de um Teste de Multiplicador de Lagrange (LM). Assumindo que os erros sejam normalmente distribuídos com variância $\sigma^{2}$, a log-verossimilhança condicional para a observação t é dada por 


$$
l_{t}=-\frac{1}{2} \ln 2 \pi-\frac{1}{2} \ln \sigma^{2}-\frac{e_{t}^{2}}{2 \sigma^{2}} .
$$

Como a matriz de informação é diagonal, a variância do erro $\sigma^{2}$ pode ser assumida como fixa. As derivadas parciais restantes, avaliadas sob a hipótese nula, são dadas por

$$
\begin{aligned}
& \left.\frac{\partial l_{t}}{\partial \beta_{i}}\right|_{H_{0}^{\prime \prime}}=\frac{1}{\sigma^{2}} \hat{\varepsilon}_{t} w_{t} s_{t}^{i}, \quad i=0,1, \ldots, k, \\
& \left.\frac{\partial l_{t}}{\partial d}\right|_{H_{0}^{\prime \prime}}=\left.\frac{1}{\sigma^{2}} e_{t} \frac{\partial e_{t}}{\partial d}\right|_{H_{0}^{\prime \prime}}=-\frac{1}{\sigma^{2}} \hat{\varepsilon}_{t} \sum_{j=1}^{t-1} \frac{\hat{\varepsilon}_{t-j}}{j},
\end{aligned}
$$

em que $\hat{\varepsilon}_{t}$ são os resíduos obtidos sob a hipótese nula (modelo ARFI).

A estatística do teste de Multiplicador de Lagrange para testar

$$
\begin{aligned}
& H_{0}^{\prime \prime}: \beta_{i}=\underset{\sim}{0}, \mathrm{i}=1, \ldots, \mathrm{k} \\
& H_{1}^{\prime \prime}: \text { Pelo menos um } \beta_{i} \neq \underset{\sim}{0}, \mathrm{i}=1, \ldots, \mathrm{k}
\end{aligned}
$$

é dada por

$$
L M=\hat{l}_{\beta}^{\prime} \operatorname{cov}\left(\hat{l}_{\beta}\right)^{-1} \hat{l}_{\beta}
$$

em que $l_{\beta}=\left(l_{\beta, 1}, \ldots, l_{\beta, n}\right)^{\prime}$, com $l_{\beta, t}=\left(\frac{\partial l_{t}}{\partial \beta_{1}}, \ldots, \frac{\partial l_{t}}{\partial \beta_{k}}\right)^{\prime}$ e $n$ denotando o tamanho da amostra. Os circunflexos indicam que todos os elementos de $l_{\beta}$ são avaliados sob a hipótese nula.

As expressões para as derivadas parciais da log-verossimilhança dada em (5.10) e (5.11) sugerem que a estatística teste pode ser computada da seguinte forma:

(i) Estima-se um modelo ARFI, obtêm-se os resíduos $\hat{\varepsilon}_{\text {, }}$ e computa-se a soma dos quadrados dos resíduos sob a hipótese nula, isto é, $S Q R_{0}=\sum_{t=1}^{n} \hat{\varepsilon}_{t}^{2}$. 
(ii) Faz-se uma regressão dos resíduos $\hat{\varepsilon}_{t}$ em $w_{t,}-\sum_{j=1}^{t-1} \frac{\hat{\varepsilon}_{t-j}}{j}$, e nos regressores auxiliares $w_{t} s_{t}^{i}, i=1, \ldots, k$ e computa-se a soma dos quadrados dos resíduos sob a hipótese alternativa, $S Q R_{1}$.

(iii) Computa-se a versão $\chi^{2}$ da estatística do teste como

$$
L M_{k}=\frac{\left(S Q R_{0}-S Q R_{1}\right)}{\left(S Q R_{0} / n\right)}
$$

ou a versão $F$ como

$$
L M_{k}=\frac{\left(S Q R_{0}-S Q R_{1}\right) /(k(p+1))}{S Q R_{1} /(n-(k+1)(p+1))} .
$$

Sob a hipótese nula, as versões $\chi^{2}$ e $F$ de $L M_{k}$ têm distribuições $\chi^{2}$ com $k(p+1)$ e $F$ com $k(p+1)$ e $n-(k+1)(p+1)$ graus de liberdade, respectivamente. Como usualmente, a versão $F$ da estatística teste é preferível à versão $\chi^{2} \mathrm{em}$ amostras pequenas, porque suas propriedades de tamanho e poder são melhores.

Note que a única diferença entre a estatística derivada acima e a do teste de um modelo linear AR contra a hipótese alternativa de um modelo STAR, visto no Capítulo 4, é a inclusão, na regressão auxiliar no passo 2 , do termo $-\sum_{j=1}^{t-1} \frac{\hat{\varepsilon}_{t-j}}{j}$, que surge a partir do gradiente da verossimilhança com respeito ao parâmetro de diferença fracionária $d$.

Finalmente, os testes LM derivados anteriormente são baseados em linearizações do modelo FI-STAR. Portanto, alguma informação sobre a forma da estrutura não-linear sob a hipótese alternativa é perdida, o que pode afetar adversamente o poder dos testes. Como uma alternativa, podem-se considerar testes (de razão de verossimilhanças ou Wald) de Andrews (1993) e Andrews e Ploberger (1994), usando a técnica de simulação desenvolvida por Hansen (1996) para obter valores críticos. 
Entretanto, a abordagem de Hansen (1996) pode ser computacionalmente complicada para testes do tipo não lineares STAR, se o modelo sob a hipótese alternativa contiver ao menos dois parâmetros de perturbação não-identificados.

Skalin (1998) investiga um teste utilizando um bootstrap paramétrico, mas conclui que ele possui propriedades de tamanho e poder piores do que os testes LM tradicionais. Portanto, adotamos os testes LM apresentado em (5.13) e (5.14).

\subsubsection{Estimação}

Para estimar os parâmetros nos modelos FI-STAR, van Dijk et al. (2002) modificaram o estimador de Beran (1995) de máxima verossimilhança aproximada (AML) apresentado no Capítulo 2, para permitir dinâmicas de mudança de regime. O estimador resultante equivale a minimizar a soma dos quadrados dos resíduos

$$
Q_{n}(\theta)=\sum_{t=1}^{n} e_{t}^{2}(\theta)
$$

em que $\theta=\left(\phi_{1}^{\prime}, \phi_{2}^{\prime}, d, \gamma, c\right)$ e os resíduos $e_{t}(\theta)$ são computados como

$$
e_{t}(\theta)=y_{t}-\left(\phi_{1,0}+\sum_{j=1}^{t+p-1} \pi_{1, j} y_{t-j}\right)\left(1-G\left(s_{t} ; \gamma, c\right)\right)-\left(\phi_{2,0}+\sum_{j=1}^{t+p-1} \pi_{2, j} y_{t-j}\right) G\left(s_{t} ; \gamma, c\right),
$$

em que $\pi_{1, j}$ e $\pi_{2, j}$, para $\pi_{i}(L)=\phi_{i}(L)(1-L)^{d}$, são os coeficientes autoregressivos na representação de ordem infinita STAR do modelo FI-STAR dada em (5.5).

Como vimos no Capítulo 2, para o modelo ARFI linear, o estimador AML é assintoticamente eficiente se os erros $\varepsilon_{t}$ forem normalmente distribuídos e, sob algumas condições de regularidade restritivas, ele é $\sqrt{n}$-consistente e assintoticamente normal. Não podemos assegurar eficiência do estimador AML para o modelo FI-STAR, mas consistência e normalidade assintótica seguem do 
fato de que o modelo FI-STAR satisfaz as condições M1-M7 de Wooldridge (1994, pp. 2653-2655).

Vamos verificar agora a escolha dos valores iniciais para o algoritmo de otimização e a estimação do parâmetro de suavidade $\gamma$. Note que quando o parâmetro de diferença fracionária $d$ é conhecido e os outros parâmetros da função de transição $G\left(s_{i} ; \gamma, c\right)$ são conhecidos, o modelo FI-STAR é linear nos parâmetros autoregressivos restantes. Dessa forma, condicionado a $d, \gamma$ e $c$, as estimativas de $\phi_{1}$ e $\phi_{2}$ podem ser obtidas por mínimos quadrados ordinários (MQO):

$$
\hat{\phi}(d, \gamma, c)^{\prime}=\left(\sum_{t=1}^{n} w_{t}(d, \gamma, c) w_{t}(d, \gamma, c)^{\prime}\right)^{-1}\left(\sum_{t=1}^{n} w_{t}(d, \gamma, c) x_{t}\right),
$$

onde $w_{t}=\left(1, x_{t-1}, \ldots, x_{t-p}\right), x_{t}=(1-L)^{d} y_{t}, w_{t}(d, \gamma, c)=\left(w_{t}^{\prime}\left(1-G\left(s_{t} ; \gamma, c\right)\right), w_{t}^{\prime} G\left(s_{t} ; \gamma, c\right)\right)^{\prime}$, e a notação $\hat{\phi}(d, \gamma, c)$ é usada para indicar que a estimativa de $\phi$ é condicional aos valores de $d, \gamma$ e $c$. Isso implica que a função da soma dos quadrados pode ser concentrada com relação a $\phi_{1}$ e $\phi_{2}$, como

$$
Q_{n}(d, \gamma, c)=\sum_{t=1}^{n}\left(x_{t}-\hat{\phi}(d, \gamma, c)^{\prime} w_{t}(d, \gamma, c)\right)^{2} .
$$

Isso reduz consideravelmente a dimensão do problema de estimação nãolinear, à medida que $Q_{n}(d, \gamma, c)$ precisa ser minimizada apenas com relação aos três parâmetros $d, \gamma$ e $c$. Além disso, a expressão acima sugere imediatamente que um grid search sobre $d$, $\gamma$ e $c$ é um método conveniente para obter valores iniciais para a otimização não-linear.

As observações feitas no Capítulo 4 para estimar o parâmetro de suavidade $\gamma$ do modelo STAR valem para o modelo FI-STAR. Como vimos anteriormente, pode parecer difícil estimar precisamente o parâmetro de suavidade entre regimes 
diferentes quando $\gamma$ é grande. Isso porque para grandes valores de $\gamma$, a função logística de transição $G\left(s_{t} ; \gamma, c\right)$ dada em (5.3) se aproxima de uma função indicadora. Para obter uma estimativa acurada de $\gamma$ é necessário ter muitas observações na vizinhança imediata do limiar $c$, uma vez que grandes mudanças em $\gamma$ têm apenas um pequeno efeito sobre a forma da função de transição.

A estimativa de $\gamma$ pode, portanto, ser muito imprecisa e freqüentemente se apresentar não-significativa quando julgada pela estatística $t$. Entretanto, isso não deve ser interpretado como uma evidência contra não-linearidade. Na verdade, a razão de a estatística $t$ não possuir a distribuição assintótica especificada sob a hipótese $\gamma=0$, deve-se aos problemas de identificação mencionados anteriormente.

\subsubsection{Testes de Má Especificação}

Os testes de autocorrelação nos resíduos, de não-linearidade restante e de constância nos parâmetros vistos no Capítulo 4 para o modelo STAR podem ser modificados para obtermos testes similares de má especificação para o modelo FISTAR, como foi sugerido por van Djik et al. (2002).

No caso do teste de autocorrelação nos resíduos, a hipótese nula de nãoautocorrelação em $\varepsilon_{1}$ de (5.4) pode ser testada contra a alternativa de dependência serial até a ordem $q$, dado por

$$
\varepsilon_{t}=\alpha_{1} \varepsilon_{t-1}+\ldots+\alpha_{q} \varepsilon_{t-q}+e_{t},
$$

com $e_{t} \sim$ i.i.d. $\left(0, \sigma^{2}\right)$. A hipótese nula é dada por $H_{0}: \alpha_{1}=\ldots=\alpha_{q}=0$ que, segundo Eitrheim \& Teräsvirta (1996), pode ser testada por um teste LM.

No caso do teste de não-linearidade restante, a hipótese nula de nãolinearidade restante pode ser testada contra a de um modelo FI-STAR de 3 regimes, que é obtida combinando (5.4) com 


$$
x_{t}=\phi_{1}^{\prime} w_{t}+\left(\phi_{2}-\phi_{1}\right)^{\prime} w_{t} G_{1}\left(s_{t}\right)+\left(\phi_{3}-\phi_{2}\right)^{\prime} w_{t} G_{2}\left(s_{t}\right)+e_{t},
$$

em que $G_{i}\left(s_{t}\right) \equiv G_{i}\left(s_{t} ; \gamma_{i}, c_{i}\right), i=1,2$ são funções logísticas como em (5.6). A hipótese nula de que não há não-linearidade restante é dada por $H_{0}: \phi_{2}=\phi_{3}$ ou por $H_{0}: \gamma_{2}=0$. Evidentemente, esse teste tem um problema de identificação similar ao encontrado quando testamos linearidade contra o modelo FI-STAR. Como foi sugerido por van Djik et al. (2002), a solução adotada é a mesma, ou seja, evitamos o problema de identificação ao substituir a segunda função de transição $G_{2}\left(s_{t}\right)$ por uma aproximação do polinômio de Taylor ao redor da hipótese nula, de forma que a hipótese nula possa ser testada por um teste LM padrão.

Se acreditarmos que a não-linearidade adicional possa ser determinada por uma variável diferente de $s_{1}$, segundo van Djik et al. (2002), um modelo para considerar a hipótese alternativa é o FI-STAR de 4 regimes, dado por (5.4) com

$$
x_{t}=\left[\dot{\phi}_{1}^{\prime} w_{t}\left(1-G_{1}\left(s_{1 t}\right)\right)+\dot{\phi}_{2} w_{t} G_{1}\left(s_{1 t}\right)\right]\left[1-G_{2}\left(s_{2 t}\right)\right]+\left[\left(1-G_{1}\left(s_{1 t}\right)\right) \dot{\phi}_{3} w_{t}+G_{1}\left(s_{1 t}\right) \dot{\phi}_{4}^{\prime} w_{t}\right] G_{2}\left(s_{2 \prime}\right)+e_{t},
$$

em que $s_{1, t} \equiv s_{t}$ (ver van Djik \& Franses (1999)). Um teste de não-linearidade restante, novamente, é obtido ao aproximarmos $G_{2}\left(s_{t}\right)$ por uma expansão do polinômio de Taylor ao redor da hipótese nula $H_{0}^{\prime}: \gamma_{2}=0$.

Um teste de constância nos parâmetros do modelo FI-STAR contra alternativa de mudança gradual nos parâmetros pode, também, ser baseado em (5.19) ao tomarmos $s_{2 t}=t$. Isso pode ser visto mais facilmente a partir da representação alternativa

$$
x_{t}=\phi_{1 t}^{\prime} w_{t}\left(1-G_{1}\left(s_{1 t} ; \gamma_{1}, c_{1}\right)\right)+\dot{\phi}_{2 t}^{\prime} w_{t} G_{1}\left(s_{1 t} ; \gamma_{1}, c_{1}\right)+e_{t}
$$

em que

$$
\begin{aligned}
& \phi_{1 t}=\left(1-G_{2}(t)\right) \phi_{1}+G_{2}(t) \phi_{3}, \mathrm{e} \\
& \phi_{2 t}=\left(1-G_{2}(t)\right) \phi_{2}+G_{2}(t) \phi_{4},
\end{aligned}
$$

Para os três testes de má especificação, a única diferença com os testes correspondentes para o modelo STAR (vistos no Capítulo 4) é que temos que 
incluir o gradiente de $e_{t}$ com relação ao parâmetro de diferença fracionária $d$, avaliado sob a hipótese nula, nas regressões auxiliares (vistas no Capítulo 4) que são usadas para computar as estatísticas de teste. Sob a hipótese nula $e_{t}=\varepsilon_{1}$, tal que

$$
\left.\frac{\partial e_{t}}{\partial d}\right|_{H_{0}}=\frac{\partial \varepsilon_{t}}{\partial d}=-\sum_{j}^{t-1} \frac{\varepsilon_{t-j}}{j} .
$$

Note que esse termo é idêntico à diferença entre os testes de linearidade contra os modelos STAR e FI-STAR alternativos, como foi discutido anteriormente.

\subsection{Modelo FI-BREAK}

Diversas séries temporais, inclusive séries financeiras, podem ser caracterizadas ou por memória longa ou por mudanças de nível. Por exemplo, taxas de inflação ou algumas séries de volatilidade financeira (ver Bos et al. (1999)). Alguns estudos como o de Granger \& Hyung (2001) e Diebold \& Inoue (2001) mostraram que uma aparente memória longa pode ser negligenciada por mudanças estruturais em uma série temporal, enquanto outros estudos indicam exatamente o efeito reverso.

Assim, resultados empíricos para algumas séries financeiras não devem ser vistos como surpresa caso modelos de memória longa e de quebra estrutural capturarem as mesmas características empíricas.

Dessa forma, há uma motivação para juntar os dois modelos em um único, uma vez que ambos os modelos individuais podem capturar um componente de memória longa até um certo ponto e, com isso, um modelo conjunto seria capaz de capturar todos componentes de memória longa.

Vamos explorar a possibilidade de que o modelo de quebra estrutural (ou modelo BREAK) e o processo $\operatorname{ARFIMA}(\mathrm{p}, \mathrm{d}, \mathrm{q})$ de memória longa possam ser resumidos em um único modelo, o modelo FI-BREAK. 
Como vimos no capítulo 3, um modelo relacionado de quebra estrutural muito usado é o STOPBREAK de Engle \& Smith (1999), dado por

$$
\begin{aligned}
& y_{t}=m_{t}+\varepsilon_{t} \\
& m_{t}=m_{t-1}+q_{t-1} \varepsilon_{t-1}
\end{aligned}
$$

em que a função $q_{t}$ é especificada como

$$
q_{t}=\frac{\left(\varepsilon_{t}+\ldots+\varepsilon_{t-s+1}\right)^{2}}{\gamma+\left(\varepsilon_{t}+\ldots+\varepsilon_{t-s+1}\right)^{2}}
$$

para algum $s>0$ e $\gamma>0$. Como vimos acima, este modelo inclui uma função de transição endógena para indicar quebras estruturais.

Dessa forma, para construir um modelo conjunto ARFIMA e de quebra estrutural, para uma série de dados temporais $y_{t}$, bastaria acrescentar uma diferença fracionária sobre $\left\{y_{t}\right\}$, obtendo

$$
\begin{aligned}
& (1-L)^{d} y_{t}=m_{t}+\varepsilon_{t} \\
& m_{t}=m_{t-1}+q_{t} \eta_{t}
\end{aligned}
$$

em que $q_{t}$ segue uma distribuição i.i.d. Bernoulli $(p)$. Por simplicidade, assumimos $\varepsilon_{t} \sim$ i.i.d. $\left(0, \sigma_{\varepsilon}{ }^{2}\right)$ e $\eta_{t} \sim$ i.i.d. $\left(0, \sigma_{\eta}{ }^{2}\right)$.

Generalizando, Hyung \& Franses (2005) utilizam a seguinte representação de um modelo FI-BREAK

$$
\begin{aligned}
& \alpha(L)(1-L)^{d} y_{t}=m_{t}+\varepsilon_{t} \\
& m_{t}=m_{t-1}+q_{t-1} \varepsilon_{t-1} \\
& q_{t}=\frac{\left(\varepsilon_{t}+\ldots+\varepsilon_{t-s+1}\right)^{2}}{\gamma+\left(\varepsilon_{t}+\ldots+\varepsilon_{t-s+1}\right)^{2}}
\end{aligned}
$$


em que $\alpha(L)=\left(1-\alpha_{1} L-\ldots-\alpha_{p} L^{p}\right)$.

Assumimos que $m_{0}$ é fixo e conhecido. Como vimos no Capítulo 4, esse termo determina a média não-condicional do processo.

A Figura 5.3 abaixo mostra duas simulações de 1000 observações do processo FI-BREAK descrito por

$$
\begin{aligned}
& (1-L)^{d} y_{t}=m_{t}+\varepsilon_{t} \\
& m_{t}=m_{t-1}+q_{t} \eta_{t}
\end{aligned}
$$

com $\varepsilon_{t} \sim N(0,1), \eta_{t} \sim N(0 ; 0,5), d=0,1$ e $d=0,4$, onde $q_{t}$ segue uma distribuição i.i.d. binomial, ou seja, $q_{t}=1$ com probabilidade $p=0,009$ e $q_{t}=0$ caso contrário. Como podemos notar na Figura 5.3 abaixo, quando $d=0,1$ a série possui um componente mais fraco de memória longa do que quando $d=0,4$.
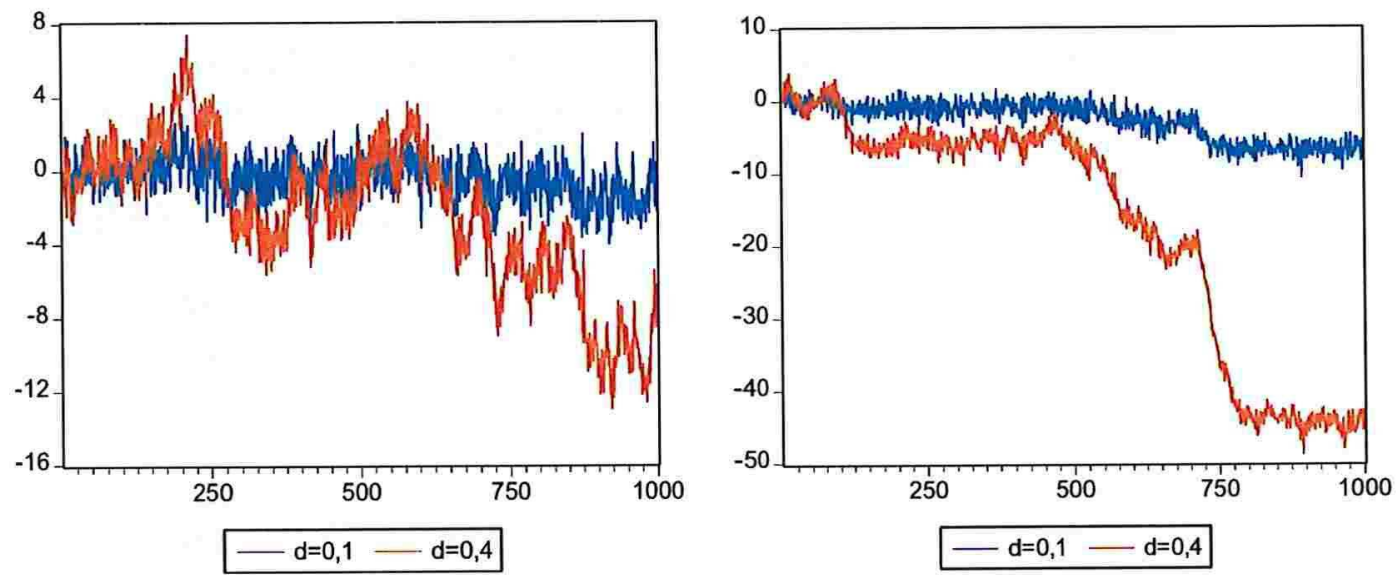

Figura 5.3 - Simulação de dois processos FI-BREAK

Note que o modelo (5.25) é uma generalização do FI-STAR de van Dijk et. al (2002), visto na seção anterior. Outra vantagem do FI-BREAK é que ele pode ser 
visto como uma junção de diversos modelos relacionados ao impormos certas restrições nos parâmetros:

(i) Quando $d=0$ e $\gamma \rightarrow \infty$, o modelo se transforma em um modelo AR(p). De fato, à medida que $\gamma \rightarrow \infty, q_{t}=0$ para todo $t$, o que implica $m_{t}=m_{0}$ para todo $t$. Além disso, se adicionalmente $d=0$, temos $\alpha(L) y_{t}=m_{0}+\varepsilon_{t}$.

(ii) Quando $0<d<1$ e $\gamma \rightarrow \infty$, o modelo torna-se um modelo $\operatorname{ARFI}(\mathrm{p}, \mathrm{d})$, que é $\alpha(L)(1-L)^{d} y_{t}=m_{0}+\varepsilon_{t}$.

(iii) Quando $d=0$ e $0<\gamma<\infty$, o modelo é o conhecido modelo STOPBREAK. Se $\gamma<\infty$, este processo contém uma quebra endógena suave, como foi visto no capítulo 3.

(iv) Quando $0<d^{-}<1$ e $0<\gamma<\infty$, o modelo FI-BREAK combina um modelo ARFI de memória longa e um modelo de quebra estrutural.

Os resultados estão resumidos na Tabela 5.1 .

Tabela 5.1 Modelos e espaço paramétrico - FI-BREAK

\begin{tabular}{|c|c|c|c|}
\hline & $d=0$ & $0<d<1$ & $\mathrm{~d}=1$ \\
\hline$\gamma \rightarrow 0$ & ARI(p, 1) & ARFI(p,1+d) & ARI $(p, 2)$ \\
\hline $0<\gamma<\infty$ & STOPBREAK & FI-BREAK & BREAK Integrado \\
\hline$\gamma \rightarrow \infty$ & $A R(p)$ & $A R F I(p, d)$ & ARI $(p, 1)$ \\
\hline
\end{tabular}

Por conveniência de estimação, foram consideradas apenas dinâmicas do tipo AR. Podemos re-escrever o modelo (5.25) na seguinte forma:

$$
\sum_{j=0}^{\infty} \pi_{j} \Delta y_{t-j}=\varepsilon_{t}-\theta_{t-1} \varepsilon_{t-1}
$$


em que $\theta_{t-1}=1-q_{t-1}$ e $\sum_{j=0}^{\infty} \pi_{j} L^{j}=\left(1-\alpha_{1} L-\ldots-\alpha_{p} L^{p}\right)(1-L)^{d}$. A partir do Teorema 3.1, pode-se mostrar que esse processo não-linear de médias móveis é invertível com probabilidade 1 se $P\left(q_{l}>0\right)>0$ e se

$$
q_{t}\left[1+\frac{\varepsilon_{t}}{q_{t}} \frac{\partial q_{t}}{\partial \varepsilon_{t}}\right]<2,
$$

com probabilidade 1 .

\subsubsection{Estimação}

Podemos estimar os parâmetros do modelo usando o método AML de Beran (1995) visto no Capítulo 2. De acordo com Hyung \& Franses (2005), o estimador AML para o modelo FI-BREAK é consistente e assintoticamente normal.

Para cada um dos possíveis modelos descritos pelo FI-BREAK, usamos os seguintes métodos de estimação:

(i) O modelo AR é estimado pelo método de mínimos quadrados, em que

$$
\alpha(L) y_{t}=\mu+\varepsilon_{t}
$$

onde $\alpha(L)=1-\alpha_{1} L-\ldots-\alpha_{p} L^{p}$.

(ii) O modelo ARFI é estimado pelo método de máxima verossimilhança aproximada, visto no capítulo 2. O modelo é dado por

$$
\alpha(L)(1-L)^{d} y_{t}=\mu+\varepsilon_{t}
$$

e centramos os dados antes de estimar $d$.

(iii) O modelo de quebra estrutural usa o método de Bai \& Perron (1998) e Bai \& Perron (2003), visto no capítulo 3, que consiste na estimação seqüencial dos pontos de quebra. Usamos esse modelo ao invés do modelo STOPBREAK, uma vez que, segundo Hyung \& Franses (2005), esse modelo de quebra é mais adequado em análises empíricas de dados de memória longa.

(iv) O modelo FI-BREAK de Hyung \& Franses (2005) usa o método de máxima verossimilhança aproximada (AML), visto no Capítulo 2. 


\section{Capítulo 6}

\section{Aplicação}

\subsection{Introdução}

Neste capítulo vamos ajustar os modelos FI-STAR e FI-BREAK a séries reais. Para a aplicação do FI-STAR, usaremos a série da taxa de desemprego norteamericana dessasonalizada, de janeiro de 1969 a junho de 2004 e a série de retornos absolutos diários do IBOVESPA, de 03.01.1995 a 22.11.2000; para o FI-BREAK, ajustaremos a série de retornos mensais da Treasure Bill americana, de janeiro de 1934 a dezembro de 2003 e a série do índice de preços do trigo de Beveridge, de 1500 a 1845.

\subsection{Aplicação do FI-STAR}

\subsubsection{Taxa de Desemprego Norte-Americana}

Considere a série mensal da taxa de desemprego norte-americana dessasonalizada, de janeiro de 1969 a junho de 2004, mostrada na Figura 6.1. 


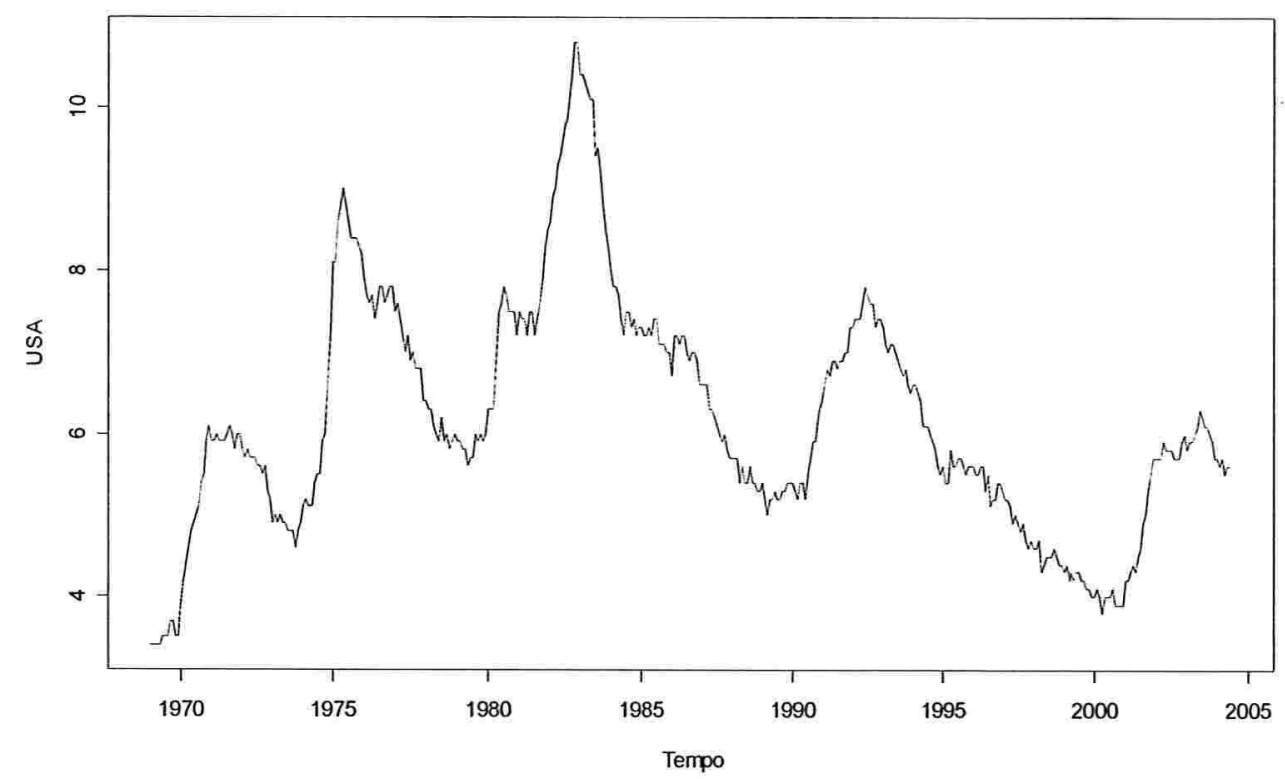

Figura 6.1 - Taxa de desemprego norte-americano dessasonalizada

Observando a função de autocorrelação na Figura 6.2, nota-se que ela decai a uma taxa hiperbólica, indicando a presença de memória longa. A estatística de teste R/S é igual a 2,9223, rejeitando a hipótese nula de que não há memória longa a um nível de 1\% de significância. O Quadro 6.1 mostra a saída do software SPLUS do teste R/S de memória longa, usando o comando "rosTest", do módulo FinMetrics (ver Anexo 1.1). 




Quadro 6.1 - Saída do teste R/S de memória longa usando o software S-PLUS.

Fazendo o teste GPH, também rejeitamos a hipótese nula de que não há memória longa a um nível de $1 \%$ de significância, com uma estatística de teste igual a 4,6812 e um $\hat{d}=0,8484$. O Quadro 6.2 mostra a saída do software S-PLUS do teste GPH de memória longa, usando o comando "gphTest", do módulo FinMetrics (ver Anexo 1.1).

\begin{tabular}{|c|}
\hline Test for Long Memory: GPH Test \\
Null Hypothesis: $\mathrm{d}=0$ \\
Test Statistics: \\
d 0.8484 \\
stat $4.6812^{* *}$ \\
* $:$ significant at $5 \%$ level \\
$* *$ significant at $1 \%$ level \\
Total Observ.: 426 \\
Number of Freq: 20 \\
\hline
\end{tabular}

Quadro 6.2 - Saída do teste GPH de memória longa usando o software S-PLUS. 


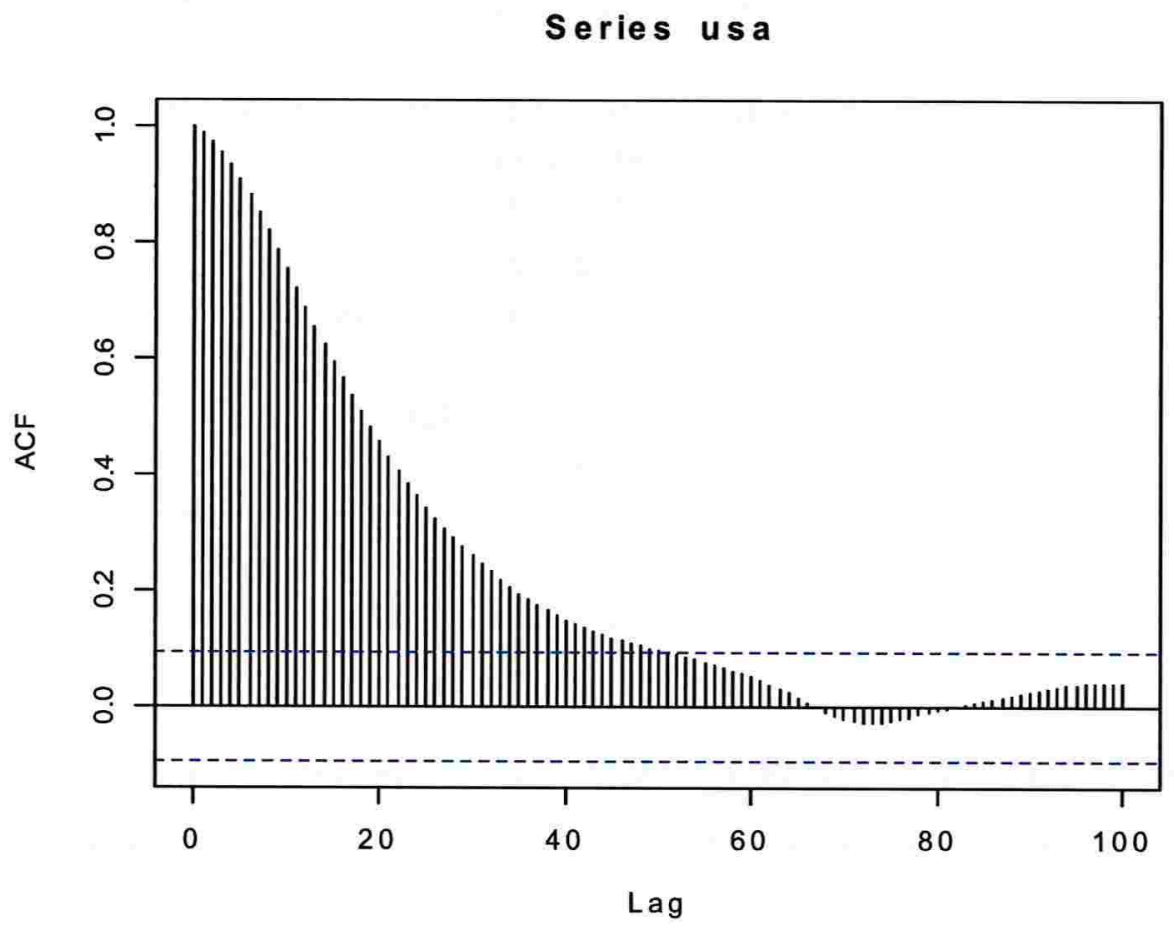

Figura 6.2 - Função de auto-correlação da taxa de desemprego norte-americana.

Como podemos notar no Quadro 6.2, $0.5<\hat{d}<1$, sugerindo que a taxa de desemprego norte-americana é não-estacionária, mas possui reversão à média.

Seguindo a especificação do FI-STAR delineada no Capítulo 5, começamos especificando um modelo ARFI para a série. Permitimos até a máxima ordem autoregressiva $\mathrm{p}=8$. O critério de BIC indica que um modelo ARFI com $\mathrm{p}=2$ é adequado para essa série. O Quadro 6.3 mostra uma saída do software S-PLUS, de uma estimação de máxima verossimilhança de um modelo $\operatorname{ARFI}(2, d)$, usando o comando "FARIMA", do módulo FinMetrics (ver Anexo 1.2). 


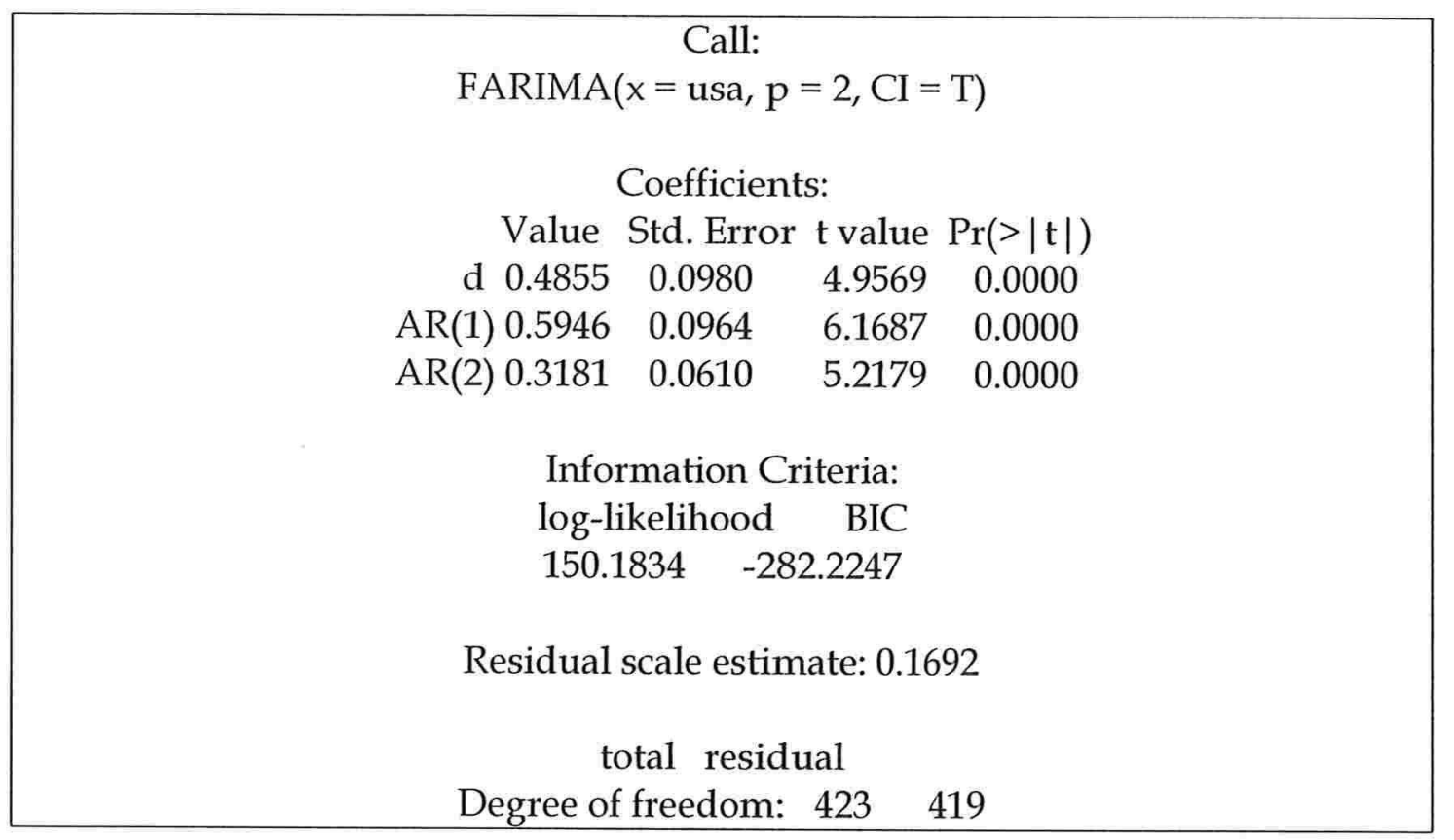

Quadro 6.3 - Saída de um ARFI(2,d) usando o software S-PLUS.

O Quadro 6.4 mostra a saída do software $R$, de uma estimação de máxima verossimilhança de um modelo $\operatorname{ARFI}(2, \mathrm{~d})$, usando o comando "fracdiff", da library "fracdiff" (ver Anexo 1.2). 


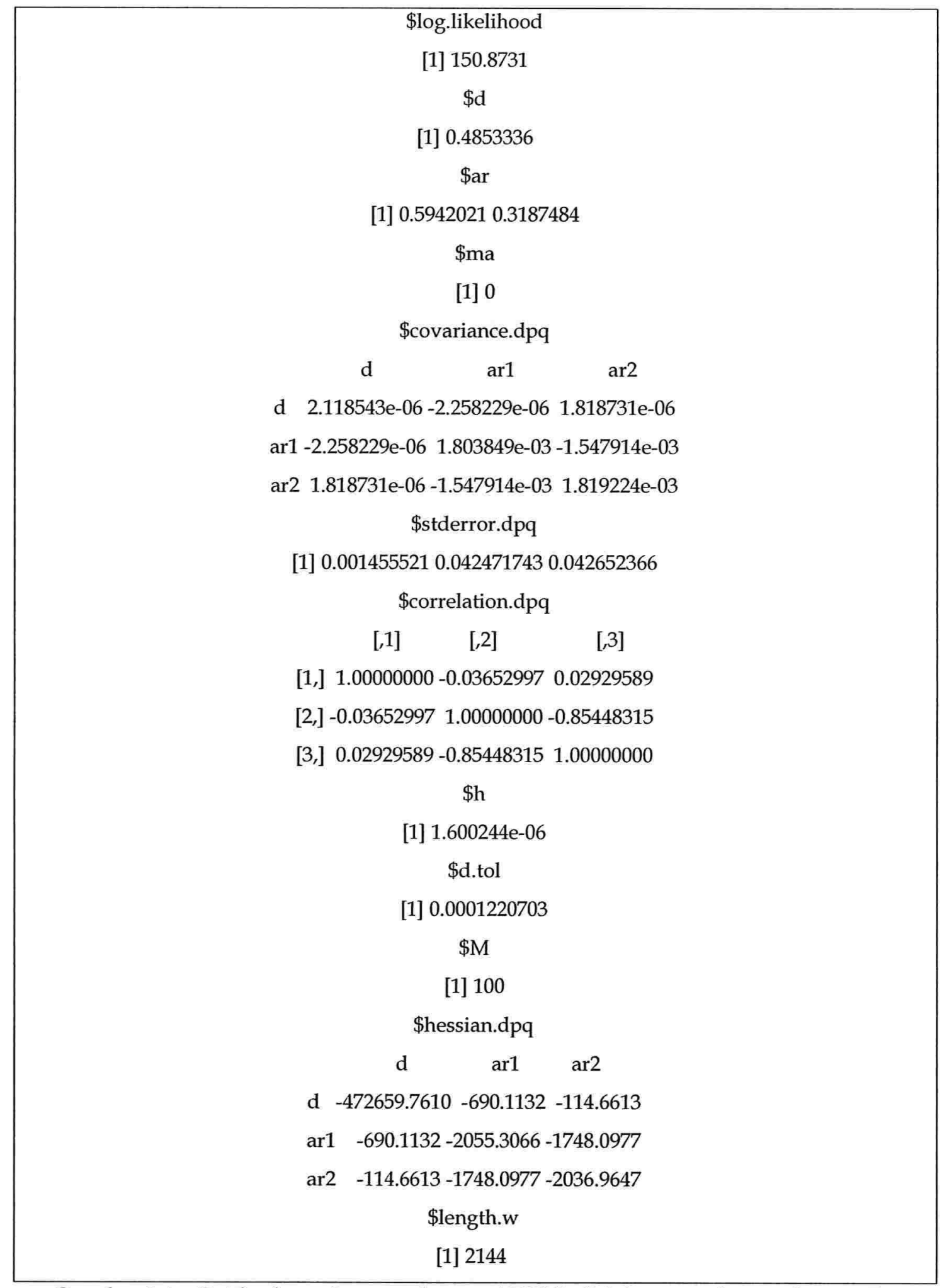

Quadro 6.4 - Saída da estimação de um $\operatorname{ARFI}(2, d)$ feita usando o software R. 
Obtemos o valor $\hat{d}$ igual a 0,4853336 usando o software $\mathrm{R}$, no caso do software S-PLUS, estimou-se um d igual a 0,4855. Fizemos o teste LM de nãolinearidade nos resíduos do modelo $\operatorname{ARFI}(2, \mathrm{~d})$. Como podemos notar na Tabela 6.1, rejeitamos a hipótese nula de linearidade a um nível de 5\% de significância, e $y_{t-2}$ é a variável de transição escolhida, pois possui um menor p-valor e, dessa forma, uma maior estatística de teste LM.

\begin{tabular}{|c|c|c|}
\hline Variável de Transição & $\begin{array}{c}\text { P-valores da Estatística de } \\
\text { LM F }\end{array}$ & $\begin{array}{c}\text { P-valores da Estatística de } \\
\text { LM } \chi^{2}\end{array}$ \\
\hline$y_{t}$ & 0,00 & $3,3 \mathrm{E}-12$ \\
\hline$y_{t-1}$ & 0,00 & $4,1 \mathrm{E}-12$ \\
\hline$y_{t-2}$ & 0,00 & $2,7 \mathrm{E}-12$ \\
\hline$y_{t-3}$ & 0,00 & $2,1 \mathrm{E}-11$ \\
\hline$y_{t-4}$ & 0,00 & $3,9 \mathrm{E}-11$ \\
\hline$y_{t-5}$ & 0,00 & $6,3 \mathrm{E}-11$ \\
\hline$y_{t-6}$ & 0,00 & $7,6 \mathrm{E}-11$ \\
\hline
\end{tabular}

Tabela 6.1 - Testes LM de linearidade.

Para fazer a estimação do FI-STAR, adotamos o algoritmo de estimação de van Djik (2002), descrito no Capítulo 5. Vamos usar o valor $\hat{d}$ estimado para diferenciar fracionariamente a série da taxa de desemprego norte-americana e estimamos um STAR para a série diferenciada. Assim, condicionado a d, $\gamma$ e c, o modelo FI-STAR se torna linear nos demais parâmetros. Dessa forma, pode-se estimar d, $\gamma$ e c por mínimos quadrados não-lineares e os demais coeficientes auto-regressivos dos dois regimes podem ser estimados por mínimos quadrados ordinários.

O Quadro 6.5 mostra uma saída do software $R$, de uma estimação de máxima verossimilhança de um STAR da série diferenciada fracionariamente, usando a library "tsDyn" e o comando "1star" (ver Anexo 1.3). 


\begin{tabular}{|c|}
\hline Non linear autoregressive model \\
\hline LSTAR model \\
\hline Coefficients: \\
\hline Low regime: \\
\hline phi1.0 phi1.1 phi1.2 \\
\hline-0.097373560 .294029930 .29186117 \\
\hline High regime: \\
\hline phi2.0 phi2.1 phi2.2 \\
\hline 0.039300590 .668660450 .20114188 \\
\hline Smoothing parameter: gamma $=16.75$ \\
\hline Threshold \\
\hline Variable: $Z(t)=+(0) X(t)+(0) X(t-1)+(1) X(t-2)$ \\
\hline Value: -0.07185 \\
\hline Residuals: \\
\hline Min 1Q Median 3Q Max \\
\hline$-0.661399-0.099059-0.0109330 .0986410 .661611$ \\
\hline Fit: \\
\hline residuals variance $=0.02651, \mathrm{AIC}=-1530, \mathrm{MAPE}=334 \%$ \\
\hline Coefficient(s): \\
\hline Estimate Std. Error $t$ value $\operatorname{Pr}(>|t|)$ \\
\hline phi1.0 $-0.097374 \quad 0.029894-3.25730 .001125$ ** \\
\hline phi1.1 $0.294030 \quad 0.0920393 .19460 .001400^{* *}$ \\
\hline phi1.2 $0.291861 \quad 0.0743573 .92528 .667 \mathrm{e}-05^{* * *}$ \\
\hline phi2.0 $\quad 0.039301 \quad 0.019280 \quad 2.03840 .041512$ * \\
\hline phi2.1 $0.668660 \quad 0.06453810 .3607<2.2 \mathrm{e}-16^{* * *}$ \\
\hline phi2.2 $\quad 0.201142 \quad 0.0674402 .98250 .002859^{* *}$ \\
\hline gamma 16.7545528 .2363002 .03420 .041928 * \\
\hline $\begin{array}{llll}-0.071847 & 0.051711 & -1.3894 & 0.164714\end{array}$ \\
\hline Signif. codes: $0^{1 * * * 1} 0.001^{1 * * 1} 0.01^{* * 1} 0.05$ '.' $0.1^{\prime \prime}{ }^{\prime} 1$ \\
\hline Non-linearity test of full-order LSTAR model against full-order AR model \\
\hline $\mathrm{F}=0.80977 ; \mathrm{p}$-value $=0.48897$ \\
\hline Threshold \\
\hline Variable: $Z(t)=+(0) X(t)+(0) X(t-1)+(1) X(t-2)$ \\
\hline
\end{tabular}

Quadro 6.5 - Saída da estimação do modelo STAR usando o software R. 
A Tabela 6.2 mostra os coeficientes estimados pelo FI-STAR. Na Figura 6.3, comparamos o FI-STAR estimado com a série original diferenciada fracionariamente. Nota-se que o modelo FI-STAR ajusta-se bem a essa série. $\mathrm{O}$ parâmetro de suavidade $\hat{\gamma}$ é muito grande, indicando que o modelo FI-STAR se aproxima de um FI-TAR, com uma mudança abrupta de regime. Fizemos o teste de não-linearidade restante, cujos resultados são mostrados na Tabela 6.3, e não rejeitamos a hipótese nula de que o modelo está corretamente especificado.

\begin{tabular}{ccccc}
\hline Coeficientes & Estimativa & Erro Padrão & Estatística $t$ & P-Valor \\
\hline$\phi_{0,1}$ & $-0,097$ & 0,030 & $-3,257$ & 0,001 \\
$\phi_{1,1}$ & 0,294 & 0,092 & 3,195 & 0,001 \\
$\phi_{2,1}$ & 0,292 & 0,074 & 3,925 & 0,087 \\
$\phi_{0,2}$ & 0,039 & 0,019 & 2,038 & 0,042 \\
$\phi_{1,2}$ & 0,669 & 0,065 & 10,361 & $<2,2 \mathrm{e}-16$ \\
$\phi_{2,2}$ & 0,201 & 0,067 & 2,983 & 0,003 \\
$\hat{\gamma}$ & 16,755 & 8,236 & 2,034 & 0,042 \\
$\hat{c}$ & $-0,072$ & 0,052 & $-13,894$ & 0,165 \\
$\hat{d}$ & 0,486 & 0,098 & 49,569 & 0,000 \\
\hline
\end{tabular}

Tabela 6.2 - Coeficientes estimados pelo FI-STAR.

\begin{tabular}{|c|c|}
\hline Variável de Transição & P-valores da Estatística de LM $\chi^{2}$ \\
\hline$y_{t}$ & 0,96 \\
\hline$y_{t-1}$ & 0,98 \\
\hline$y_{t-2}$ & 0,96 \\
\hline$y_{t-3}$ & 0,97 \\
\hline$y_{t-4}$ & 0,91 \\
\hline$y_{t-5}$ & 0,86 \\
\hline$y_{t-6}$ & 0,96 \\
\hline
\end{tabular}

Tabela 6.3 - Teste LM $\chi^{2}$ de não-linearidade restante. 
De acordo com a Tabela 6.2, o modelo ajustado, com os erros padrão em parênteses, é dado por:

$$
\begin{aligned}
& (1-L)^{\hat{d}} y_{t}=x_{t}, \quad \hat{\mathrm{d}}=0,486 \\
& (0,098) \\
& x_{t}=\left(-0,097+0,294 x_{t-1}+0,292 x_{t-2}\right) \times\left(1-G\left(y_{t-2} ; \hat{\gamma} ; \hat{c}\right)\right)+\left(0,039+0,669 x_{t-1}+0,201 x_{t-2}\right) \times G\left(y_{t-2} ; \hat{\gamma} ; \hat{c}\right), \\
& (0,030)(0,092) \quad(0,074) \quad(0,019)(0,065) \quad(0,067) \\
& G\left(y_{t-2} ; \hat{\gamma} ; \hat{c}\right)=\left(1+\exp \left\{-16,755\left(y_{t-2}-(-0,072)\right)\right\}\right)^{-1}, \\
& (8,236) \quad(0,052)
\end{aligned}
$$

A Figura 6.3 apresenta as séries real e ajustada pelo modelo FI-STAR.

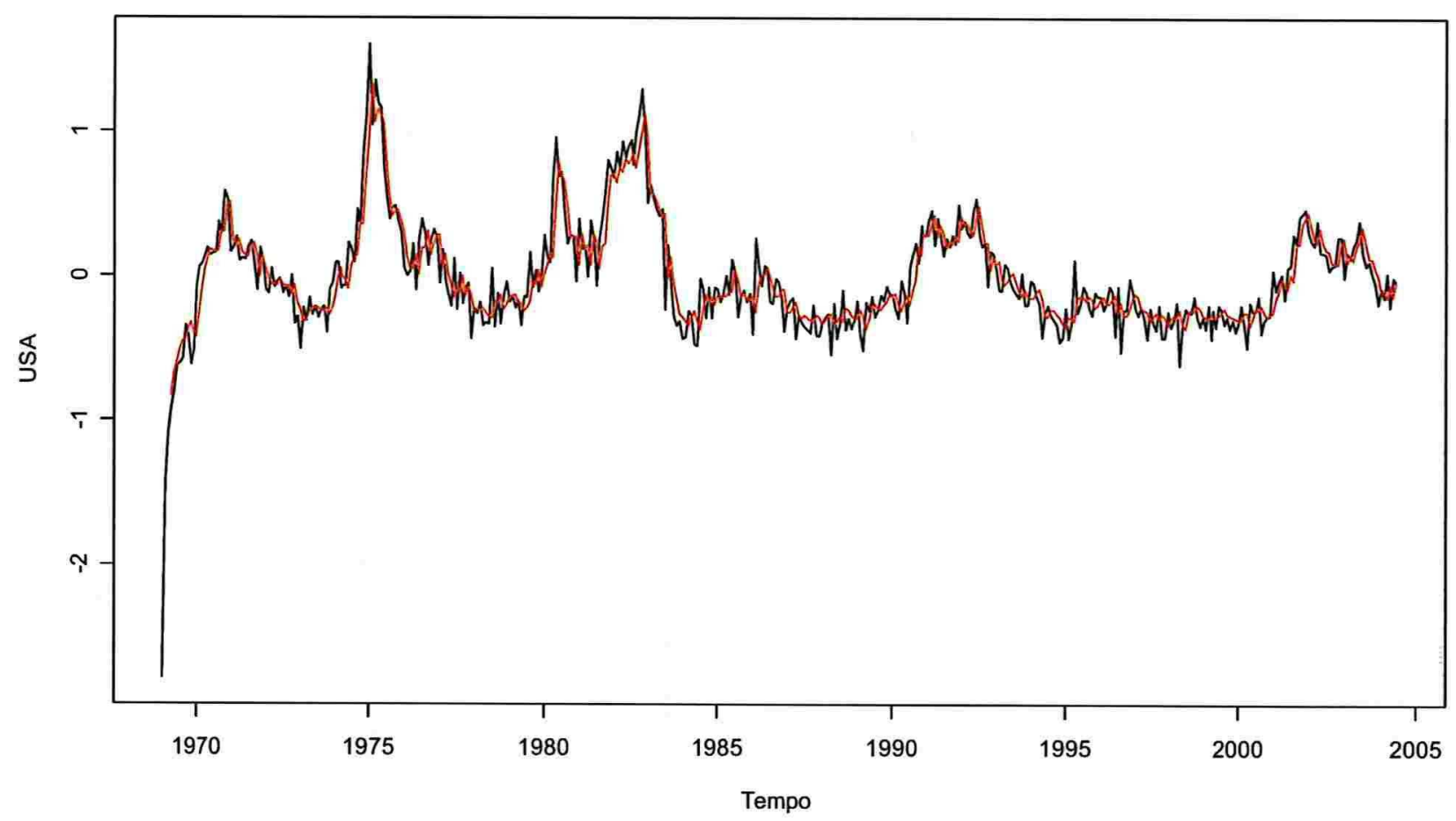

Figura 6.3 - Taxa de desemprego americana diferenciada fracionariamente (--) e modelo FI-STAR ajustado (--). 
A Figura 6.4 mostra a série original da taxa de desemprego americana com a projeção não-atualizada fora da amostra, em vermelho, do FI-STAR.

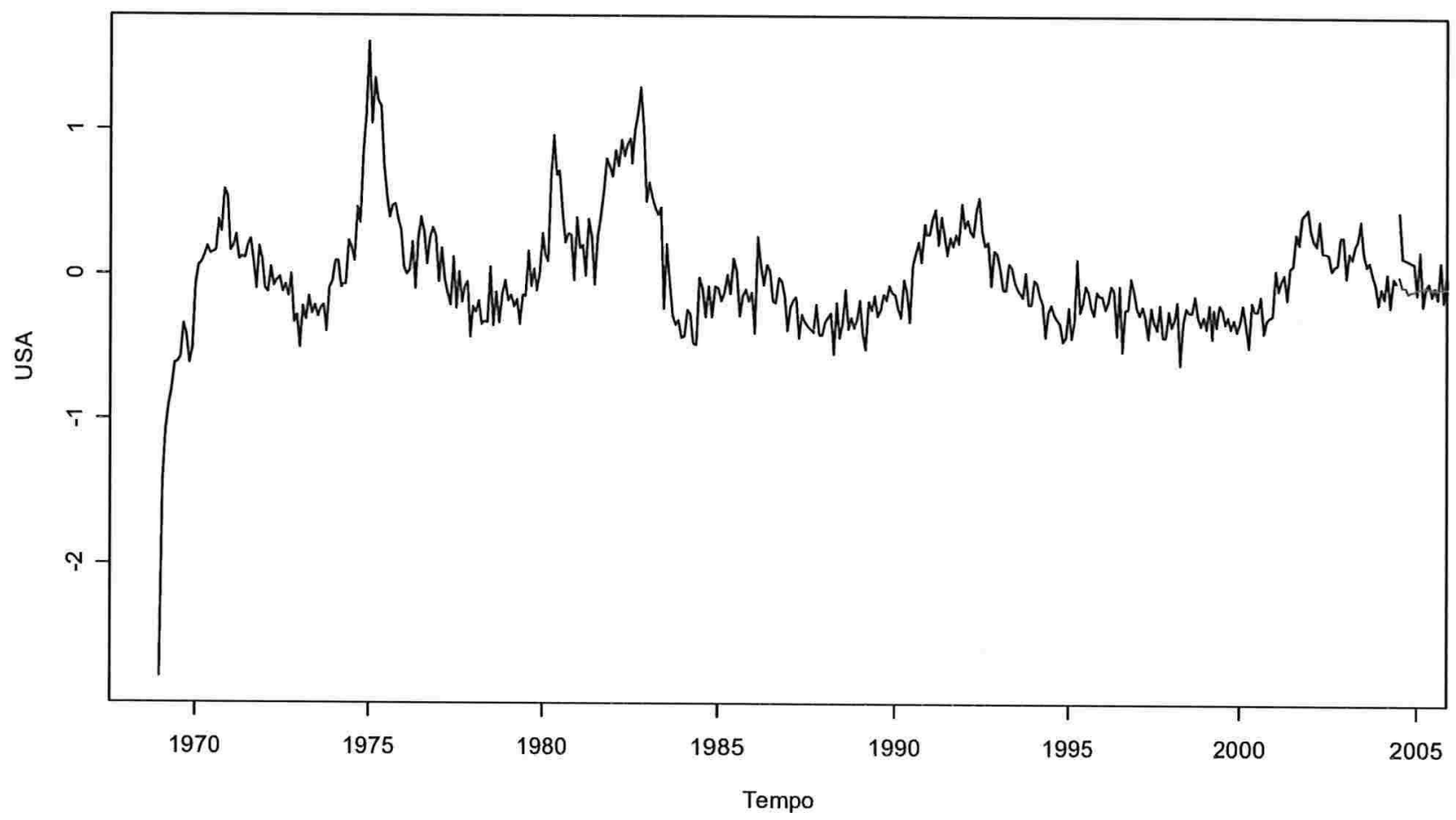

Figura 6.4 - Taxa de desemprego americana (--) e projeção do FI-STAR (--).

Para efeito de comparação, também ajustamos os modelos:

(i) FI-TAR:

$$
\begin{aligned}
& (1-L)^{\hat{d}} y_{t}=x_{t}, \quad \hat{\mathrm{d}}=0,486 \\
& (0,098) \\
& x_{t}=\left(-0,062+0,374 x_{t-1}+0,296 x_{t-2}\right) \times\left(1-I\left(y_{t-2}>0,004\right)\right)+ \\
& (0,016)(0,067) \quad(0,063) \\
& +\left(0,038+0,670 x_{t-1}+0,202 x_{t-2}\right) \times I\left(y_{t-2}>0,004\right), \\
& (0,017)(0,065) \quad(0,066) \\
& \hat{\sigma}^{2}=0,0265 .
\end{aligned}
$$


(ii) STAR para $d=0$ :

$$
\begin{aligned}
& y_{t}=\left(-0,120+1,352 y_{t-1}+0,033 y_{t-2}-0,352 y_{t-3}\right) \times\left(1-G\left(y_{t-12} ; \hat{\gamma} ; \hat{c}\right)\right)+\left(0,045+1,028 y_{t-1}+0,156 y_{t-2}-0,193 y_{t-3}\right) \times G\left(y_{t-12} ; i\right. \\
& \begin{array}{llllll}
(0,126)(0,116) & (0,187) & (0,126) & (0,047)(0,050) & (0,074) & (0,050)
\end{array} \\
& G\left(y_{t-11} ; \hat{\gamma} ; \hat{c}\right)=\left(1+\exp \left\{108595\left(y_{t-11}-4,966\right)\right\}\right)^{-1}, \\
& (222,75) \quad(0,107)
\end{aligned}
$$

$\hat{\sigma}^{2}=0,0271$.

(iii) STAR para $d=1$ :

$(1-L) y_{t}=z_{t}$,

$z_{t}=\left(0,013-0,077 z_{t-1}+0,128 z_{t-2}+0,233 z_{t-3}+0,297 z_{t-4}\right) \times\left(1-G\left(y_{t-3} ; \hat{\gamma} ; \hat{c}\right)\right)+\left(-0,114+0,936 z_{t-1}+0,464 z_{t-2}\right) \times G\left(y_{t-3} ; \hat{\gamma}\right.$ $\begin{array}{llllll}(0,013)(0,070) & (0,057) & (0,054) & (0,087) & (0,136)(0,360) & (0,231)\end{array}$

$G\left(y_{t-3} ; \hat{\gamma} ; \hat{c}\right)=\left(1+\exp \left\{-10,463\left(y_{t-3}-0,327\right)\right\}\right)^{-1}$,

$(4,643) \quad(0,094)$

$\hat{\sigma}^{2}=0,0263$.

(iv) $\operatorname{ARI}(2,1)$ :

$(1-L) y_{t}=z_{t}$,

$x_{t}=0,005+0,117 x_{t-1}+0,234 x_{t-2}$,

$(0,013)(0,047) \quad(0,047)$

$\hat{\sigma}^{2}=0,0297$.

A Tabela 6.4 relaciona o AIC e a variância dos resíduos para cada um dos modelos ajustados. Para calcular e comparar o AIC desses modelos, controlamos a amostra, e usamos a amostra do modelo com o maior número de parâmetros. 


\begin{tabular}{ccc}
\hline Modelo & AIC & $\hat{\sigma}^{2}$ \\
\hline FI-STAR & -1.514 & 0,0265 \\
FI-TAR & -1.516 & 0,0265 \\
STAR, $d=0$ & -1.503 & 0,0271 \\
STAR, $d=1$ & -1.515 & 0,0263 \\
ARI $(2,1)$ & -1.478 & 0,0297 \\
\hline
\end{tabular}

Tabela 6.4 - Coeficientes estimados pelo FI-STAR

Como podemos notar na Tabela 6.4, o STAR com $d=1$, o FI-STAR e o FI-TAR possuem um ajuste melhor para essa série. E, como observamos anteriormente, o fato de $\hat{\gamma}$ ser muito grande justifica que o ajuste do modelo FI-STAR se aproxime de um FI-TAR.

\subsubsection{Retornos Absolutos do IBOVESPA}

Considere a série diária dos retornos absolutos do IBOVESPA, de 03.01.1995 a 22.11.2000, apresentada na Figura 6.5. 


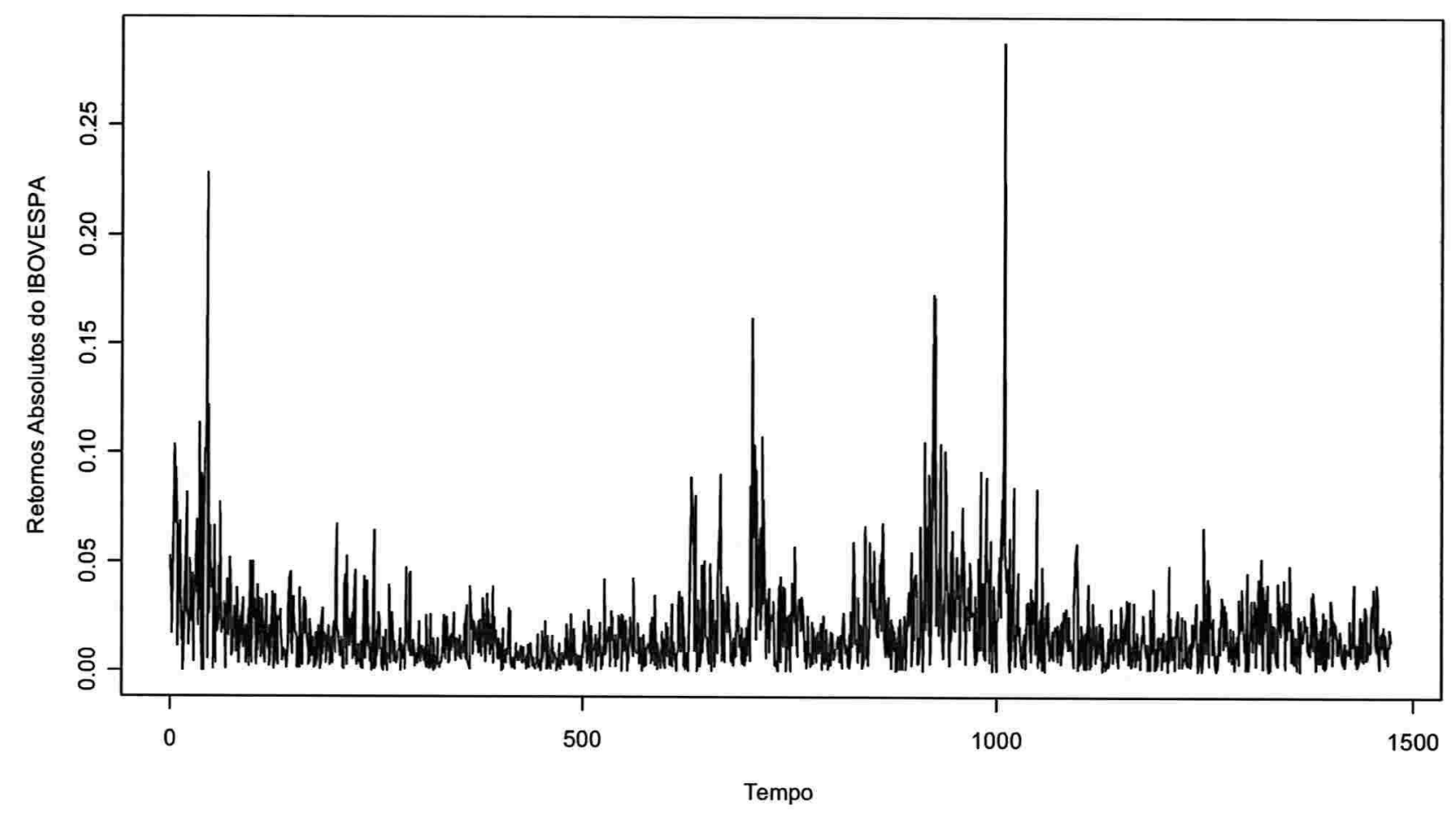

Figura 6.5 - Retornos Absolutos Mensais do IBOVESPA.

Observando sua função de autocorrelação na Figura 6.13, nota-se que ela decai a uma taxa hiperbólica, indicando uma presença possível de memória longa. A estatística de teste R/S é igual a 2,4639, rejeitando a hipótese nula de que não há memória longa a um nível de $1 \%$ de significância. O Quadro 6.6 mostra a saída do software S-PLUS, do teste R/S de memória longa, usando o comando "rosTest", do módulo FinMetrics (ver Anexo 1.1). 
Test for Long Memory: Modified R/S Test

Null Hypothesis: no long-term dependence

Test Statistics:

\subsection{9 **}

* : significant at $5 \%$ level

** : significant at $1 \%$ level

Quadro 6.6 - Saída do teste R/S de memória longa usando o software SPLUS.

Fazendo o teste GPH, também rejeitamos a hipótese nula de que não há memória longa a um nível de $1 \%$ de significância, com uma estatística de teste igual a 3,9181 e um $\hat{d}=0,4752$. A Figura 6.12 mostra a saída do software S-PLUS, do teste GPH de memória longa, usando o comando "gphTest", do módulo FinMetrics (ver Anexo 1.1).

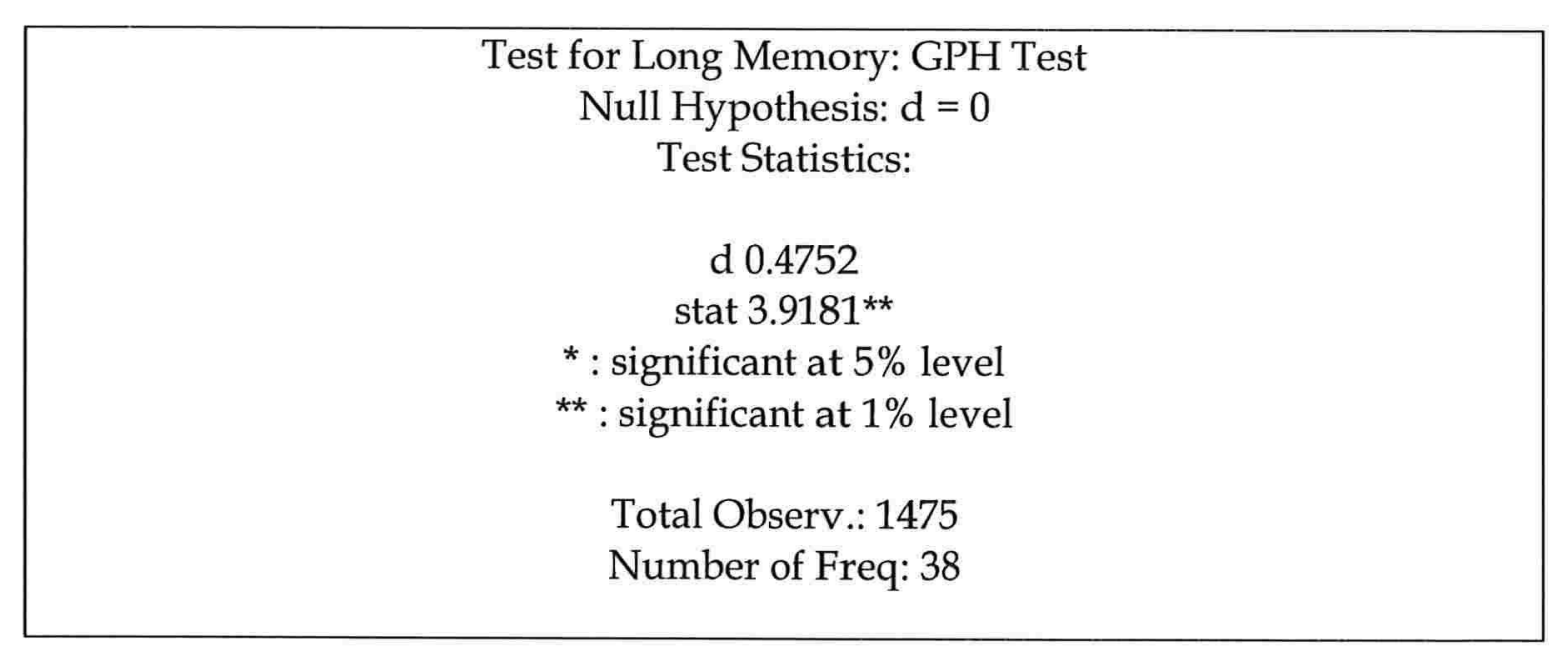

Quadro 6.7 - Saída do teste de GPH de memória longa usando o software S-PLUS. 


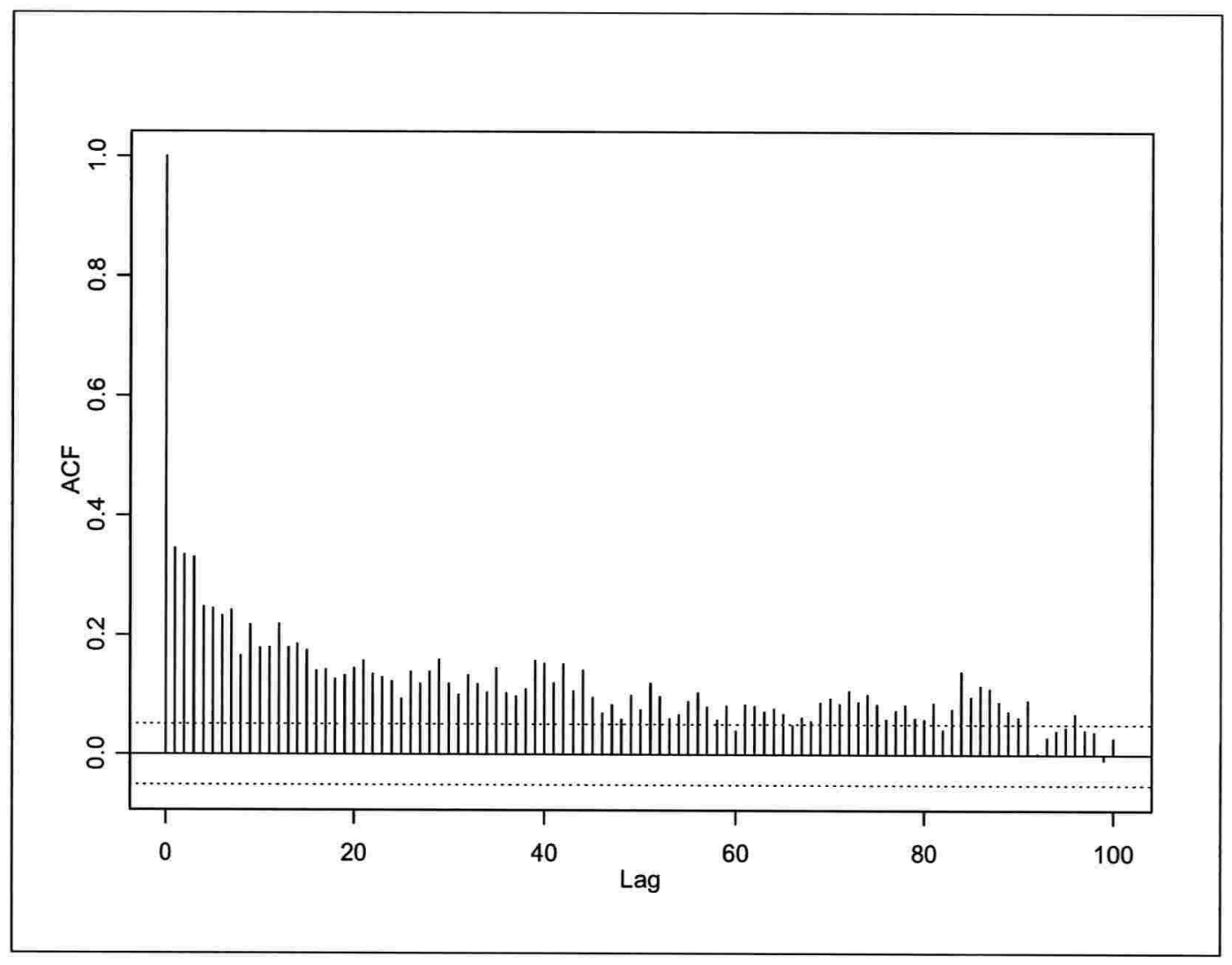

Figura 6.6 - Função de autocorrelação dos retornos absolutos do IBOVESPA.

Seguindo a especificação do FI-STAR delineada no Capítulo 5, começamos especificando um modelo ARFI para a série. Permitimos até a máxima ordem autoregressiva $\mathrm{p}=18$. O BIC indica que um modelo ARFI com $\mathrm{p}=1$ é adequado para essa série. O Quadro 6.8 mostra a saída do software S-PLUS, da estimação de máxima verossimilhança de um modelo ARFI(1,d), usando o comando "FARIMA", do módulo FinMetrics (ver Anexo 1.2). 


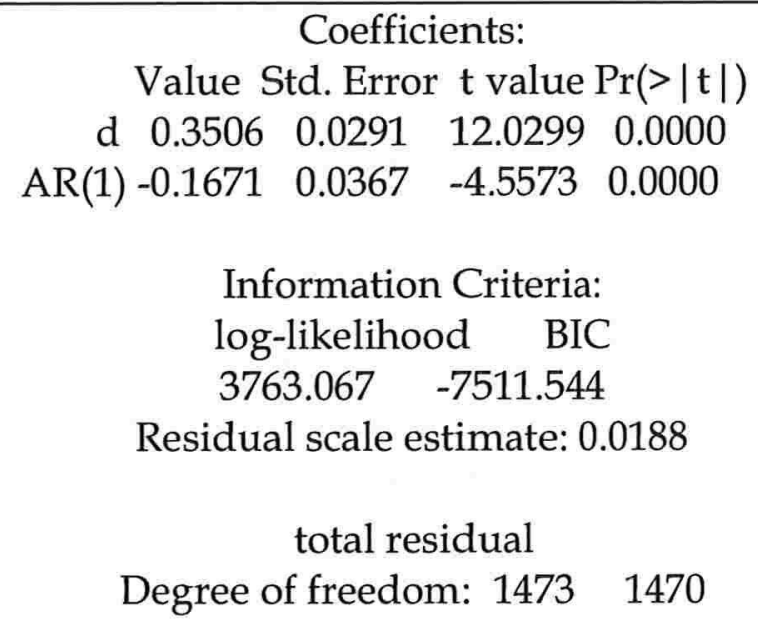

Quadro 6.8 - Saída de um ARFI(1,d) usando o software S-PLUS.

Fizemos o teste de não-linearidade nos resíduos do modelo ARFI(1,d). Como podemos notar na Tabela 6.5, rejeitamos a hipótese nula de linearidade a um nível de $5 \%$ de significância, e $y_{t}$ é a variável de transição escolhida, pois possui uma maior estatística de teste LM.

\begin{tabular}{|c|c|c|}
\hline Variável de Transição & $\begin{array}{c}\text { P-valores da Estatística de } \\
\text { LM F }\end{array}$ & $\begin{array}{c}\text { P-valores da Estatística de } \\
\text { LM } \chi^{2}\end{array}$ \\
\hline$y_{t}$ & 0,00 & $1,33 \mathrm{E}-93$ \\
\hline$y_{t-1}$ & 0,00 & $1,32 \mathrm{E}-03$ \\
\hline$y_{t-2}$ & 0,00 & $4,74 \mathrm{E}-01$ \\
\hline$y_{t-3}$ & 0,00 & $1,00 \mathrm{E}+00$ \\
\hline$y_{t-4}$ & 0,00 & $2,23 \mathrm{E}-04$ \\
\hline$y_{t-5}$ & 0,00 & $1,62 \mathrm{E}-01$ \\
\hline$y_{t-6}$ & 0,00 & $1,35 \mathrm{E}-01$ \\
\hline
\end{tabular}

Tabela 6.5 - Testes LM de linearidade.

Para fazer a estimação do FI-STAR, adotamos o algoritmo de estimação de van Djik (2002), descrito no Capítulo 5 e descrito na aplicação da seção 6.2.1. O Quadro 6.9 mostra a saída do software $R$, da estimação de máxima verossimilhança de um 
modelo STAR aplicado à série de retornos absolutos do IBOVESPA diferenciada fracionariamente, usando a library "tsDyn" e o comando "Istar" (ver Anexo 1.3).

\begin{tabular}{|c|}
\hline Non linear autoregressive model \\
\hline LSTAR model \\
\hline Coefficients: \\
\hline Low regime: \\
\hline phi1.0 phi1.1 \\
\hline$-0.001524485-0.242454813$ \\
\hline High regime: \\
\hline phi2.0 phi2.1 \\
\hline $0.02640213-0.46501889$ \\
\hline Smoothing parameter: gamma $=86.03$ \\
\hline Threshold \\
\hline Variable: $Z(t)=+(1) X(t)$ \\
\hline Value: 0.03968 \\
\hline Residuals: \\
\hline Min $1 Q$ Median $3 Q \quad$ Max \\
\hline$-0.0605248-0.0099366-0.00315770 .00661790 .2307719$ \\
\hline Fit: \\
\hline residuals variance $=0.0003504$, AIC $=-11716, \mathrm{MAPE}=129.7 \%$ \\
\hline Coefficient(s): \\
\hline Estimate Std. Error $t$ value $\operatorname{Pr}(>|t|)$ \\
\hline phi1.0 $-0.00152450 .0029678-0.51370 .607477$ \\
\hline phi1.1 -0.2424548 $0.1202286-2.01660 .043736$ * \\
\hline phi2.0 $0.0264021 \quad 0.01121092 .35500 .018521$ * \\
\hline phi2.1 $-0.46501890 .1045976-4.44588 .757 \mathrm{e}-06$ *** \\
\hline gamma $86.032773563 .6594584 \quad 1.35150 .176550$ \\
\hline th $0.03967960 .01283603 .09130 .001993^{* *}$ \\
\hline Signif. codes: $00^{1 * \star * \mid} 0.001^{1 * * 1} 0.01^{1 * 1} 0.05^{\prime} .^{\prime} 0.1^{\prime \prime} 1$ \\
\hline Non-linearity test of full-order LSTAR model against full-order AR model \\
\hline $\mathrm{F}=4.0879 ; \mathrm{p}$-value $=0.043372$ \\
\hline Threshold \\
\hline Variable: $Z(t)=+(1) X(t)$ \\
\hline
\end{tabular}

Quadro 6.9 - Saída da estimação de um modelo STAR feita usando o software R. 
A Tabela 6.6 mostra os coeficientes estimados pelo FI-STAR ajustado à série de retornos absolutos do IBOVESPA. Na Figura 6.7, comparamos o FI-STAR estimado com a série original diferenciada fracionariamente. Nota-se que o modelo FI-STAR ajusta-se bem a essa série. O parâmetro de suavidade $\hat{\gamma}$ é muito grande, indicando que o modelo FI-STAR se aproxima de um FI-TAR, com uma mudança abrupta de regime.

Fizemos o teste de não-linearidade restante, cujos resultados são mostrados na Tabela 6.7, e não rejeitamos a hipótese nula de que o modelo está corretamente especificado. Note que a não-significância de $\hat{\gamma}$ não deve ser levada em conta, pois, como vimos no Capítulo 4, a estimativa de $\gamma$ em geral é imprecisa e aparece insignificante pela estatística $t$.

\begin{tabular}{ccccc}
\hline Coeficientes & Estimativa & Erro Padrão & Estatística t & P-Valor \\
\hline$\phi_{0,1}$ & $-0,002$ & 0,003 & $-0,514$ & 0,607 \\
$\phi_{1,1}$ & $-0,242$ & 0,120 & $-2,017$ & 0,044 \\
$\phi_{0,2}$ & 0,026 & 0,011 & 2,355 & 0,019 \\
$\phi_{1,2}$ & $-0,465$ & 0,105 & $-4,446$ & 0,000 \\
$\hat{\gamma}$ & 86,033 & 63,659 & 1,352 & 0,177 \\
$\hat{c}$ & 0,040 & 0,013 & 3,091 & 0,002 \\
$\hat{d}$ & 0,351 & 0,029 & 12,030 & 0,000 \\
\hline
\end{tabular}

Tabela 6.6 - Coeficientes estimados pelo FI-STAR. 


\begin{tabular}{|c|c|}
\hline Variável de Transição & P-valores da Estatística de LM $\chi^{2}$ \\
\hline$y_{t}$ & 0,045 \\
\hline$y_{t-1}$ & 0,400 \\
\hline$y_{t-2}$ & 0,289 \\
\hline$y_{t-3}$ & 0,034 \\
\hline$y_{t-4}$ & 0,117 \\
\hline$y_{t-5}$ & 0,709 \\
\hline$y_{t-6}$ & 0,776 \\
\hline
\end{tabular}

Tabela 6.7 - Teste LM $\chi^{2}$ de não-linearidade restante.

De acordo com a Tabela 6.6, o modelo ajustado é dado por:

$$
\begin{aligned}
& (1-L)^{\hat{d}} y_{t}=x_{t}, \hat{\mathrm{d}}=0,351 \\
& (0,029) \\
& x_{t}=\left(-0,002-0,242 x_{t-1}\right) \times\left(1-G\left(y_{t} ; \hat{\gamma} ; \hat{c}\right)\right)+\left(0,026-0,465 x_{t-1}\right) \times G\left(y_{t} ; \hat{\gamma} ; \hat{c}\right), \\
& (0,003)(0,120)
\end{aligned}
$$

$G\left(y_{t} ; \hat{\gamma} ; \hat{c}\right)=\left(1+\exp \left\{-86,033\left(y_{t}-0,040\right)\right\}\right)^{-1}$

$$
(63,659) \quad(0,013)
$$

$\hat{\sigma}^{2}=3.50 \mathrm{E}-04$ 


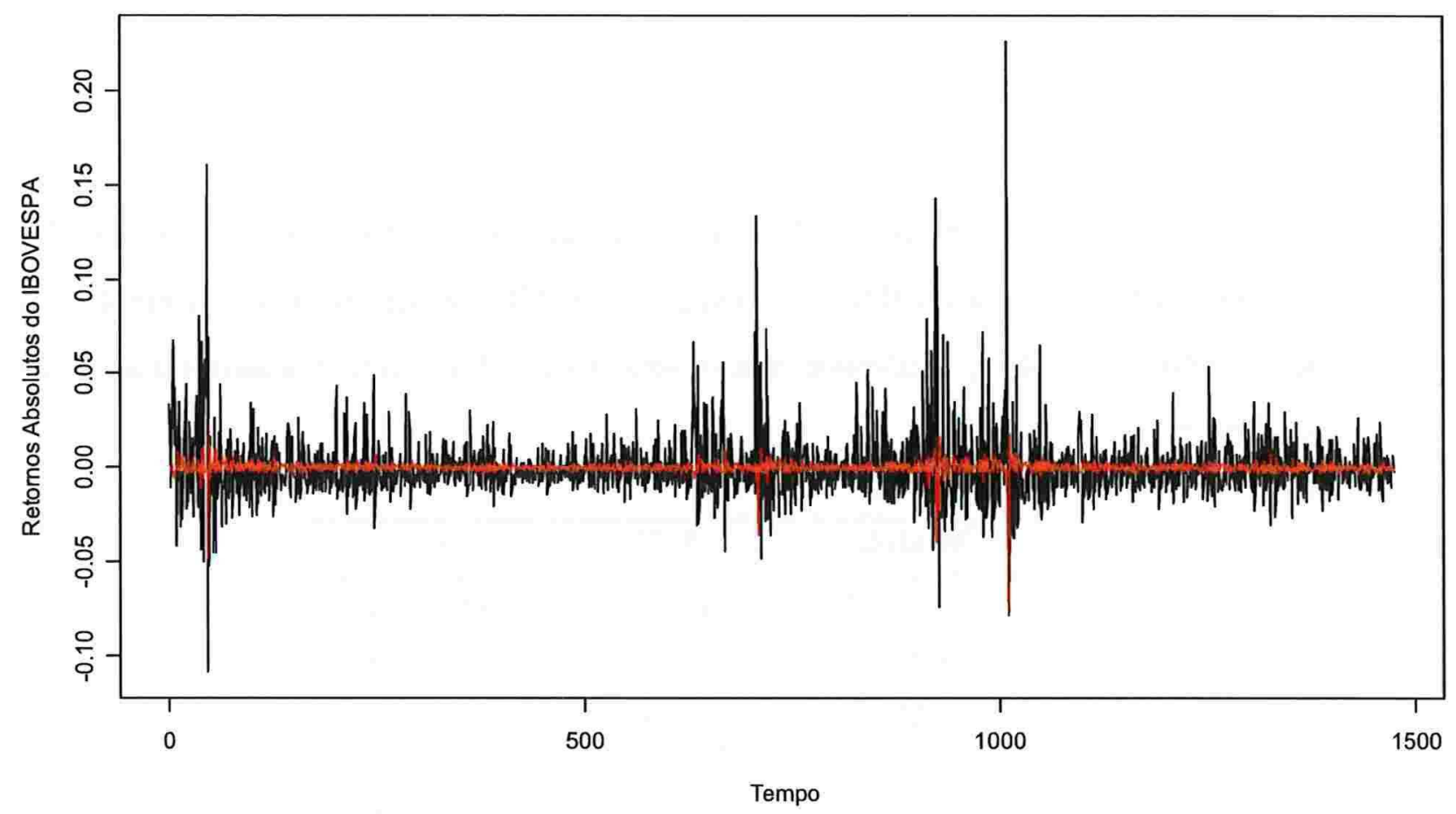

Figura 6.7 - Retornos absolutos do IBOVESPA diferenciado fracionariamente (--) e série ajustada (--).

Para efeito de comparação, também ajustamos os modelos:

(i) FI-TAR:

$(1-L)^{\hat{d}} y_{t}=x_{t}, \hat{\mathrm{d}}=0,351$

$(0,029)$

$x_{t}=\left(-0,001-0,211 x_{t-1}\right) \times\left(1-I\left(y_{t}>0,013\right)\right)+\left(0,005-0,261 x_{t-1}\right) \times I\left(y_{t}>0,013\right)$,

$(0,001)(0,048) \quad(0,002)(0,051)$

$\hat{\sigma}^{2}=3.51 \mathrm{E}-04$. 
(ii) AR:

$y_{t}=0,019+0,205 y_{t-1}+0,182 y_{t-2}+0,182 y_{t-3}+0,056 y_{t-4}$

$(0,001)(0,026) \quad(0,026) \quad(0,026) \quad(0,026)$

$\hat{\sigma}^{2}=3.60 \mathrm{E}-04$.

A Tabela 6.8 relaciona o AIC e a variância dos resíduos para cada um dos modelos ajustados. Para calcular e comparar o AIC desses modelos, controlamos novamente a amostra, e usamos a amostra do modelo com o maior número de parâmetros.

\begin{tabular}{ccc}
\hline Modelo & AIC & $\hat{\sigma}^{2}$ \\
\hline FI-STAR & -11.684 & $3,50 \mathrm{E}-04$ \\
FI-TAR & -11.681 & $3,51 \mathrm{E}-04$ \\
AR & -11.648 & $3,60 \mathrm{E}-04$ \\
\hline
\end{tabular}

Tabela 6.8 - Coeficientes estimados pelo FI-STAR.

Como podemos notar na Tabela 6.8, a variância dos resíduos é muito próxima para os três modelos, entretanto o FI-STAR e o FI-TAR possuem um ajuste melhor em termos do critério de AIC. Como observamos anteriormente, o fato de $\hat{\gamma}$ ser muito grande justifica que o ajuste do modelo FI-STAR se aproxime de um FI-TAR.

\subsection{Aplicação do FI-BREAK}

\subsubsection{Retornos Mensais da Treasure-Bill (T-Bill) Americana}

Considere a série de retornos mensais da T-Bill americana, de janeiro de 1934 a dezembro de 2003, apresentada na Figura 6.8 abaixo. Ao todo, temos 840 observações. 


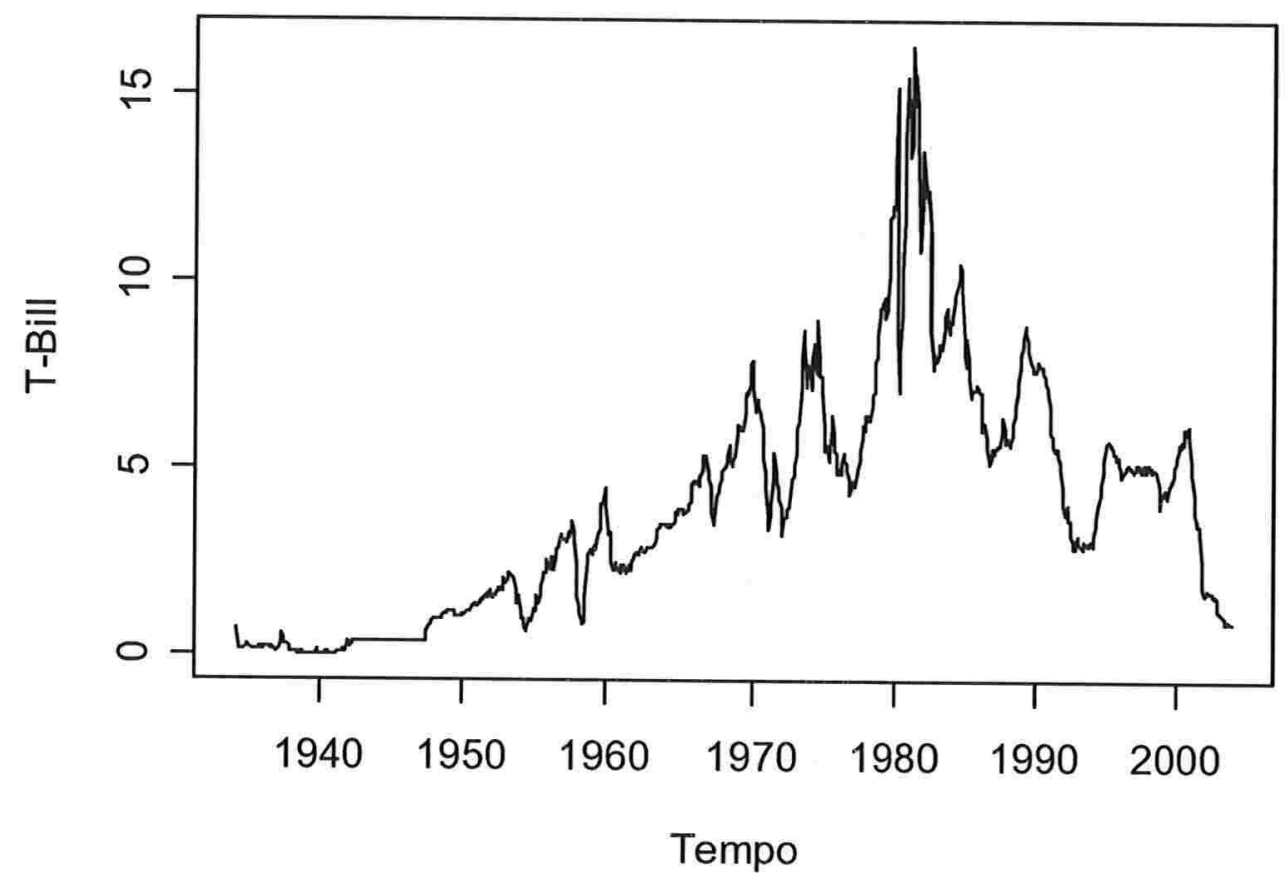

Figura 6.8 - Retornos mensais da T-Bill americana.

Olhando para a função de auto-correlação na Figura 6.9, vemos que ela não decai para zero. A estatística de teste R/S obtida é igual a 4,371, rejeitando a hipótese nula de que não há memória longa a um nível de $1 \%$ de significância. $\mathrm{O}$ Quadro 6.10 mostra uma saída do software S-PLUS, do teste R/S de memória longa.

\section{Test for Long Memory: Modified R/S Test \\ Null Hypothesis: no long-term dependence Test Statistics:$$
4.317^{* *}
$$ \\ * : significant at $5 \%$ level \\ ** : significant at $1 \%$ level}

Quadro 6.10 - Saída do teste R/S de memória longa usando o software SPLUS 
Fazendo o teste GPH, também rejeitamos a hipótese nula de que não há memória longa a um nível de $1 \%$ de significância, com uma estatística de teste igual a 6,4661 e um $\hat{d}=0,9455$. O Quadro 6.11 mostra uma saída do software SPLUS, do teste GPH de memória longa.

\begin{tabular}{|c|}
\hline Test for Long Memory: GPH Test \\
Null Hypothesis: $d=0$ \\
Test Statistics: \\
d 0.9455 \\
stat $6.4661^{* *}$ \\
* : significant at $5 \%$ level \\
** : significant at $1 \%$ level \\
Total Observ.: 426 \\
Number of Freq: 20 \\
\hline
\end{tabular}

Quadro 6.11 - Saída do teste GPH de memória longa usando o software S-PLUS.

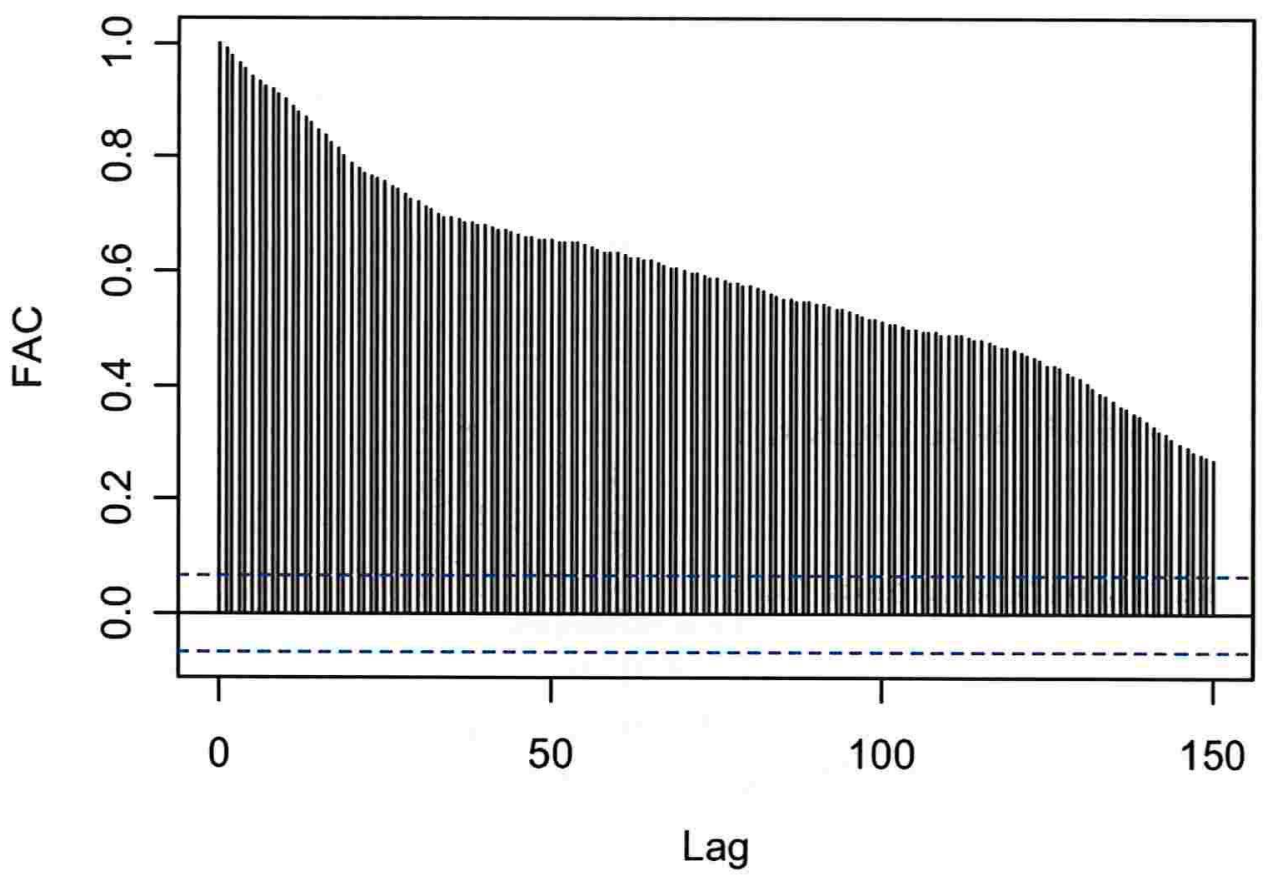

Figura 6.9 - Função de auto-correlação dos retornos mensais da T-Bill americana. 
Assim, podemos ajustar um modelo de memória longa para essa série. Vamos verificar, usando o algoritmo de Bai \& Perron (2003), se a série possui quebras estruturais. Como podemos notar na Figura 6.10 e na Tabela 6.9 abaixo, aplicando esse algoritmo, o critério de BIC e a soma dos quadrados dos resíduos são minimizados com 5 quebras estruturais na série. Portanto, podemos concluir que a série possui tanto memória longa como quebras estruturais, sugerindo a utilização do FI-BREAK.

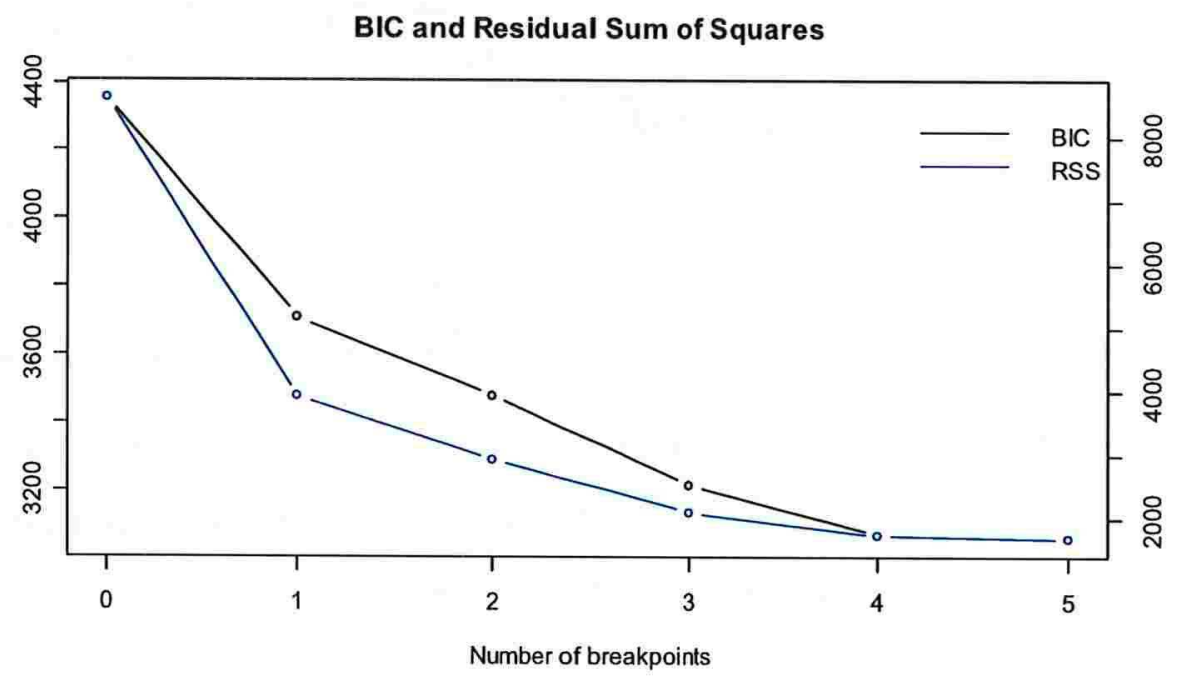

Figura 6.10 - Número de quebras estruturais X BIC e soma dos quadrados dos resíduos.

\begin{tabular}{|c|c|c|c|c|c|c|}
\hline Número de Quebras & 0 & 1 & 2 & 3 & 4 & 5 \\
\hline SQR & 8.627 & 3.929 & 2.943 & 2.118 & 1.752 & 1.701 \\
\hline BIC & 4.354 & 3.707 & 3.477 & 3.215 & 3.069 & 3.057 \\
\hline
\end{tabular}

Tabela 6.9 - Número de quebras estruturais X BIC e soma dos quadrados dos resíduos. 
A Figura 6.11 abaixo mostra a série de retornos mensais da T-Bill americana, com uma média diferente para cada um dos segmentos entre as quebras estruturais detectadas pelo algoritmo de Bai \& Perron (2003).

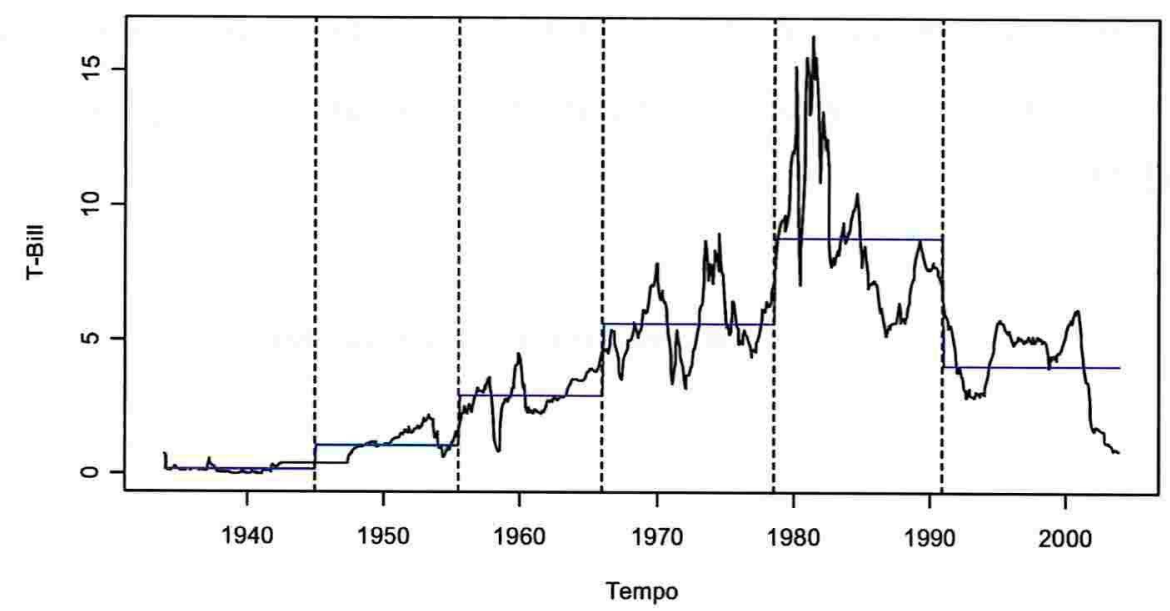

Figura 6.11 - Retornos mensais da T-Bill americana e quebras estruturais.

O Quadro 6.12 mostra uma saída do software RATS, de uma estimação de máxima verossimilhança aproximada de um FI-BREAK, usando o comando "maximize" e o método BHHH (ver Anexo 1.5).

MAXIMIZE - Estimation by BHHH

Convergence in 1 Iterations. Final criterion was $0.0000000<0.0000100$

Monthly Data From 1934:07 To 2003:01

Usable Observations 823

Function Value $\quad 707.29451321$

Variable Coeff Std Error T-Stat Signif

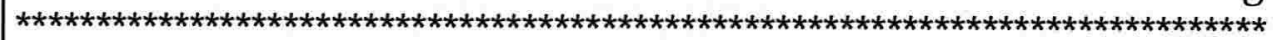

$\begin{array}{lllll}\text { 1. D } & 0.4207888628 & 0.0000944567 & 4454.83155 & 0.00000000 \\ \text { 2. GAMMA } & 0.0026581308 & 0.0000224551 & 118.37513 & 0.00000000 \\ \text { 3. VAR } & 0.1662967439 & 0.0011392549 & 145.96974 & 0.00000000\end{array}$

Quadro 6.12 - Saída de um FI-BREAK usando o software RATS.

Estimamos um FI-BREAK na seguinte forma: 


$$
\begin{aligned}
& \begin{array}{l}
(1-L)^{\hat{d}} y_{t}=m_{t}+\varepsilon_{t}, \hat{\mathrm{d}}=0,421 \\
(9,45 \mathrm{E}-05)
\end{array} \\
& m_{t}=m_{t-1}+q_{t-1} \varepsilon_{t-1}, \\
& q_{t-1}=\frac{\left(\varepsilon_{t-1}+\varepsilon_{t-2}+\varepsilon_{t-3}+\varepsilon_{t-4}+\varepsilon_{t-5}+\varepsilon_{t-6}\right)^{2}}{0,00266+\left(\varepsilon_{t-1}+\varepsilon_{t-2}+\varepsilon_{t-3}+\varepsilon_{t-4}+\varepsilon_{t-5}+\varepsilon_{t-6}\right)^{2}} \\
& \quad(2,25 \mathrm{E}-05) \\
& \hat{\sigma}^{2}=0,1663 .
\end{aligned}
$$

\begin{tabular}{ccccc}
\hline Coeficientes & Estimativa & Erro Padrão & Estatística $\mathbf{~}$ & P-Valor \\
\hline$\hat{d}$ & 0,421 & $9,45 \mathrm{E}-05$ & 4454,878 & 0,00 \\
$\hat{\gamma}$ & $2,66 \mathrm{E}-03$ & $2,25 \mathrm{E}-05$ & 118,376 & 0,00 \\
\hline
\end{tabular}

Tabela 6.10 - Coeficientes estimados do FI-BREAK.

Como podemos observar no modelo acima, temos um caso em que $\gamma$ é aproximadamente 0. Na Figura 6.12, comparamos o FI-BREAK ajustado com a série original da T-Bill. De acordo com a Tabela 5.1, isso sugere a utilização de modelos ARFI e ARI. 


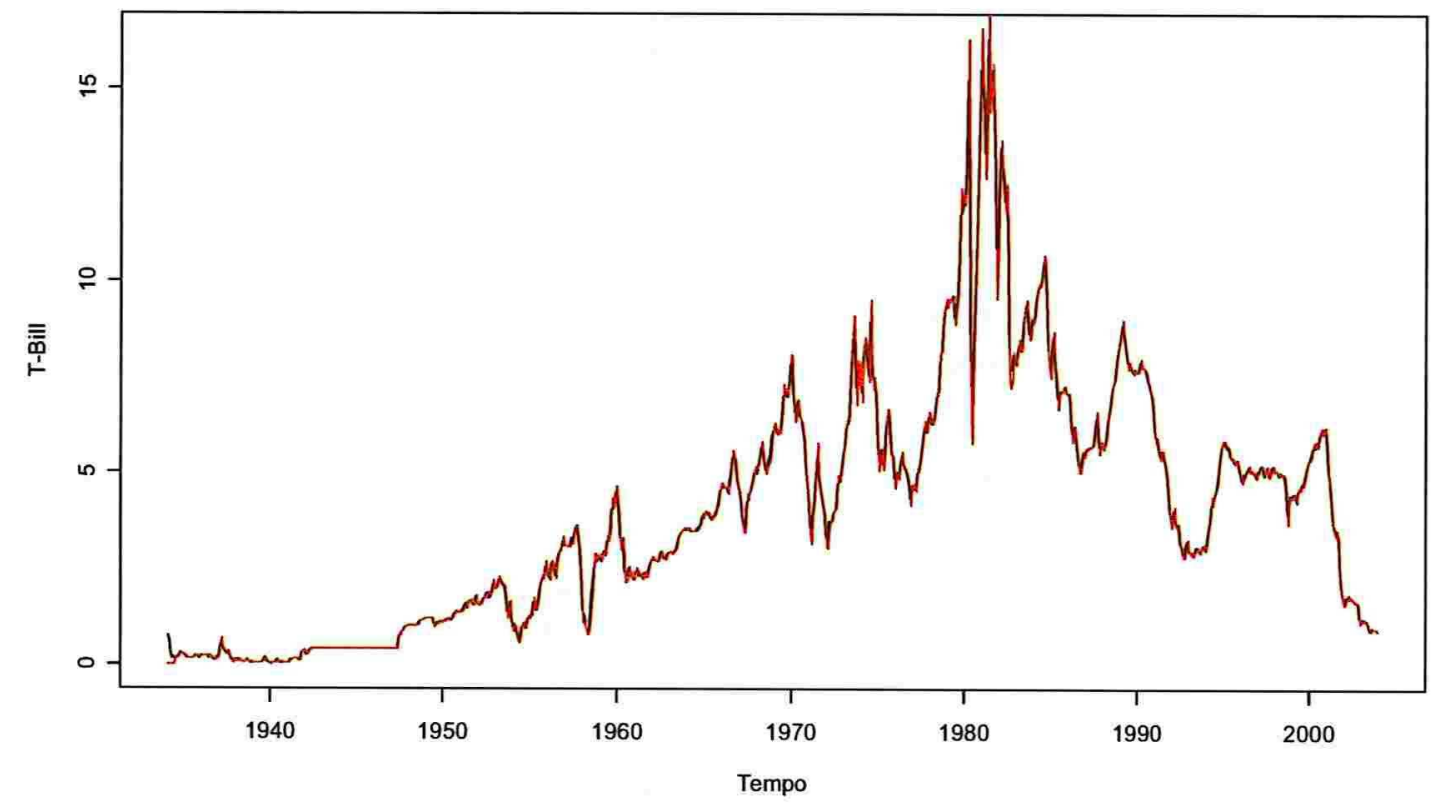

Figura 6.12 - Séries T-Bill original (--) e ajustada utilizando o FI-BREAK (--).

Para efeito de comparação, também ajustamos os modelos:

(i) STOP-BREAK:

$y_{t}=-0,288 y_{t-1}+m_{t}+\varepsilon_{t}$,

$(0,006)$

$m_{t}=m_{t-1}+q_{t-1} \varepsilon_{t-1}$

$q_{t-1}=\frac{\left(\varepsilon_{t-1}+\varepsilon_{t-2}+\varepsilon_{t-3}+\varepsilon_{t-4}+\varepsilon_{t-5}+\varepsilon_{t-6}\right)^{2}}{0,000002+\left(\varepsilon_{t-1}+\varepsilon_{t-2}+\varepsilon_{t-3}+\varepsilon_{t-4}+\varepsilon_{t-5}+\varepsilon_{t-6}\right)^{2}}$

$(4,26 \mathrm{E}-05)$

$\hat{\sigma}^{2}=0,1447$. 
(ii) $\operatorname{ARI}(2,1)$ :

$$
\begin{aligned}
\Delta y_{t}= & 0,001+0,403 \Delta y_{t-1}-0,216 \Delta y_{t-2} \\
& (0,015)(0,034) \quad(0,034) \\
\hat{\sigma}^{2}= & 0,1311 .
\end{aligned}
$$

(iii) $\operatorname{ARFI}(2,1+0,206)$ :

$$
(1-L)^{1+\grave{d}} y_{t}=x_{t}, \hat{\mathrm{d}}=0,206
$$

$$
(4,11 \mathrm{E}-06)
$$

$$
\begin{gathered}
\Delta x_{t}=0,234 \Delta x_{t-1}-0,249 \Delta x_{t-2} \\
(0,033) \quad(0,033)
\end{gathered}
$$

$\hat{\sigma}^{2}=0,1339$.

A Tabela 6.11 relaciona o AIC e a variância dos resíduos para cada um dos modelos ajustados.

\begin{tabular}{ccc}
\hline Modelo & AIC & $\hat{\sigma}^{2}$ \\
\hline FI-BREAK & -1.494 & 0,166 \\
STOP-BREAK & -1.606 & 0,145 \\
ARI & -1.689 & 0,131 \\
ARFI & -1.668 & 0,134 \\
\hline
\end{tabular}

Tabela 6.11 - AIC e variância dos resíduos

Como podemos notar na Tabela 6.11, tanto a menor variância residual como o melhor ajuste em termos do critério de AIC são obtidos usando o modelo ARI $(2,1)$. Isso é coerente com a Tabela 5.1, que sugere modelos ARI e ARFI quando $\gamma \rightarrow 0$. 


\subsection{2 Índice de Preços do Trigo de Beveridge}

Considere a série do índice de preços do trigo de Beveridge, de 1500 a 1845, apresentada na Figura 6.13. Ao todo, temos 346 observações.

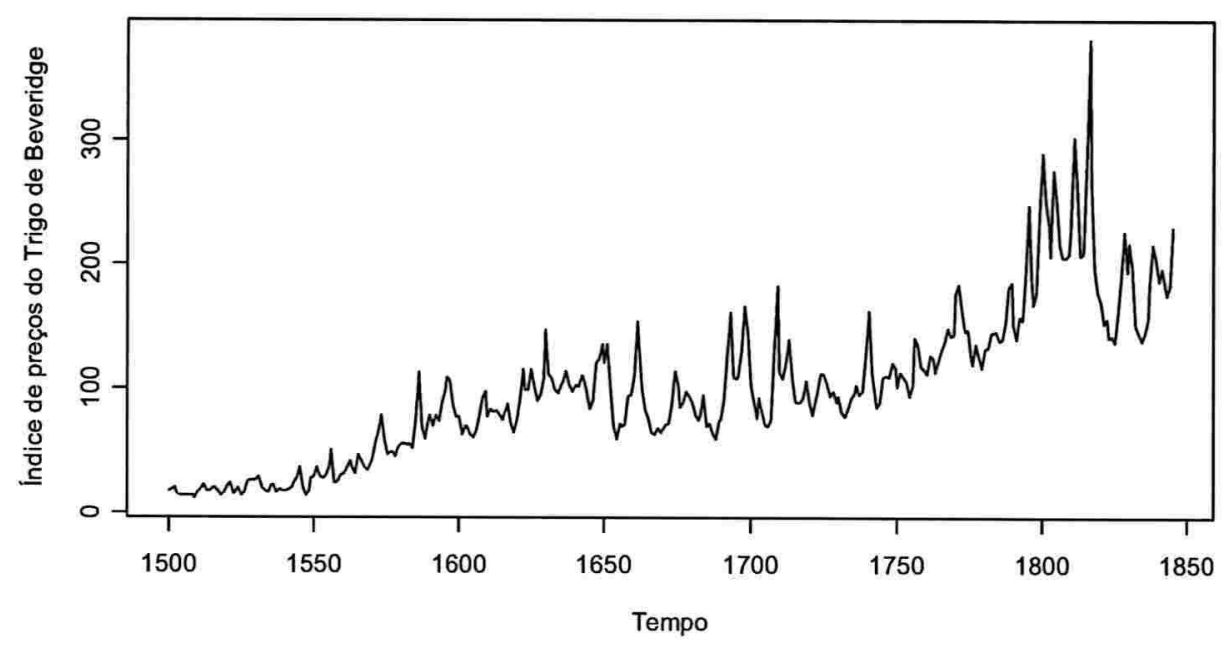

Figura 6.13 - Índice de preços do trigo de Beveridge.

Olhando para sua função de auto-correlação na Figura 6.14, vemos que ela demora em decair, a uma taxa lenta hiperbólica. A estatística de teste R/S obtida é igual a 2,7502, rejeitando a hipótese nula de que não há memória longa a um nível de 1\% de significância. O Quadro 6.13 mostra a saída do software S-PLUS, do teste $\mathrm{R} / \mathrm{S}$ de memória longa.

Test for Long Memory: Modified R/S Test

Null Hypothesis: no long-term dependence Test Statistics:

$2.7502^{\star \star}$

* : significant at $5 \%$ level

** : significant at $1 \%$ level

Quadro 6.13 - Saída do teste R/S de memória longa usando o software SPLUS. 
Fazendo o teste GPH, também rejeitamos a hipótese nula de que não há memória longa a um nível de $1 \%$ de significância, com uma estatística de teste igual 6,3383e um $\hat{d}=1,2312$. O Quadro 6.14 mostra a saída do software S-PLUS, do teste GPH de memória longa.

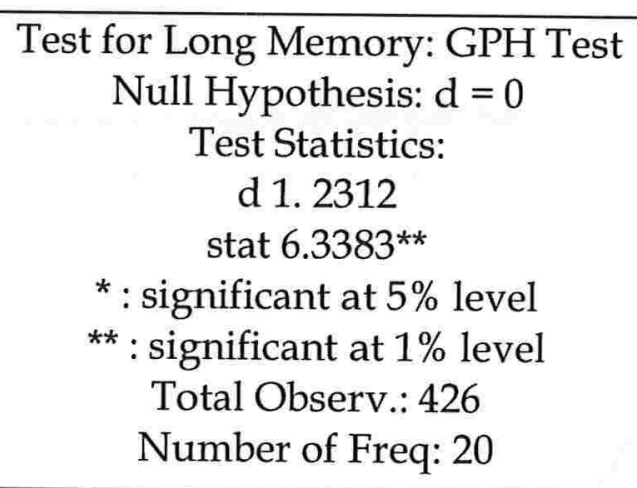

Quadro 6.14 - Saída do teste GPH de memória longa usando o software S-PLUS.

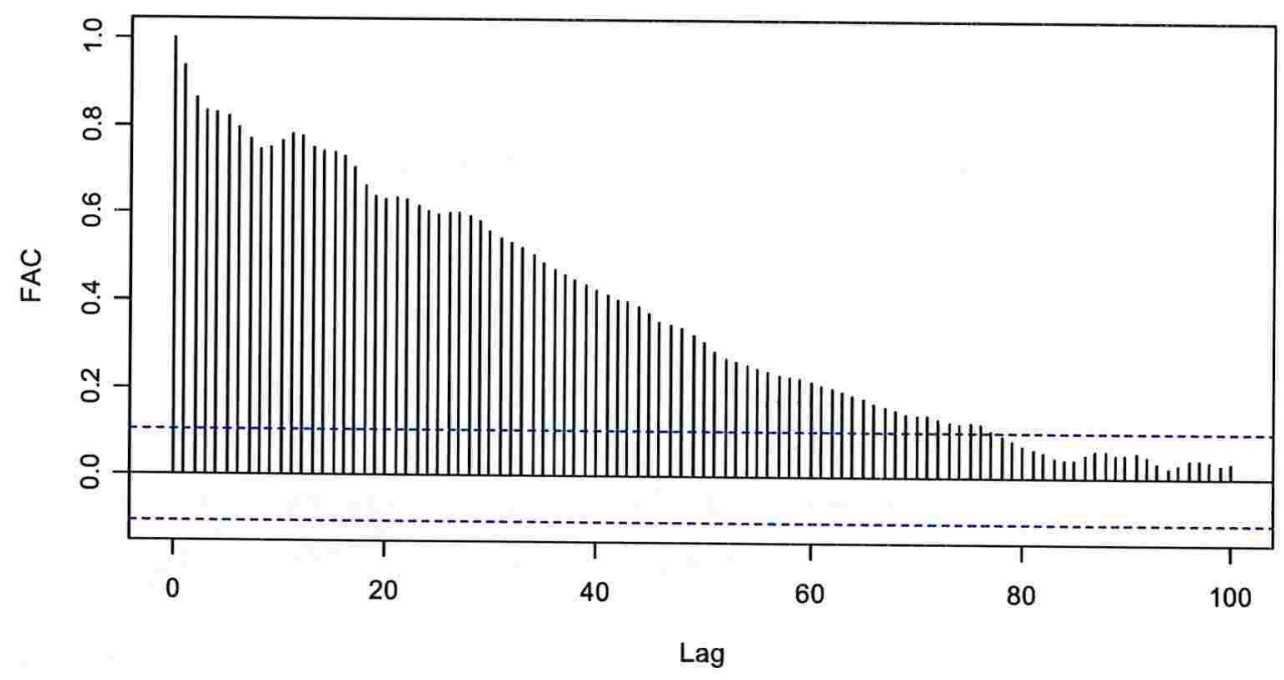

Figura 6.14 - Função de auto-correlação do índice de preços do trigo de Beveridge. 
Assim, podemos ajustar um modelo de memória longa para essa série. Vamos verificar, usando o algoritmo de Bai \& Perron (2003), se a série possui quebras estruturais. Como podemos notar na Figura 6.15 e na Tabela 6.12, aplicando esse algoritmo, o critério de BIC e a soma dos quadrados dos resíduos é minimizada com 4 quebras estruturais. Portanto, a série possui tanto memória longa como quebras estruturais, sugerindo a utilização do FI-BREAK.

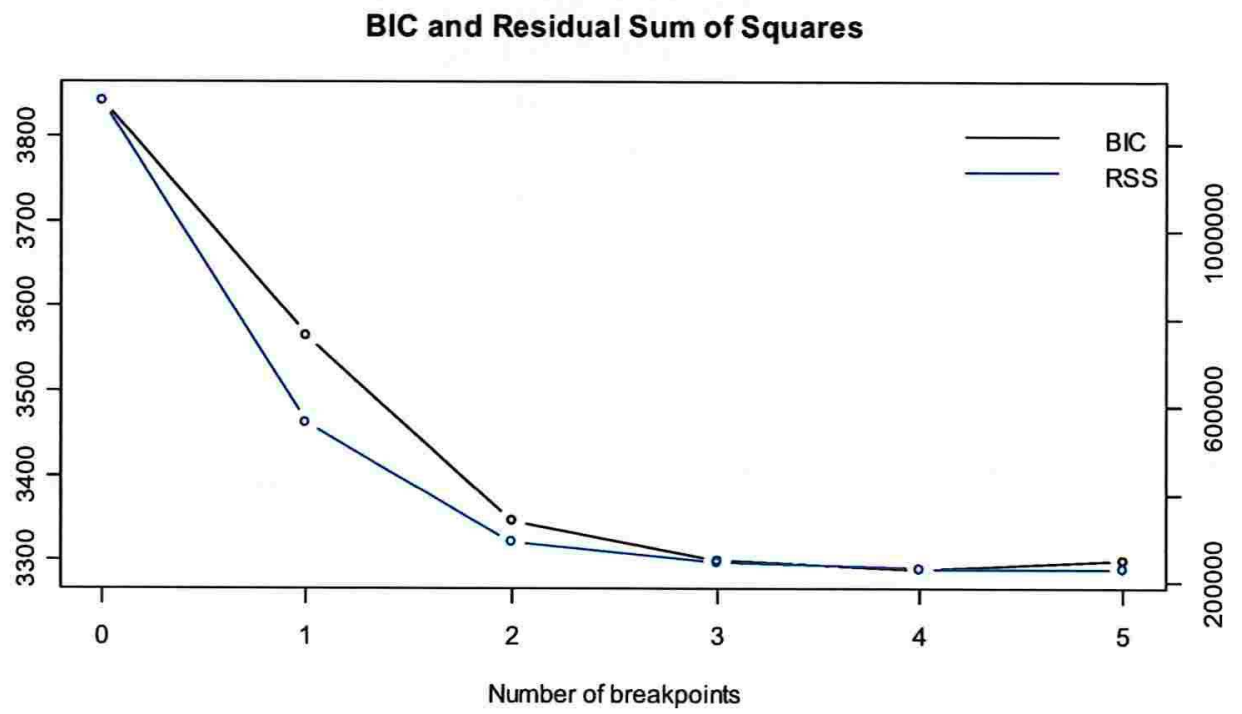

Figura 6.15 - Número de quebras estruturais X BIC e soma dos quadrados dos resíduos.

\begin{tabular}{|c|c|c|c|c|c|c|}
\hline Número de Quebras & 0 & 1 & 2 & 3 & 4 & 5 \\
\hline SQR & 1.299 .963 & 564.735 & 290.643 & 244.970 & 230.170 & 228.749 \\
\hline BIC & 3.842 & 3.565 & 3.347 & 3.299 & 3.289 & 3.299 \\
\hline
\end{tabular}

Tabela 6.12 - Número de quebras estruturais X BIC e soma dos quadrados dos resíduos.

A Figura 6.16 mostra a série do índice de preços do trigo de Beveridge, com uma média diferente para cada um dos segmentos. 


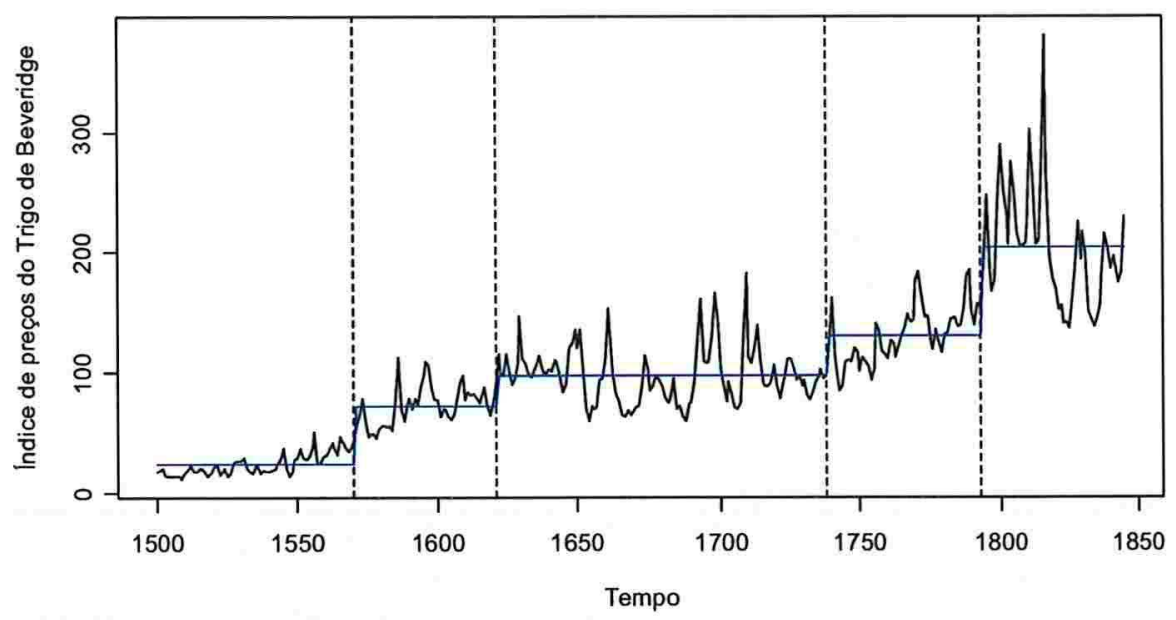

Figura 6.16 - Índice de preços do trigo de Beveridge e quebras estruturais.

O Quadro 6.15 mostra a saída do software RATS, de uma estimação de máxima verossimilhança aproximada de um FI-BREAK, usando o comando "maximize" e o método BHHH (ver Anexo 1.5).

\section{MAXIMIZE - Estimation by BHHH}

Convergence in 1 Iterations. Final criterion was $0.0000000<0.0000100$

Annual Data From 1507:01 To 1845:01

Usable Observations 339

Function Value

$-2433.50349123$

Variable Coeff Std Error T-Stat Signif

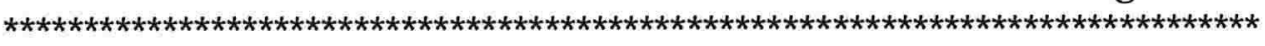

$\begin{array}{lllll}\text { 1. D } & 0.13686193 & 0.00335495 & 40.79400 & 0.00000000\end{array}$

$\begin{array}{lllll}\text { 2. GAMMA } & 6.80875999 & 0.20731838 & 32.84205 & 0.00000000\end{array}$

$\begin{array}{lllll}\text { 3. VAR } & 482.18521563 & 9.32585296 & 51.70414 & 0.00000000\end{array}$

Quadro 6.15 - Saída de um FI-BREAK usando o software RATS.

O modelo FI-BREAK estimado é dado por: 


$$
\begin{aligned}
& \begin{aligned}
\begin{aligned}
(1-L)^{\hat{d}} y_{t}=m_{t}+\varepsilon_{t}, \hat{\mathrm{d}}=0,137 \\
(0,003)
\end{aligned} \\
m_{t}=m_{t-1}+q_{t-1} \varepsilon_{t-1}, \\
q_{t-1}=\frac{\left(\varepsilon_{t-1}+\varepsilon_{t-2}+\varepsilon_{t-3}+\varepsilon_{t-4}+\varepsilon_{t-5}+\varepsilon_{t-6}\right)^{2}}{6,80876+\left(\varepsilon_{t-1}+\varepsilon_{t-2}+\varepsilon_{t-3}+\varepsilon_{t-4}+\varepsilon_{t-5}+\varepsilon_{t-6}\right)^{2}} . \\
(0,207)
\end{aligned} \\
& \hat{\sigma}^{2}=482,19 .
\end{aligned}
$$

\begin{tabular}{ccccc}
\hline Coeficientes & Estimativa & Erro Padrão & Estatística t & P-Valor \\
\hline$\hat{d}$ & 0,137 & 0,003 & 40,794 & 0,000 \\
$\hat{\gamma}$ & 6,809 & 0,207 & 32,842 & 0,000 \\
\hline
\end{tabular}

Tabela 6.13 - Coeficientes estimados pelo FI-BREAK.

Como podemos observar no modelo acima, temos um caso em que $0<\mathrm{d}<1$ e $0<\gamma<\infty$. De acordo com a Tabela 5.1, isso sugere a utilização de um modelo FIBREAK. Na Figura 6.17, comparamos o FI-BREAK ajustado com a série original.

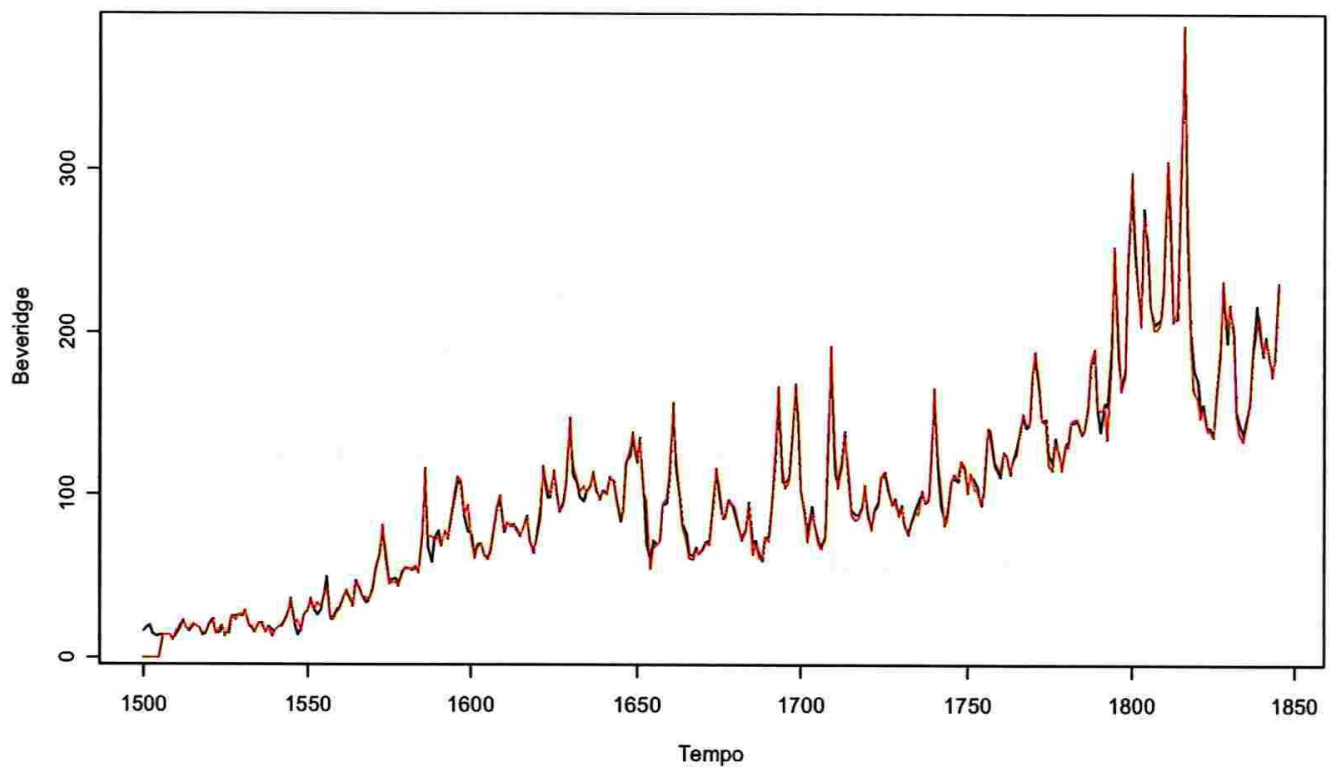

Figura 6.17 - Séries do índice de preços do trigo de Beveridge real (--) e ajustada (--). 
Para efeito de comparação, também ajustamos os modelos:

(i) STOP-BREAK:

$y_{t}=m_{t}+\varepsilon_{t}$,

$m_{t}=m_{t-1}+q_{t-1} \varepsilon_{t-1}$,

$q_{t-1}=\frac{\left(\varepsilon_{t-1}+\varepsilon_{t-2}+\varepsilon_{t-3}+\varepsilon_{t-4}+\varepsilon_{t-5}+\varepsilon_{t-6}\right)^{2}}{18,03+\left(\varepsilon_{t-1}+\varepsilon_{t-2}+\varepsilon_{t-3}+\varepsilon_{t-4}+\varepsilon_{t-5}+\varepsilon_{t-6}\right)^{2}}$

$(1,100)$

$\hat{\sigma}^{2}=470,68$.

(ii) $\operatorname{ARI}(3,1)$ :

$\begin{aligned} \Delta y_{t}= & 0,567+0,018 \Delta y_{t-1}-0,326 \Delta y_{t-2}-0,208 \Delta y_{t-3} \\ & (0,676)(0,053) \quad(0,050) \quad(0,053)\end{aligned}$

$\hat{\sigma}^{2}=361,10$

(iii) $\operatorname{ARFI}(2,0.532)$ :

$(1-L)^{\hat{d}} y_{t}=x_{t}, \hat{\mathrm{d}}=0,532$

$(0,075)$

$\Delta x_{t}=0,485 \Delta x_{t-1}-0,245 \Delta x_{t-2}$

$(0,054) \quad(0,054)$

$\hat{\sigma}^{2}=381,9$.

A Tabela 6.14 relaciona o critério de AIC e a variância dos resíduos para cada um dos modelos ajustados. 


\begin{tabular}{ccc}
\hline Modelo & AIC & $\hat{\sigma}^{2}$ \\
\hline FI-BREAK & 2.105 & 482,19 \\
STOP-BREAK & 2.094 & 470,68 \\
ARI & 2.010 & 361,10 \\
ARFI & 2.029 & 381,90 \\
\hline
\end{tabular}

Tabela 6.14 - Coeficientes estimados pelo FI-BREAK.

Como podemos notar na Tabela 6.14, tanto a menor variância residual como o melhor ajuste em termos do critério de AIC são obtidos usando o modelo ARI(3,1). 


\section{Capítulo 7}

\section{Conclusões}

Neste trabalho exploramos a possibilidade de que o modelo de quebra estrutural (ou modelo BREAK) e o processo $\operatorname{ARFIMA}(\mathrm{p}, \mathrm{d}, \mathrm{q})$ de memória longa possam ser resumidos em um único modelo, o modelo FI-BREAK; tentamos também capturar a característica de mudança de regime (modelo STAR) e o processo $\operatorname{ARFIMA}(\mathrm{p}, \mathrm{d}, \mathrm{q})$ de memória longa em um único modelo, o modelo FISTAR.

Para a série da taxa de desemprego norte-americana dessasonalizada, de janeiro de 1969 a junho de 2004, o modelo FI-STAR obteve um ajuste melhor para essa série do que o modelo auto-regressivo ou aqueles que incluem apenas mudança de regime, como o modelo STAR. Para a série de retornos absolutos diários do IBOVESPA, de 03.01.1995 a 22.11.2000, o modelo FI-STAR possui um ajuste melhor em termos do critério de AIC do que um modelo auto-regressivo com muitos parâmetros. Dessa forma, o modelo FI-STAR, para as séries estudadas, mostrou ser um modelo parcimonioso e com um bom ajuste para séries com memória longa e mudança de regime.

Para a série de retornos mensais da T-Bill americana, de janeiro de 1934 a dezembro de 2003, o modelo FI-BREAK não obteve um ajuste melhor do que outros modelos, como o modelo ARI $(2,1)$ e ARFI. Porém, o FI-BREAK possui a vantagem de agrupar quatro modelos possíveis em um só, pois, de acordo com a 
Tabela 5.1, quando $\gamma \rightarrow 0$, sugere-se a utilização de modelos ARI e ARFI, como foi o caso da série da T-Bill.

Para a série do índice de preços do trigo de Beveridge, de 1500 a 1845, os modelos FI-BREAK e o STOP-BREAK não possuem um ajuste melhor em termos do critério de AIC do que um modelo auto-regressivo. Dessa forma, o modelo FIBREAK parece, para as séries analisadas, ser um guia de ajuste de modelos de memória longa, auto-regressivos, com quebra-estruturais ou ambos os casos. 


\section{Anexo}

Neste anexo vamos mostrar os códigos de programação dos softwares utilizados para ajustar os modelos no Capítulo 6.

Considere a série $y$ a ser analizada.

\subsection{Testes de Memória Longa}

Para realizar os testes de memória longa, usamos o software S-PLUS, com os seguintes códigos:

Load/Module/FinMetrics

$>\mathrm{y}=\mathrm{SDF} \$ \mathrm{~V} 1$

"Teste R/S"

$>\operatorname{rosTest}(\mathrm{y})$

"Teste de GPH"

$>$ gphTest(y) 


\subsection{Modelos ARFIMA (p,d,q)}

Para ajustar um modelo ARFI (p,d), no software S-PLUS, usamos os seguintes códigos:

Load/Module/FinMetrics

$>\mathrm{y}=\mathrm{SDF} \$ \mathrm{~V} 1$

"Escolha da ordem auto-regressiva do modelo, indo de 0 a 18"

$>$ far $<-F A R(y$, p.range $=c(0,8), C I=T)$

$>$ far\$BIC.all

"ARFI(2,d)"

$>\operatorname{arfi}<-F A R I M A(y, p=2, C I=T)$

$>$ arfi

"Diagnóstico"

$>\operatorname{arfi} \$ \mathrm{~m}$

$>$ arfi\$model

$>\operatorname{arfi} \$ \mathrm{cov}$

$>\operatorname{arfi} \$ \mathrm{BIC}$

$>$ arfi\$n.used

$>$ arfi\$loglike

>arfi\$residuals

Para ajustar um modelo ARFI (p,d), no software R, usamos os seguintes códigos:

$>\mathrm{y}<-$ ts $($ data $=$ usa, $\operatorname{start}=\mathrm{c}(1969,1)$, end $=\mathrm{c}(2004,6)$, frequency $=12$, deltat $=1 / 12)$

$>$ library(fracdiff)

"ARFI(2,d)" 
$>$ arfi<-fracdiff(y,nar=2)

\subsection{Modelo FI-STAR}

Para ajustar um modelo FI-STAR, no software R, usamos os seguintes códigos:

$>\mathrm{y}<-$ ts $($ data $=$ usa, $\operatorname{start}=c(1969,1)$, end $=c(2004,6)$, frequency $=12$, deltat $=1 / 12)$

$>$ library(fracdiff)

"ARFI(2,d)"

$>$ arfi<-fracdiff(y,nar=2)

"Diferença Fracionária"

$>$ z<-diffseries $(y, 0.4853336)$

$>\operatorname{library}(\mathrm{tsDyn})$

"FI-STAR com dois regimes e variável de transição $y_{t-2}, c=0.071847$ e gama=17.28"

$>$ mod.lstar $<-1 \operatorname{tar}(\mathrm{z}, \mathrm{mL}=2, \mathrm{mH}=2$, thDelay $=2, \mathrm{th}=0.071847$, gamma $=17.28$,

control=list $($ maxit $=3000)$ )

$>$ summary(mod.lstar)

"FI-SETAR com dois regimes e variável de transição $y_{t-2}$ "

$>$ mod.setar<-setar $(\mathrm{z}, \mathrm{mL}=2, \mathrm{mH}=2$, thDelay $=2)$

$>$ summary(mod.setar) 


\subsection{Algoritmo de Estimação Sequiencial dos Pontos de Quebras}

Estruturais

Para determinar os pontos de quebra estrutural usando o algoritmo de estimação seqüencial de Bai \& Perron (2003), no software R, usamos os seguintes códigos:

$>\mathrm{y}<-\mathrm{ts}($ data $=\mathrm{Bev}, \mathrm{start}=\mathrm{c}(1500), \mathrm{end}=\mathrm{c}(1845)$, frequency $=1$, deltat $=1)$

$>$ library(strucchange)

"Estimação seqüencial do número de quebras"

$>$ bp.y<-breakpoints $(y \sim 1)$

>summary(bp.y)

$>$ plot(bp.y)

"Gráfico com a série e as quebras estruturais"

$>$ plot(y,xlab="Tempo",ylab="Beveridge")

$>$ lines(bp.y)

$>$ fm $1<-\operatorname{lm}(\mathrm{y} \sim$ breakfactor(bp.y, breaks $=4))$

$>$ ts.fm1<-ts(data=fitted $(\mathrm{fm} 1), \operatorname{start}=\mathrm{c}(1500)$, end $=\mathrm{c}(1845)$, frequency $=1$, deltat $=1)$

$>$ lines(ts.fm1, col = 4)

\subsection{Modelo FI-BREAK}

Para ajustar um modelo FI-BREAK, no software RATS, usamos os seguintes códigos: 


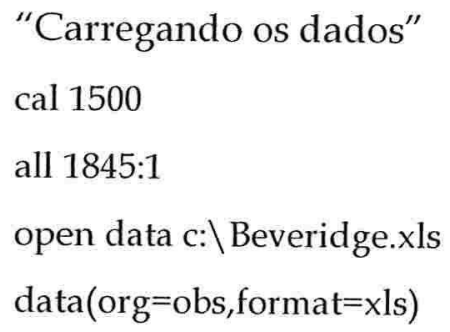




\section{Referências Bibliográficas}

Andersson, M. K., Eklund, B., Lyhagen, J. (1999). A simple linear time series model with misleading nonlinear properties. Economics Letters, 65, 281-284.

Andrews, D. W. K. (1991). Heteroscedasticity and autocorrelation consistent covariance matrix estimation. Econometrica, 59, 817-858.

Andrews, D. W. K. (1993). Tests for parameter instability and structural change with an unknown change point. Econometrica, 61, 821-856.

Andrews, D. W. K., Ploberger W. (1994). Optimal tests when a nuisance parameter is present only under the alternative. Econometrica, 62, 1383-1414.

Andrews, D. W. K., I. Lee, and W. Ploberger (1996). Optimal changepoint tests for normal linear regression. Journal of Econometrics, 70, 9-38.

Bai, J. S. and Perron, P. (1998). Estimating and testing linear models with multiple structural changes. Econometrica, 66, 47-78.

Bai, J. S. and Perron, P. (2003). Computation and analysis of multiple structural change models. Journal of Applied Econometrics, 18, 1-22. 
Baillie, R. T. (1996). Long memory processes and fractional integration in econometrics. Journal of Econometrics, 73, 5-59.

Bellman, R. and Roth, R. (1969). Curve fitting by segmented straight lines. Journal of the American Statistical Association, 64, 1079-1084.

Beran, J. (1995). Maximum likelihood estimation of the difference parameter for invertible short and long memory autoregressive integrated moving average models. Journal of the Royal Statistical Society, 57, 659-672.

Bos, C., Franses, P. H., Ooms, M. (1999). Long memory and level shifts: reanalyzing inflation rates. Empirical Economics, 24, 427-449.

Brown, R. L., Durbin J., Evans, J. M. (1975). Techniques for testing the constancy of regression relationships over time. Journal of the Royal Statistical Society, Series B 37, 149-192.

Chan, K. S. (1993). Consistency and limiting distribution of the least squares estimator of a threshold autoregressive model. Annals of Statistics, 21, 520-533.

Chen, R. (1995). Threshold variable selection in open-loop threshold autoregressive models. Journal of Time Series Analysis, 16, 461-481.

Chow, G. C. (1960). Tests of equality between sets of coefficients in two linear regressions. Econometrica, 28, 591-605.

Christiano, L. J. (1992). Searching for a break in GDP. Journal of Business and Economic Statistics, 10, 237-249. 
Davidson, J. (2000). When is a time series $\mathrm{I}(0)$ ? Evaluating the memory properties of nonlinear dynamic models. Unpublished manuscrit, Cardiff University.

de Gooijer, J. G. (1998). On threshold moving-average models. Journal of Time Series Analysis, 19, 1-18.

Diebold, F. X. and Inoue, A. (2001). Long memory and regime switching. Journal of Econometrics, 105, 131-159.

Eitrheim, Ø. and Teräsvirta, T. (1996). Testing the adequacy of smooth transition autoregressive models. Journal of Econometrics, 74, 59-76.

Enders, W. (1995). Applied Econometric Time Series. John Wiley \& Sons, Inc., USA.

Engle, R. F. and Smith, A. D. (1999). Stochastic permanent breaks. Review of Economics and Statistics, 81, 553-574.

Fisher, W. D. (1958). On grouping for maximum homogeneity. Journal of the American Statistical Association, 53, 789-798.

Fox, R. and Taqqu, M. S. (1986). Large sample properties of parameter estimates for strongly dependent stationary gaussian time series. The Annals of Statistics.

Franses, P. H. and van Dijk, D. (2000). Nonlinear Time Series Models in Empirical Finance. Cambridge: Cambridge University Press.

Gallant, A. R. (1987). Nonlinear Statistical Models. New York: John Wiley.

Geweke, J. and Porter-Hudak, S. (1983). The estimation and application of long memory time series models. Journal of Time Series Analysis, 4, 221-238. 
Granger, C. W. J (1993). Strategies for modelling nonlinear time-series relationships. The Economic Record, 69, 233-238.

Granger, C. W. J., Hyung, N. (2001). Occasional structural breaks and long memory. UCSD Working Paper.

Granger, C. W. J. and Joyeux, R. (1980). An introduction to long memory time series models and fractional differencing. Journal of Time Series Analysis, 1, 15-29.

Granger, C. W. J., Teräsvirta, T. (1999). A simple nonlinear time series model with misleading linear properties. Economic Letters, 62, 161-165.

Gupta, S. N. and Kumar, A. (1991). Time domain estimation in fractionally differenced noise processes. Journal of Combinatory Information and System Science.

Gupta, S. N. and Ji, X. (1992). Modeling persistence in time series data. Estadística.

Guthery, S. B. (1974). Partition regression. Journal of the American Statistical Association, 69, 945-947.

Hamilton, J. D. (1989). A new approach to the economic analysis of nonstationary time series and the business cycle. Econometrica, 57, 357-384.

Hamilton, J. D. (1994). Time Series Analysis. Princeton: Princeton University Press.

Hannan, E. J. (1973). The asymptotic theory of linear time series models. Journal of Applied Probability, 10, 130-145. 
Hansen, B. E. (1992). Testing for parameter instability in regressions with I(1) process. Journal of Business and Economic Statistics, 10, 321-335.

Hansen, B. E. (1996). Inference when a nuisance parameter is not present under the null hypothesis. Econometrica, 64, 413-430.

Hendry, D. F. (1995). Dynamic Econometrics. Oxford: Oxford University Press.

Hosking, J. R. M. (1981). Fractional differencing. Biometrika, 68, 165-176.

Hurst, H. E. (1951). Long-term storage capacity of reservoirs. Transactions of the American Society of Civil Engineers, 16, 770-799.

Hurst, H. E. (1957). A suggested statistical model of time series that occur in nature. Nature, 180, 494.

Hyung, N. and Franses, P. H. (2005). Forecasting time series with long memory and level shifts. Journal of Forecasting, 24, 1-16.

LeBaron, B. (1992). Some relationships between volatility and serial correlations in stock market returns. Journal of Business, 65, 199-219.

Leyborne, S., Newbold, P., Vougas, D. (1998). Unit roots and smooth transitions. Journal of Time Series Analysis, 19, 83-97.

Lo, A. W. (1991). Long term memory in stock market prices. Econometrica, 59, 12791313. 
Luukkonen, R., Saikkonen, P., Teräsvirta, T. (1988). Testing linearity against smooth transition autoregressive models. Biometrika, 75, 491-499.

Mandelbrot, B. B. and Van Ness, J. W. (1968). Fractional Brownian motion, fractional noises and applications. SIAM Review, 10, 422-437.

Morettin, P. A. e Toloi, C. M. C. (2006). Análise de Séries Temporais. $2^{\text {a }}$ Ed. São Paulo: Ed. Edgard Blücher Ltda.

Newey, W. K. and West, K. D. (1987). A simple positive semidefinite heteroskedasticity and autocorrelation consistent covariance matrix. Econometrica, $55,703-708$.

Quandt, R. (1983). Computational problems and methods. Handbook of Econometrics. Amsterdam: Elsevier Science, 699-746.

Reisen, V. A. (1994). Estimation of the fractional differenced parameter in the $\operatorname{ARIMA}(\mathrm{p}, \mathrm{d}, \mathrm{q})$ model using the smoothed periodogram. Journal of Time Series Analysis, 85, 338-344.

Sargan, J. D. (1964). Wages and prices in the United Kingdom: a study in econometric metodology. Econometric Analysis for National Economic Planning. Butterworths: London, 25-54.

Sin, C. Y. and White, H. (1996). Information criteria for selecting possibly misspecified parametric models. Journal of Econometrics, 71, 207-225. 
Skalin, J. (1998). Testing linearity against smooth transition autoregression using a parameter bootstrap. SSE/EFI Working Paper Series in Economics and Finance No 276, Stockholm School of Economics.

Teräsvirta, T. (1994). Specification, estimation, and evaluation of smooth transition autoregressive models. Journal of the American Statistical Association, 89, 208-18.

Teräsvirta, T. (1998). Modelling economic relationships with smooth transition regressions. Handbook of Applied Economic Statistics, Marcel Dekker, New York, A. Ulah, D.E.A. Giles (Ed.), pp. 507-552.

Tong, H. (1978). On a threshold model. Pattern Recognition and Signal Processing. Amsterdam, Sijthoff \& Noordhoff, 101-141.

Tong, H. (1983). Threshold models in non-linear time series analysis. Lecture Notes in Statistics, 21. Berlin: Springer.

Tong, H. (1990). Non-linear time series: a dynamical systems approach. Oxford, Oxford University Press.

van Dijk, D. J. C., Franses, P. H. (1999). Modelling multiple regimes in the business cycle. Macroeconomic Dynamics, 3, 311-340.

van Dijk, D. J. C., Franses, P. H., Paap, R. (2002). A nonlinear long memory model for US unemployment. Journal of Econometrics, 110, 135-165.

Vinod, H. D. (1991). Estimating fractionally integrated ARMA model persistence in GNP. Preprint. 
White, H. and Domowitz, I. (1984). Nonlinear regression with dependent observations. Econometrica, 52, 143-161.

Wooldridge, J. M. (1994). Estimation and inference for dependent processes. In: Engle, R.F., McFadden, D.I. (Eds.) Handbook of Econometrics, Vol.4, North-Holland, Amsterdam, pp. 2639-2738. 